Louisiana State University

LSU Digital Commons

Faculty Publications

Department of Physics \& Astronomy

7-21-2014

\title{
Quantum information with structured light
}

Mohammad Mirhosseini

California Institute of Technology

O. S. Magaña-Loaiza

University of Rochester Institute of Optics

M. N. O'Sullivan

University of Rochester Institute of Optics

B. Rodenburg

University of Rochester Institute of Optics

Z. Shi

University of South Florida, Tampa

See next page for additional authors

Follow this and additional works at: https://digitalcommons.Isu.edu/physics_astronomy_pubs

\section{Recommended Citation}

Mirhosseini, M., Magaña-Loaiza, O., O'Sullivan, M., Rodenburg, B., Shi, Z., Malik, M., Lavery, M., Padgett, M., Gauthier, D., \& Boyd, R. (2014). Quantum information with structured light. Optics InfoBase Conference Papers https://doi.org/10.1364/LS.2016.LTu1E.3

This Conference Proceeding is brought to you for free and open access by the Department of Physics \& Astronomy at LSU Digital Commons. It has been accepted for inclusion in Faculty Publications by an authorized administrator of LSU Digital Commons. For more information, please contact ir@lsu.edu. 


\section{Authors}

Mohammad Mirhosseini, O. S. Magaña-Loaiza, M. N. O'Sullivan, B. Rodenburg, Z. Shi, M. Malik, M. P.J. Lavery, M. J. Padgett, D. J. Gauthier, and Robert W. Boyd 


\title{
Quantum Information with Structured Light
}

\author{
by \\ Mohammad Mirhosseini
}

Submitted in Partial Fulfillment of

the Requirements for the Degree

Doctor of Philosophy

Supervised by

Professor Robert W. Boyd

The Institute of Optics

Arts, Sciences and Engineering

Edmund A. Hajim School of Engineering and Applied Sciences

\author{
University of Rochester \\ Rochester, New York
}


To my parents, Parvaneh and Kazem, and the love of my life, Vala. 


\section{Biographical Sketch}

Mohammad Mirhosseini was born in Tehran, Iran in 1987. After graduating from Allame Helli high school in 2005, Mohammad joined the University of Tehran where he double-majored in Electrical Engineering and Physics. Mohammad started the PhD program in Optics at the University of Rochester in 2010. Following the $\mathrm{PhD}$ qualifying exam, he joined the research group of Professor Robert W Boyd. Mohammad received his Master's degree in Optics in 2013.

\section{The following publications were a result of work conducted during doctoral study:}

[1] Mirhosseini, M., Magaña-Loaiza, O. S., Chen, C., Karimi, E., Hashemi Rafsanjani, S. M., Boyd, R. W. (2015). Wigner distribution of twisted photons. under review .

[2] Mirhosseini, M., Magaña-Loaiza, O. S., O’Sullivan, M. N., Rodenburg, B., Malik, M., Lavery, M. P. J., Padgett, M. J., Gauthier, D. J., Boyd, R. W. (2015). High-dimensional quantum cryptography with twisted light. New Journal of Physics, 17, 033033. 
[3] Mirhosseini, M., Magaña-Loaiza, O. S., Hashemi Rafsanjani, S.M., Boyd, R. W. (2014).Compressive Direct Measurement of the Quantum Wave Function. Physical Review Letters, 113, 090402.

[4] Mirhosseini, M., Malik, M., Shi, Z., Boyd, R. W. (2013). Efficient separation of orbital angular momentum eigenstates of light. Nature Communications, 4, 2781.

[5] Mirhosseini, M., Magaña-Loaiza, O. S., Chen, C., Rodenburg, B., Malik, M., Boyd, R. W. (2013). Rapid Generation of Light Beams Carrying Orbital Angular Momentum. Optics Express, Vol. 21, Issue 25, 3019630203.

[6] Mirhosseini, M., Rodenburg, B., Malik, M., Boyd, R. W. (2013). Freespace communication through turbulence: a comparison of plane-wave and orbital-angular-momentum encodings. Journal of Modern Optics, Vol. 61, Issue $1,43-48$.

[7] Shi, Z., Mirhosseini, M., Margiewicz, J., Malik, M., Rivera, F., Zhu, Z., Boyd, R. W. (2015). Scan-free direct measurement of an extremely highdimensional photonic state. Optica, 2 (4), 388-392.

[8] Malik, M., Mirhosseini, M., Lavery, M. P. J., Leach, J., Padgett, M. J., Boyd, R. W. (2014). Direct Measurement of a 27-Dimensional Orbital Angular Momentum State Vector. Nature Communications, 5, 3115.

[9] Rodenburg, B., Mirhosseini, M., Malik, M., Magaña-Loaiza, O. S., Yanakas, M., Maher, L., Steinhoff, N. K., Tyler, G. A., Boyd, R. W. (2014). Simu- 
lating thick atmospheric turbulence in the lab with application to orbital angular momentum communication. New Journal of Physics, 16, 033020.

[10] Rodenburg, B., Mirhosseini, M., Magaña-Loaiza, O. S., Boyd, R. W. (2014). Experimental generation of an optical field with arbitrary spatial coherence properties. JOSA B, Vol. 31, Issue 6, pp. A51-A55.

[11] Malik, M., O'Sullivan, M. N., Rodenburg, B., Mirhosseini, M., Leach, J., Lavery, M. P. J., Padgett, M. J., Boyd, R. W. (2012). Influence of atmospheric turbulence on optical communications using orbital angular momentum for encoding", Optics Express, Vol. 20, Issue 12, 13195-13200.

[12] Rodenburg, B., Lavery, M. P. J., Malik, M., O'Sullivan, M. N., Mirhosseini, M., Robertson, D. J., Padgett, M., Boyd, R. W. (2012). Influence of atmospheric turbulence on states of light carrying orbital angular momentum. Optics Letters, Vol. 37, Issue 17, 3735-3737.

[13] O'Sullivan, M. N., Mirhosseini, M., Malik, M., Boyd, R. W. (2012). Nearperfect sorting of orbital angular momentum and angular position states of light. Optics Express, Vol. 20, Issue 22, 24444-24449.

[14] Boyd, R. W., Rodenburg, B., Mirhosseini, M., Barnett, S. M. (2011). Influence of atmospheric turbulence on the propagation of quantum states of light using plane-wave encoding. Optics Express, Vol. 19, Issue 19, 18310-18317.

[15] Safari, A., De Leon, I., Mirhosseini, M., Magaña-Loaiza, Boyd, R. W. 
(2015). Light-Drag Enhancement by a Highly Dispersive Rubidium Vapor.Physical Review Letters accepted.

[16] Magaña-Loaiza, O. S., Mirhosseini, M., Cross, R. C., Hashemi Rafsanjani, S.M., Boyd, R. W. (2015). Hanbury Brown and Twiss Interferometry with Twisted Light. arXiv:1502.02086, under review.

[17] Potocek, V., Miatto, F. M., Mirhosseini, M., Magaña-Loaiza, O. S., Liapis, A. C., Oi, D. K. L., Boyd, R. W., Jeffers, J. (2015). The Quantum Hilbert Hotel. Physical Review Letters, 115, 160505.

[18] Hashemi Rafsanjani, S. Mirhosseini, M., Magaña-Loaiza, O. S., Boyd R. W. (2015). State transfer with classical non-separability. Phys. Rev. A, 92, 023827 .

[19] Gregg, P., Mirhosseini, M., Rubano, A., Marrucci, L., Karimi, E., Boyd, R. W., Ramachandran, S. (2015). Q-Plates as Higher Order Polarization Controllers for Orbital Angular Momentum Modes of Fiber. Optics Letters, 40 (8), 1729-1732.

[20] Magaña-Loaiza, O. S., Mirhosseini, M., Rodenburg, B., Boyd, R. W. (2014). Amplification of Angular Rotations Using Weak Measurements. Physical Review Letters, 112, 200401.

[21] Bouchard, F., Mand, H., Mirhosseini, M., Karimi, E., Boyd, R. W. (2014). Achromatic orbital angular momentum generator. New Journal of Physics, 16,123006 . 
[22] Nooramin, A., Mirhosseini, M., Shahabadi M. (2010). Modal analysis of multilayer conical dielectric waveguides for azimuthal invariant modes. Progress In Electromagnetics Research, Vol. 105, 213-229. 


\section{Acknowledgments}

First, I would like to express my special appreciation and thanks to my advisor, Professor Robert W Boyd. It has been truly a unique opportunity to work in your group, Bob. In working with you I have discovered the wonderful universe of experimental physics, and I have enjoyed interacting and collaborating with a large group of exceptionally talented scientists. I could not have imagined having a better advisor and mentor for my PhD study.

I would like to thank Professor Qiang Lin, Professor Nick Vamivakas, and Professor Andrew Jordan for agreeing to serve on my committee, and for the valuable discussions and guidance they have provided during the time I have worked on this thesis. I would like to thank Professor Miles Padgett and Professor Daniel Gauthier for the valuable research collaborations of their research groups. In addition, I thank many of the faculty with whom I have interacted and from whom I have learned all these past years, including Miguel Alonso, Lukas Novotny, Carlos Stroud, Joe Eberly, Jim Fienup, Steve Teitel, John Howell, Lynne Orr, and Peter Milonni.

During these years I have enjoyed and benefitted from the discussions with many colleagues and group mates including Mehul Malik, Brandon Rodenburg, 
Zhimin Shi, Malcolm O’Sullivan, Omar S. Magaña-Loaiza, Andreas Liapis, Martin Lavery, Joe Vornehm, Changchen Chen, Aizhong Zhang, Aaron Schweinsberg, Boshen Gao, Alex Radunsky, Heedeuk Shin, George Gehring, Petros Zerom, Gerardo Viza, Joe Choi, Mahmud Siddiqui, Robert Cross, and Jiapeng Zhao. Also, I would like to sincerely thank my friends Khashayar Kotobi, Navid Nakhjiri, Ashkan Pourkand, Omid Rajabi, Mohammad Kayedkhordeh, Ramtin Pedarsani, Kayvan Samimi, Mostafa Khoshnevisan, Hamid Ahmadi, Saeed Manaffam, Sahar Hashemgelgoroodi, and Mohammad Kazemi. I like to specifically thank my friends Seyed Mohammad Hashemi Rafsanjani and his wife Sara Nazari for their friendship and help, and for all the wonderful discussions that Mohammad and I have had together.

I would like to express my deepest appreciation to my parents and my sisters for their love and support, and for their sacrifice during my graduate school years when I have not been able to visit them. Finally I would like to express my appreciation to the love of my life, Vala, for her unconditional love and for always encouraging me to achieve the most. 


\section{Abstract}

Quantum information science promises dramatic progress in a variety of fields such as cryptography, computation, and metrology. Although the proof-ofprinciple attempts for implementing quantum protocols have often relied on only a few qubits, the utilization of more sophisticated quantum systems is required for practical applications. In this thesis, we investigate the emerging role of high-dimensional optical states as a resource for encoding quantum information.

We begin the first chapter with a review of orbital angular momentum (OAM) as a prime candidate for realizing multilevel quantum states and follow with a brief introduction to the quantum measurement theory. The second and the third chapters are dedicated to the application of OAM modes in quantum cryptography. In the second chapter, we discuss the challenges of projective measurement of OAM at the single-photon level, a crucial task required for quantum information processing. We then present our development of an efficient and accurate mode-sorting device that is capable of projectively measuring the orbital angular momentum of single photons. In the third chapter, we discuss the role of OAM modes in increasing the information capacity of quantum cryptography. We start this chapter by establishing the merits of encoding information on the 
quantum index of OAM modes in a free-space link. We then generalizing the BB-84 QKD protocol to the Hilbert space spanned by a finite number of OAM modes and outline our experimental realization.

The last two chapters are dedicated to the tomography of structured light fields. We start the fourth chapter by applying the recently found method of direct measurement to the characterization of OAM superpositions. We find the quantum state in the Hilbert space spanned by 27 OAM modes by performing a weak measurement of orbital angular momentum (OAM) followed by a strong measurement of azimuthal angle. We then introduce the concept of compressive direct measurement (CDM). In this method, we combine the direct measurement with an efficient computational technique known as compressive sensing in determining the wave function of an a priori unknown state. Using this combination, we demonstrate a 300-fold speed up in the measurement of a 19200-dimensional state. We demonstrate a technique for full characterization of the orbital-angular-momentum content of a photon in the fifth chapter. We achieve this task by directly measuring the elements of the density matrix in the basis of azimuthal angle, and subsequently mapping the results to the conjugate basis of OAM via a linear transformation. We provide data for experimental characterization of pure OAM superpositions as well as mixed combinations of OAM modes using this technique. 


\section{Contributors and Funding}

\section{Sources}

The thesis committee examining this dissertation is comprised of Professor Robert W. Boyd and Professor Nick Vamivakas from the Institute of Optics, and Professor Qiang Lin from the Electrical Engineering department at the University of Rochester. The committee is chaired by Professor Andrew N. Jordan from the Physics department at the University of Rochester. The research performed during the course of this thesis is a result of many fruitful collaborations within my research group as well as outside of it.

Chapter one provides the necessary background material for this thesis and contains no original research.

The content of chapter two is primarily adapted from peer-reviewed publications in the journals Nature Communications [1] and Optics Express [2]. The work in [1] was led by Mohammad Mirhosseini, and the work in [2] was led by Malcolm N. O'Sullivan. The research was carried out in the laboratory of Professor Boyd with contributions from Mehul Malik and Zhimin Shi. The background information in section two is based on previous results of other workers [3, 4]. 
This research was supported by the DARPA InPho program led by Professor Dan Gauthier of Duke University

The content of chapter three is primarily adapted from peer-reviewed publications in New Journal of Physics [5], Optics Express [6], and Journal of Modern Optics [7]. The work in all three references was led by Mohammad Mirhosseini. The research was primarily conducted in the laboratory of Professor Boyd with contributions from Malcolm N. O'Sullivan, Mehul Malik, Brandon Rodenburg, Omar S. Magaña-Loaiza, and Changchen Chen. This project has been part of a collaboration with Martin P. J. Lavery, Professor Miles J Padgett, and Professor Daniel J. Gauthier. This research was supported by the DARPA InPho program.

The content of chapter four is primarily adapted from peer-reviewed publications in Physical Review Letters [8] and Nature Communications [9]. The work in [8] was led by Mohammad Mirhosseini, and the work in [9] was led by Mehul Malik. The work presented in [8] was conducted in the laboratory of Professor Boyd with contributions from Omar S. Magaña-Loaiza and Seyed Mohammad Hashemi Rafsanjani. The work presented in [9] was conducted in the laboratory of Professor Boyd in collaboration with Martin P. J. Lavery, Jonathan Leach, and Professor Miles J Padgett. The background information in section two is based on previous results of other workers [10]. This research was supported by the DARPA InPho program.

The content of chapter five is from a research project led by Mohammad Mirhosseini. The manuscript containing the results is under preparation. The research pertaining to this chapter was conducted in the laboratory of Profes- 
sor Boyd with contributions from Omar S. Magaña-Loaiza Seyed Mohammad Hashemi Rafsanjani, and Changchen Chen. This research was supported by an ONR grant. 


\section{Table of Contents}

Biographical Sketch . . . . . . . . . . . . . . . . iii

Acknowledgments . . . . . . . . . . . . . . viii

Abstract . . . . . . . . . . . . . . . $x$

Contributors and Funding Sources . . . . . . . . . . . . . xii

List of Figures . . . . . . . . . . . . . . . . . . xvi

1 Key concepts 1

1.1 Orbital angular momentum of the light field . . . . . . . . . . . 1

1.2 Quantum measurements ... . . . . . . . . . 6

1.2.1 Projective measurements . . . . . . . . . . . . . 6

1.2.2 Weak measurements ............. . 8

2 Measurement of orbital angular momentum 12

2.1 Introduction . . . . . . . . . . . . . . . . . . . . . . . . . 12

2.2 Log-polar coordinate transformation . . . . . . . . . . . . . . . 14

2.3 Enhanced sorting via refractive beam copying . . . . . . . . . . 17

2.4 Characterization of the mode sorter for application in QKD . . . 27

2.5 Summary ...................... 32

3 Quantum cryptography with twisted photons 33

3.1 Introduction . . . . . . . . . . . . . . . . 33

3.2 Free-space communication with spatial modes . . . . . . . . . . 35

3.3 BB-84 QKD with OAM modes . . . . . . . . . . . . . . 42

3.4 Generation of OAM and ANG modes . . . . . . . . . . . . . 48

3.5 Experimental realization . . . . . . . . . . . . 54 
3.6 Steps towards practical QKD with OAM modes . . . . . . . . 66

3.7 Summary . . . . . . . . . . . . . . . . . . . . 69

4 Direct measurement of the wave function $\quad 70$

4.1 Introduction . . . . . . . . . . . . . . . . . . . . 70

4.2 Theory and background . . . . . . . . . . . . . . . 72

4.3 Direct measurement of the OAM state vector . . . . . . . . . 75

4.4 Compressive direct measurement . . . . . . . . . . . . . . . 83

4.5 Summary ........................ 92

5 Characterization of mixed OAM states 93

5.1 Introduction . . . . . . . . . . . . . . . . . . . . . 93

5.2 Measurement of the density matrix . . . . . . . . . . . . . . . 95

5.3 Experimental realization . . . . . . . . . . . . . . . . . 97

5.4 Summary . . . . . . . . . . . . . . . . . . 103

$\begin{array}{ll}\text { Conclusions } & 104\end{array}$

$\begin{array}{ll}\text { Appendix A: Diffraction and radial modes } & 107\end{array}$

Appendix B: Data for characterization of mixed OAM states 111

$\begin{array}{lr}\text { Bibliography } & 114\end{array}$ 


\section{List of Figures}

1.1 a) The polarization vector of a circularly polarized beam carrying SAM. b)The wavefront of a beam carrying OAM. . . . . . . . . 3

2.1 The image of the OAM mode sorter device from Ref. [4]. (a) The optical transformation converts the OAM modes to plane waves as they go through the first refractive phase element. The second refractive elements corrects the phase distortion. (b) An SLM is used to realize an $\ell$-forked hologram. A 4 f system is used along with a spatial filter to generate OAM modes. The sorter elements reshape the mode and the lens focuses the separated OAM modes onto a CCD camera. . . . . . . . . . . . . . . 16

2.2 Simulated results demonstrating the transformation of the modes undergoing the log-polar coordinate transformation. . . . . . . . 16 
2.3 Effect of beam copying on mode overlap. (a) Two tilted plane waves resulting from the transformation of two neighboring OAM modes are focused to overlapping spots by a lens. (b) Magnified plane waves can be focused to narrower spots that are spaced closer to each other albeit with no change in overlap. (c) Multiple coherent copies of the transformed plane waves are placed next to each other resulting in focused spots with reduced overlap. (d) Experimental data for OAM modes focused after the log-polar mapping. The data for seven modes $\ell=-3: 3$ are super-imposed on top of each other. The $\ell=-2$ mode is displayed in false color to mark the overlap with the neighboring modes. (e) Experimental data for the modes from part $d$ when enhanced by making seven copies in the Fourier domain. Results are from Ref. [1]. . . . . . . . . . . . . . . . . . . . 23

2.4 Phase profiles for diffractive beam copying. (a) The phase grating for creating seven copies (shown on the left) along with the corresponding phase profile of the phase-correcting element (shown on the right). (b) The fan-out and phase corrector phase profiles for creating nine copies. . . . . . . . . . . . . . . 24

2.5 The schematic of the mode sorting set-up from Ref. [1]. The optical transformation converts the OAM modes to plane waves as they go through the first and second refractive phase elements. An SLM is used to create multiple copies of the unwrapped beam. A lens focuses the resulting wide beam into a spot, after the phase distortions are removed by the second SLM. (To simplify the demonstration, a fan-out element creating only three copies has been displayed. For further simplification, we have also eliminated the Fourier transform lens between the fanout and the phase corrector element.) . . . . . . . . . . . . . 25

2.6 Generation of OAM and ANG modes. Examples of holograms (top line) for generating OAM and ANG modes along with the intensity profile (bottom line) of the resulting spatial modes. . 25 
2.7 Intensity profile of the transformed OAM modes. The incident power in each OAM mode (summed along the vertical axis) as a function of the horizontal coordinate on the CCD camera. The center of each mode is labelled with the corresponding OAM mode index. The sum of the total power in each mode is normalized to unity. . . . . . . . . . . . . . . . . . . . 26

2.8 Intensity profile of the transformed ANG modes. The total incident power in each ANG mode as a function of the horizontal coordinate on the CCD camera. The center of each mode is labelled with the corresponding ANG mode index. Sum of the total power in each mode is normalized to unity. . . . . . . . . . 26

2.9 The conditional probability of detection. The horizontal axes indicate the indices for the sent and received (a) OAM modes and (b) ANG modes. . . . . . . . . . . . . . . . . . . . . . . . . 28

2.10 Mutual information as anction of the number of the modes. Mutual information as calculated for a) ideally separated modes, b) data from this experiment, c) perfect log-polar mapping, and d) perfect log-polar mapping combined with beam copying. All values are in bits per detected photons . . . . . . . . 29

3.1 Schematic diagram of a free-space communication link. . . . . . . 36

3.2 Information encoding by the location of distinct spots focused within the receiver's aperture. . . . . . . . . . . . . . . 38

3.3 Numerical results for the required aperture diameter as a function of the maximum OAM index . . . . . . . . . . . . . . . 40

3.4 Left panel: Table shows the MUBs used by Alice for encoding information. Each basis is made from two orthogonal quantum states. Right panel: Table demonstrates a sequence of random numbers sent by Alice and measured by Bob. Notice the perfect correlation of Alice and Bob's sequences when they have used the identical bases. Bob's sequence is statistically independent of that of Alice on the instances when the bases are different. . . . . . . . 43 
3.5 Channel capacity and error bound calculated for multiple values of $d$, for the case of $M=2$. The error bound values are quoted

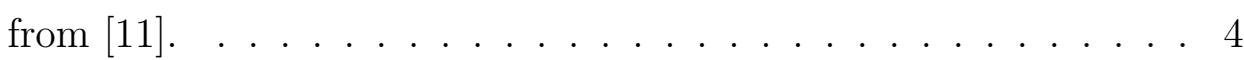

3.6 The experimentally measured intensity profile of OAM and ANG modes for the case of $d=7$. . . . . . . . . . . . . . . . . . 46

3.7 Binary hologram for generating (a) $\ell=2$ vortex OAM mode, (b) $\ell=-5$ vortex OAM mode, (c) $L G_{22}$ and (d) $L G_{21} \ldots$. . . . . . 49

3.8 Schematic diagram of the experimental setup for a) measuring intensity profile and phase interferograms. b) switching among three OAM modes and detecting them in real time. . . . . . . . . 50

3.9 Intensity and interferograms of three vortex modes (to the left) and LG modes (to the right). The interferograms demonstrate the phase structure of the beams and have been obtained by interfering the generated modes with a plane wave. . . . . . . . . 51

3.10 Intensity patterns for ANG modes constructed from superposition of vortex OAM modes from the set $\ell \in[-2: 2]$. . . . . . . . . 52

3.11 Detected power as function of time for three vortex OAM modes of $\ell=5, \ell=-5$ and $\ell=0$. It is seen that the generated mode can be changed on a time-scale of $0.25 \mathrm{~ms}$ (measured at FWHM) corresponding to a switching rate of $4 \mathrm{kHz}$. . . . . . . . . . . 53

3.12 a) The experimental setup. Alice prepares the modes by carving out pulses from a highly attenuated He-Ne laser using an AOM. The spatial mode information is impressed on these pulses with a DMD. Bob's mode sorter and fan-out elements map the OAM modes and the ANG modes into separated spots that are collected by an array of fibers. b) The alphabet. CCD images of produced light field profiles in two complementary spatial bases of OAM and ANG $(\mathrm{d}=7)$. The intensity profile of the modes are shown on the right. An example of binary holograms used for the generation of modes in each basis is shown on the left. . . . . . . 55 
3.13 Key generation a) An example of a random sifted key from the experiment. The spatial modes are mapped to number between 0 to 6 (errors are marked in red and underlined). Each symbol is converted into a 3 digit binary number first and the binary key is randomized before the error-correction. Privacy amplification minimizes Eve's information by shortening the key length. b) Alice encrypts the secret message (a picture [?]) using the shared secure key and Bob decrypts it. In this case a short key is repeated many times to match the bit length of the image. In practice, multiple use of a short key cannot provide security and a long key needs to be used. . . . . . . . . . . . . . . . . . . . . . . 56

3.14 Conditional probability of detection. a) Theoretical predictions for an ideal system. The bases chosen by Alice and Bob are marked on the horizontal axis. b) The experimental results. To construct the matrix a total number of 14 million pulses with $\mu=0.1$ photon per each are sent by Alice. The signals from APDs and the gate are collected using a $300 \mathrm{MHz}$ oscilloscope on the fast acquisition mode and processed on a computer. c) 3D view of the experimental data. . . . . . . . . . . . . . . . . . . 58

3.15 Error-bound for security. Bob's error bound calculated from theory as a function of the dimension of the Hilbert space for intercept-resend attacks with two MUBs $(\mathrm{M}=2)$ and coherent attacks is plotted along with symbol error rate measured from our experimental data (the error bar shows one standard deviation). It is seen that the symbol error rate from our experiment lies well below the theoretical bounds and hence it is sufficient for proof of security. . . . . . . . . . . . . . . . . 62

4.1 Left panel: Experimental setup for direct measurement. Right panel: a small polarization rotation can be pictured as the superposition of the original polarization plus a weak orthogonally polarized component. . . . . . . . . . . . . . . 73 
4.2 Experimental setup for direct measurement of a high-dimensional state vector. State Preparation: A quantum state in an arbitrary superposition of orbital-angular-momentum (OAM) modes is prepared by impressing phase information with a spatial light modulator (SLM1) onto spatially-filtered (SMF) photons from an attenuated HeNe laser. Weak Measurement: A particular OAM mode is weakly projected by rotating its polarization. In order to do so, the OAM modes are first transformed into finite-sized momentum modes by two refractive optical elements made out of PMMA (R1 and R2). Then, a Fourier transform lens (L1) and a fan-out hologram implemented on SLM2 are used to generate three adjacent copies of each momentum mode. The phase between these copies is corrected by SLM3. Another lens (L2) converts these larger momentum modes into well separated position modes at its focus. Finally, a quarter-wave plate (QWP0) used in double-pass with SLM4 are used to rotate the polarization of the OAM mode to be weakly projected. Another quarter-wave and half-wave plate (QWP1 and HWP1) are used to remove any ellipticity introduced by transmission and reflection through the non-polarizing beam splitter (NPBS). Strong measurement: A strong measurement of angular position is performed by Fourier transforming with a lens (L3) and post-selecting state $p=0$ with a $10 \mu \mathrm{m}$ slit. Readout: The OAM weak value $\left\langle\pi_{\ell}\right\rangle_{\mathrm{w}}$ is obtained by measuring the change in the photon polarization in the linear and circular polarization bases. QWP2, HWP2, a polarizing beam splitter (PBS), and two single-photon avalanche detectors (SPADs) are used for this purpose. . . . . . . . . . . . . . . 77 
4.3 Experimental data showing direct measurement of a 27-dimensional state vector in the OAM basis. The state is created by sending photons through an angular aperture of width $\Delta \theta=2 \pi / 9 \mathrm{rad}$ (inset of (b)). (a) The measured real (blue circles) and imaginary parts (red triangles) of the state vector, (b) the calculated probability density $|\Psi(\ell)|^{2}$, and (c) the calculated phase $\phi(\ell)$ are plotted as functions of the OAM quantum number $\ell$ up to a dimensionality of $\ell= \pm 13$. The probability density has a sinc-squared shape. The phase has an asymmetric quadratic shape due to small misalignments in our optical system. Additionally, $\pi$-phase jumps are seen in the phase when the probability amplitude changes sign (not seen in the probability density). Theoretical fits to the probability density and phase are plotted as blue lines. Error bars are calculated by propagating the detector error (due to background light and dark counts) through to all measured quantities. Error bars larger than the symbols are shown. The data shown is the average result obtained from 50 experimental runs. . . . . . . . . 79

4.4 Experimental data showing the direct measurement of a rotated high-dimensional state vector. The created state is rotated by angles $\theta_{ \pm}= \pm \pi / 9 \mathrm{rad}$ (insets of (b) and (e)). (a) and (d) The measured real (blue circles) and imaginary parts (red triangles) of the rotated state vectors. (b) and (e) The calculated probability densities $\left|\Psi(\ell)_{ \pm}\right|^{2}$. (c) and (f) The phase difference $\Delta \phi_{ \pm}(\ell)$ between the calculated phase and the phase of the unrotated case from Fig. ??(c). Theoretical fits to the probability densities and phases are plotted as blue lines. The linear fits in (c) and (f) are calculated via the process of chi-square minimization, which takes into account the error at each point. Error bars are calculated by propagating the detector error (due to background light and dark counts) through to all measured quantities. Error bars larger than the symbols are shown. The data shown is the average result obtained from 50 experimental runs. . . . . . . . . . . . 81 
4.5 A schematic illustration of the experimental setup. Collimated Gaussian beam from the single mode fiber is passed through a polarizer to prepare a vertical polarization. The SLM is used along with two quarter wave plates (WP1 and WP2) to rotate the polarization at each pixel. An $f=50 \mathrm{~cm}$ lens focuses the beam onto a pinhole with a diameter of $10 \mu \mathrm{m}$. The polarization measurement is performed on the light collected from the pinhole using a QWP/HWP (WP3) and a polarizing beam splitter. . . . . 86

4.6 The amplitude, real, and imaginary parts of an aberrated Gaussian state from experimental data. The left column presents data from a pixel-by-pixel scan of the state for $\mathrm{N}=192$. The middle column shows the reconstructed wavefront for for $\mathrm{N}=192$, and $M / N=20 \%$ of total measurements from the CDM method. The right column demonstrates reconstruction $\mathrm{N}=19200$, and $M / N=$ $20 \%$ of total measurements. The transverse dimensions of the state are shown in millimeters. . . . . . . . . . . . . . 87

4.7 The fidelity of the reconstructed state with the target wave function as a function of the percentage of the total measurements. The fidelity of the state reconstructed with CDM is shown in blue. The fidelity of the state reconstructed from a partial pixelby-pixel scan with the same number of measurements is shown in red for comparison. The error-bars represent standard deviation calculated from 100 repetitions of the experiment (error-bars are shown at every second data point for visual clarity). . . . . . . . . 89

4.8 The amplitude and phase of a Gaussian mode illuminating a custom phase mask (the initials of the University of Rochester). The data is reconstructed by the CDM method with $\mathrm{N}=19200$, and $M / N=20 \%$ of total measurements. . . . . . . . . . . . . 91 


\subsection{Characterization of the transverse structure of classical}

light. Top panel: The light beam from a HeNe laser illuminates a phase-only spatial light modulator. The polarization state of the beam is prepared by a polarizer. A Dove prism located inside a Sagnac interferometer causes a rotation in opposite directions of each of the counter-propagating beams. Two quarter wave plates along with a half wave is used along with a polarizing beam splitter for characterizing the polarization of the output beam. Bottom panel: Experimental results for characterization of an OAM mode with $\ell=1$. The top plot shows the density matrix in the (discretized) basis of azimuthal angle and the plot in the bottom is the measured density matrix in the OAM basis. . . . . 98

\subsection{Characterization of pure and mixed superposition states.}

Top panel: The intensity pattern of a pure (left) and mixed (right) superposition of $\ell=1$ and $\ell=-1$ OAM modes with equal weights. Bottom panel: The real and imaginary parts of the OAM density matrix from experiment. . . . . . . . . . . . . 100

5.3 Characterization of the transverse structure of single photons. Top left: A PPKTP crystal is pumped with a $405 \mathrm{~nm}$ continuous wave laser beam. Single photons from non-degenerate parametric down-conversion are separated with a dichroic mirror. The idler photons $(830 \mathrm{~nm})$ are detected by an avalanche photodiode (APD), which heralds the detection of signal photons (790 $\mathrm{nm}$ ) with an intensified charge coupled device (ICCD). A q-plate $(\mathrm{q}=1 / 2)$ is placed between two crossed polarizer to prepare an equal superposition of $\ell=1$ and $\ell=-1$ OAM modes. Top right: The transverse structure of single photons captured with an accumulation of 5-ns-coincidence events over a $1200 \mathrm{sec}$ exposure time. Bottom panel: The real and imaginary parts of the OAM density matrix from experiment. . . . . . . . . . . . . . . 102

4 Bob's and Eve's receiving apertures f . . . . . . 108 
5 Characterization of a coherent superposition and a mixture of angular wedges. Top panel: Elements of the density matrix for a coherent superposition of three angular wedges $\left(|\Psi\rangle=\left|\Theta_{1}\right\rangle+\left|\Theta_{3}\right\rangle+\left|\Theta_{5}\right\rangle\right)$. Bottom panel: Elements of the density matrix for an incoherent mixture of the same angular wedge states.111

6 Characterization of OAM eigenstates. Top panel: Elements of the density matrix for $\ell=0$. Middle panel: Elements of the density matrix for $\ell=1$. Bottom panel: Elements of the density matrix for $\ell=2$. . . . . . . . . . . . . . . . . . . . . . . 112

7 Characterization of a coherent superposition and a mixture of OAM eigenstates. Top panel: Elements of the density matrix for a coherent superposition of two OAM states $(|\Psi\rangle=$ $|\ell\rangle+|-\ell\rangle$, where $\ell=1)$. Bottom panel: Elements of the density matrix for an incoherent mixture of two OAM states $(\rho=$ $|\ell\rangle\langle\ell|+|-\ell\rangle\langle-\ell|$, where $\ell=1)$. . . . . . . . . . . . . 112

8 Characterization of a coherent superposition of OAM eigenstates imposed on the transverse structure of single pho-

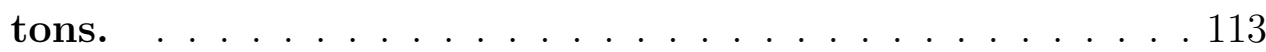




\section{Chapter 1}

\section{Key concepts}

\subsection{Orbital angular momentum of the light field}

Spin angular momentum of light has been a topic of theoretical research since the beginning of 20th century [12]. In 1936 it was experimentally verified that circularly polarized optical beams carry angular momentum [13]. The orbital angular momentum of light is perhaps a less familiar concept. In 1992 Allen et al. showed that a set of solutions to the paraxial Helmholtz equation known as Laguerre-Gaussian (LG) beams possess orbital angular momentum (OAM) [14]. This property is closely linked to the vortex phase structure of the LG beams, much similar to the quantum mechanical wavefunction of material particles with orbital angular momentum. The concept and theory of optical OAM as well as the applications of it has been an area of active research in the last twenty years.

In the classical theory of electromagnetism, the Poynting vector is used to calculate the energy and the linear momentum carried by optical fields. In this framework, the exchange of momentum between the fields and the charged particles can described using Maxwell's stress tensor. By extending the mechanical analogy to material particles, one might guess that electromagnetic fields carry angular momentum as well. This is in fact true, and that the total angular momentum of the electromagnetic field in vacuum can be defined as [15] 


$$
\begin{aligned}
\mathbf{J} & =\varepsilon_{0} \int \mathbf{r} \times[\mathbf{E}(\mathbf{r}, t) \times \mathbf{B}(\mathbf{r}, t)] d^{3} r \\
& =\int \mathbf{r} \times \mathcal{P} d^{3} r
\end{aligned}
$$

Here, $\mathcal{P}$ is the linear momentum density, and $\mathbf{J}$ is the total angular momentum of the electromagnetic fields. Note that we are treating the components of the fields as complex numbers, as it is common in the classical theory of electromagnetism. The transition to the quantum mechanical counterparts can be formally achieved by replacing the complex numbers with operators and performing the symmetrization procedure, wherever necessary [16].

In analogy to the non-relativistic quantum mechanics of material particles, it is desirable to separate the total angular momentum into its orbital and spin parts. Starting from the expression for $\mathbf{J}$ above, it is straightforward to show that $[17]$

$$
\mathbf{J}=\varepsilon_{0} \int d^{3} r\left\{\sum_{a} E_{a}(\mathbf{r} \times \nabla) A_{a}+\mathbf{E} \times \mathbf{A}\right\} .
$$

Where $\mathbf{A}$ is the transverse vector potential, defined by the following relations

$$
\begin{aligned}
& \nabla \times \mathbf{A}=\mathbf{B}, \\
& \nabla \cdot \mathbf{A}=0 .
\end{aligned}
$$

The two terms in Eq. (1.1.2) can be identified as [17]

$$
\begin{aligned}
& \mathbf{L}=\varepsilon_{0} \int d^{3} r \sum_{a} E_{a}(\mathbf{r} \times \nabla) A_{a} \\
& \mathbf{S}=\varepsilon_{0} \int d^{3} r \mathbf{E} \times \mathbf{A} .
\end{aligned}
$$

Note that all the electromagnetic fields $\mathbf{E}$ and $\mathbf{B}$ are assumed to be transverse fields to come up with this result (An arbitrary vector field $\mathcal{F}(\mathbf{r})$ can be decomposed to two transverse and longitudinal components where $\nabla \cdot \mathcal{F}_{\text {trans. }}(\mathbf{r})=0$ and $\nabla \times \mathcal{F}_{\text {longit. }}(\mathbf{r})=0$. For this reason, this decomposition is only valid for 
free-space electromagnetic fields in absence of sources of radiation [18, 17, 19]. The contribution of longitudinal fields in the linear and angular momentum is usually taken into account by modifying the Hamiltonian of the charged particles $[17,19]$. The difficulties associated with decomposing angular momentum to orbital and spin parts can be understood considering the fact that the spin of a particle represents its total angular momentum in the frame where it is at rest, and such a frame cannot be defined for particles with zero rest mass such as photons [17].

a)

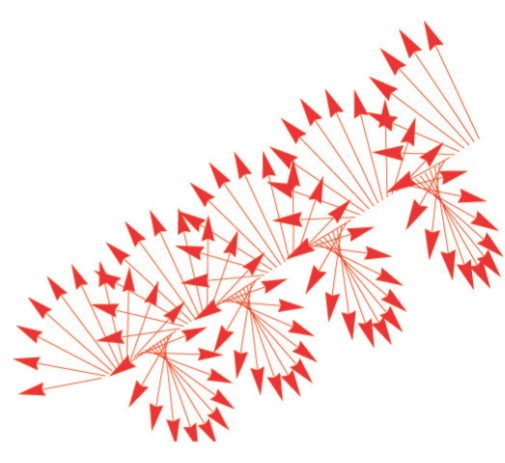

b)

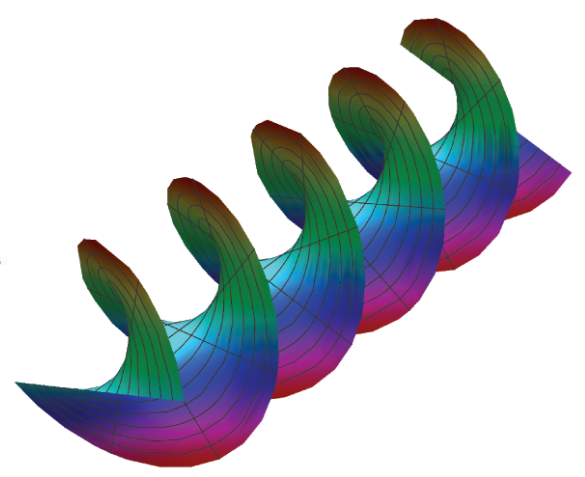

Figure 1.1: a) The polarization vector of a circularly polarized beam carrying SAM. b)The wavefront of a beam carrying OAM.

Nevertheless, it can be shown that within the paraxial approximation, the spin angular momentum (SAM) can be associated with polarization, and the orbital angular momentum (OAM) is related to the transverse spatial structure of the optical field (See Fig. (1.1)) [14]. We consider a polarized, circularly symmetric, paraxial beam represented by a vector potential of the form

$$
\mathbf{A}=(a \hat{\mathbf{x}}+b \hat{\mathbf{y}}) u(\rho, z) e^{-i \ell \phi} e^{i k z}
$$

Here, $(a \hat{\mathbf{x}}+b \hat{\mathbf{y}})$ denotes the polarization state, $u(\rho, z)$ describes the spatial profile of the beam, and $\rho=\sqrt{x^{2}+y^{2}}$. In this case, the cycle-averaged momentum 
density can be derived to be

$$
\begin{aligned}
\langle\mathcal{P}\rangle & =\frac{\varepsilon_{0}}{2}\left(\mathbf{E}^{*} \times \mathbf{B}+\mathbf{E} \times \mathbf{B}^{*}\right) \\
& =\omega \varepsilon_{0}\left(\left[i u \frac{\partial}{\partial \rho} u^{*}+\text { c.c. }\right] \hat{\boldsymbol{\rho}}+\left[\frac{|u|^{2}}{\rho} \ell-\frac{\sigma}{2} \frac{\partial|u|^{2}}{\partial \rho}\right] \hat{\boldsymbol{\phi}}+k|u|^{2} \hat{\mathbf{z}}\right) .
\end{aligned}
$$

Here, $\sigma=i\left(a b^{*}-b a^{*}\right)$ is a quantity that sets the helicity of polarization $(\sigma= \pm 1$ for circularly polarized light and $\sigma=0$ for linearly polarized light). In deriving the expression above we have dropped the terms that are proportional to $\frac{\partial u}{\partial z}$, in accordance with the paraxial approximation. Using the expression for $\langle\mathcal{P}\rangle$, the cycle-averaged angular momentum density along the optical axis, $\left\langle\mathcal{J}_{z}\right\rangle$ becomes

$$
\left\langle\mathcal{J}_{z}\right\rangle=\left\langle\mathcal{L}_{z}\right\rangle+\left\langle\mathcal{S}_{z}\right\rangle=\varepsilon_{0} \omega|u|^{2} \ell-\frac{\varepsilon_{0} \omega}{2} \rho \frac{\partial|u|^{2}}{\partial \rho} \sigma .
$$

The total value of angular momentum flux, $\left\langle J_{z}\right\rangle$, going through any plane normal to the optical axis can be calculated by integrating the angular momentum flux density $\left\langle\mathcal{J}_{z}\right\rangle$, and then multiplying it by $c$. To find the angular momentum for a single photon, we initially evaluate the ratio of angular momentum flux divided by the energy flux, $\left\langle\Phi_{z}\right\rangle$, of a classical beam of light

$$
\begin{aligned}
\frac{\left\langle J_{z}\right\rangle}{\left\langle\Phi_{z}\right\rangle}=\frac{\iint\left\langle\mathcal{J}_{z}\right\rangle \rho d \rho d \phi}{\iint c\left\langle\mathcal{P}_{z}\right\rangle \rho d \rho d \phi} & =\frac{c \varepsilon_{0} \omega \iint\left(|u|^{2} \ell-\frac{\rho}{2} \frac{\partial|u|^{2}}{\partial \rho} \sigma\right) \rho d \rho d \phi}{c \varepsilon_{0} \omega^{2} \iint|u|^{2} \rho d \rho d \phi} \\
& =\frac{\ell+\sigma}{\omega} .
\end{aligned}
$$

The extension to the quantum mechanical case can be simply done considering the energy of a single photon prepared in the mode described by Eq. (1.1.7) is equal to $\hbar \omega$. Consequently the total angular momentum for a single photon in this mode is found as

$$
J_{z}=L_{z}+S_{z}=\hbar \ell+\hbar \sigma
$$

Thus, we have shown that any cylindrically symmetric beam with a helical phase 
structure of $e^{-i \ell \phi}$ carries $\ell \hbar$ units of OAM. The set of LG modes are a famous example of such modes with that are often mentioned in optics literature[14]. In addition to the LG modes, the set of vortex modes defined by top-hat intensity structure and $e^{-i \ell \phi}$ phase profile are often studied due to their simplicity of experimental generation with a q-plate [20] or a phase plate [21]. 


\subsection{Quantum measurements}

\subsubsection{Projective measurements}

Measurement lies at the heart of quantum mechanics. The significance of measurement, as a theoretical concept, is reflected in a postulate of quantum mechanics, which is entirely devoted to defining the act of measurement. Besides the fundamental significance, a better understanding of quantum measurements has greatly contributed to progress in experimental physics. The best example of such progress is, perhaps, manifested in the evolution of the field of weak measurements since its introduction in 1988 by Aharonov, Albert, and Vaidman (AAV) [22]. In this section we provide a brief review of the mathematical formalism used to describe conventional measurements often encountered in quantum mechanics (i.e. projective measurements). Subsequently, we will review a generalization of this framework, which we will use for describing weak measurements.

A conventional quantum measurement can be described using a projection operator. The von Neumann interaction is a mathematical model for describing such measurements. In this model, the act of measurement is described as a coupling between a measured system and another variable that represents the measurement apparatus, often called as a pointer. The Hamiltonian for the interaction of an observable $\hat{A}$ of the quantum system coupled with the momentum $\hat{P}$ of the pointer can be written as

$$
\hat{\mathcal{H}}=g \hat{A} \otimes \hat{P},
$$

where $g$ is a coupling constant. For an interaction time of length $T$, the resulting time evolution operator is

$$
\hat{U}(T)=\exp \left(\frac{-i g T \hat{A} \otimes \hat{P}}{\hbar}\right) .
$$

We choose to work in the basis spanned by the eigenstates of $\hat{A}$ in order to evaluate the effect of $\hat{U}$ on the joint system-pointer quantum state. We note 
that $\hat{A}$ is diagonal in this basis.

$$
\hat{A}=\sum_{a}|a\rangle a\langle a|
$$

We assume an unentangled initial system-pointer quantum state $|\psi(0)\rangle=|I\rangle|\phi\rangle$. Here, the initial state of the quantum system, $|I\rangle$, can be written as a superposition of $\hat{A}$ eigenstates

$$
|I\rangle=\sum_{a} \alpha_{a}|a\rangle
$$

The effect of the time evolution operator on the joint system-pointer quantum system is thus found as

$$
\hat{U}(T)|\psi(0)\rangle=\hat{U}(T)\left(\sum_{a} \alpha_{a}|a\rangle \otimes|\phi(x)\rangle\right) .
$$

Note that $\hat{P}$ is the generator of translations for of the pointer, and hence

$$
e^{-i x_{0} \hat{P}} \phi(x)=e^{-i x_{0} \frac{d}{d x}} \phi(x)=\phi\left(x-x_{0}\right) .
$$

Subsequently, the state of the pointer and the system after the evolution becomes.

$$
\hat{U}(T)|\psi(0)\rangle=\sum_{a} \alpha_{a}|a\rangle \otimes\left|\phi\left(x-\frac{g T a}{\hbar}\right)\right\rangle .
$$

It is evident from Eq. (1.2.7) that the position of the pointer has been (coherently) shifted by a value that is proportional to the value of observable $\hat{A}$. We assume a Gaussian pointer state at $t=0$

$$
\langle x \mid \phi\rangle=\phi(x)=\left(\frac{1}{2 \pi \sigma^{2}}\right)^{1 / 4} \exp \left(-\frac{x^{2}}{4 \sigma^{2}}\right) .
$$

For such a pointer state, the initial position of the pointer is confined to within a range of positions $\Delta x \propto \sigma$, as set by the uncertainty principle. After the interaction time $T$, all values of $a$ can be resolved provided that the strength of 
the measurement is sufficiently large, $g T a>>\hbar \sigma$. If this condition is satisfied, the initial state of the quantum system $|I\rangle$ has been projected to $|a\rangle$ with a probability of $|\langle a \mid I\rangle|^{2}=\left|\alpha_{a}\right|^{2}$.

A well known example of a projective measurement is the Stern-Gerlach device. Such a device measures the $z$ component of the spin of a spin-1/2 particle via interacting it with a magnetic field directed in the $z$ direction. In this case the measurement operator $\hat{A}=\sigma_{z}$ and he interaction hamiltonian can be written as

$$
\hat{\mathcal{H}}=-g \mu z \sigma_{z} .
$$

Here, $\mu \vec{\sigma}$ is the magnetic moment of the particle, and $g$ is the coupling constant which is set by the strength of the magnetic field in the apparatus. Note that in this case the observable $\hat{A}=\sigma_{z}$ is coupled to the position of the pointer rather to its momentum. Since $z$ is the generator of translations for $P_{z}$, the pointer

will receive a "kick" in a direction set by its spin. The resulting change of the momentum of the operator can be measured by allowing the beam of particle(s) to freely propagate to the far field, where the centroid of the beam correlates with the position of the particle(s) inside the Stern-Gerlach apparatus.

\subsubsection{Weak measurements}

We now review the case of weak measurement, closely following the original treatment by AAV [22]. In a weak measurement, the strength of coupling is not sufficient for resolving the different eigenvalues of $\hat{A}$ due to the uncertainty in the position of the pointer. In this situation, the joint system-pointer state after the interaction can be approximated by using a Taylor series

$$
\begin{aligned}
|\psi(T)\rangle & =\exp \left(\frac{-i g T \hat{A} \otimes \hat{P}}{\hbar}\right)|I\rangle|\phi\rangle \\
& =|I\rangle|\phi\rangle-\frac{i g T}{\hbar} \hat{A}|I\rangle \hat{P}|\phi\rangle+\ldots .
\end{aligned}
$$

We now consider a post-selection on a final state of the quantum system, $|F\rangle$, in order to generalize our measurement scheme further. Conceptually, this 
is equivalent to limiting our analysis to a sub-ensemble of particles that start with an initial state $|I\rangle$, go through the interaction with the pointer for a period of time $T$, and then end up in the final state $|F\rangle$. After projecting the evolved state we find

$$
\begin{aligned}
\langle F| & \exp \left(\frac{-i g T \hat{A} \otimes \hat{P}}{\hbar}\right)|I\rangle|\phi\rangle \\
& =\langle F \mid I\rangle|\phi\rangle-\frac{i g T}{\hbar}\langle F|\hat{A}| I\rangle \hat{P}|\phi\rangle+\ldots .
\end{aligned}
$$

Note that at this stage we have post-selected on the state of the quantum system and we are left with an expression for the state of the pointer. This expression can be simplified by dividing it to $\langle F \mid I\rangle$, to find

$$
\left|\phi_{f i}\right\rangle=|\phi\rangle-\frac{i g T}{\hbar} \frac{\langle F|\hat{A}| I\rangle}{\langle F \mid I\rangle} \hat{P}|\phi\rangle+\ldots .
$$

Recall that in the absence of interaction the post-selection procedure succeeds with a probability of Prob $_{\text {success }}=|\langle F \mid I\rangle|^{2}$. This statement remains approximately correct for weak measurements. It is evident from $\mathrm{Eq}(1.2 .12)$ that the state of the pointer is modified via the quantity $A_{w}=\frac{\langle F|\hat{\mid}| I\rangle}{\langle F \mid I\rangle}$. This quantity is known as the weak value of the observable $\hat{A}$. Below, we show that the weak value is observable through the characterization of the position and the momentum of the pointer. Our analysis closely follows that of Lundeen and Resch [23].

To proceed, we assume the pointer is initially prepared in a Gaussian state described above in $\mathrm{Eq}(1.2 .8)$. We can now calculate the expectation value of 
the position of the pointer to the first order in $g T$ as

$$
\begin{aligned}
\langle\hat{X}\rangle_{f i} & =\left\langle\phi_{f i}|\hat{X}| \phi_{f i}\right\rangle \\
= & -\frac{i g T}{\hbar} \operatorname{Re}\left(\frac{\langle F|\hat{A}| I\rangle}{\langle F \mid I\rangle}\right)\left\langle\phi_{f i}|(\hat{X} \hat{P}-\hat{P} \hat{X})| \phi_{f i}\right\rangle \\
& +\frac{g T}{\hbar} \operatorname{Im}\left(\frac{\langle F|\hat{A}| I\rangle}{\langle F \mid I\rangle}\right)\left\langle\phi_{f i}|(\hat{X} \hat{P}+\hat{P} \hat{X})| \phi_{f i}\right\rangle \\
= & g T \operatorname{Re}\left(\frac{\langle F|\hat{A}| I\rangle}{\langle F \mid I\rangle}\right) .
\end{aligned}
$$

Recall that the symbol \langle\rangle$_{f i}$ signifies an expectation value that is calculated for a state initially prepared as $|I\rangle$ and post-selected on $|F\rangle$. Similarly, we find the expectation value of the pointers' momentum as

$$
\begin{aligned}
\langle\hat{P}\rangle_{f i} & =\left\langle\phi_{f i}|\hat{X}| \phi_{f i}\right\rangle \\
= & -\frac{i g T}{\hbar} \operatorname{Re}\left(\frac{\langle F|\hat{A}| I\rangle}{\langle F \mid I\rangle}\right)\left\langle\phi_{f i}\left|\left(\hat{P}^{2}-\hat{P}^{2}\right)\right| \phi_{f i}\right\rangle \\
& +\frac{g T}{\hbar} \operatorname{Im}\left(\frac{\langle F|\hat{A}| I\rangle}{\langle F \mid I\rangle}\right)\left\langle\phi_{f i}\left|\left(\hat{P}^{2}+\hat{P}^{2}\right)\right| \phi_{f i}\right\rangle \\
= & \frac{\hbar g T}{2 \sigma^{2}} \operatorname{Im}\left(\frac{\langle F|\hat{A}| I\rangle}{\langle F \mid I\rangle}\right) .
\end{aligned}
$$

Thus, we have shown that the shift in the expectation values of $\hat{X}$ and $\hat{P}$ of the pointer are proportional to the real and imaginary parts of the weak value, which is defined as

$$
A_{w}=\frac{\langle F|\hat{A}| I\rangle}{\langle F \mid I\rangle} .
$$

Unlike the result of a projective measurement, a weak value can be beyond the range of eigenvalues of the measured operator [24]. This property, known as weak-value amplification (WVA), has been used before to sensitively measure a variety of effects, such as transverse beam deflections [25, 26, 27, 28], phase differences [29], time delays [30], and nonlinear optical effects [31]. In addition 
to weak-value amplification, the weak values are recently used for directly measuring the quantum wave function $[10,9,8]$. We will further discuss the role of complex weak values in measuring the quantum wave function in a later chapter in this thesis. 


\section{Chapter 2}

\section{Measurement of orbital angular momentum}

\section{$2.1 \quad$ Introduction}

In the previous chapter, we introduced the orbital angular momentum modes of a paraxial light beams. Recently, the OAM modes have been identified as a useful tool in a variety of applications in classical and quantum optics [32, 33, 34, 35]. OAM modes with different $\ell$ values form a large Hilbert space that can be used for encoding information[36]. OAM encoding has been used to increase the channel capacity of a free-space optical link [37]. In addition, the use of a multi-level encoding basis such as the OAM basis increases the tolerance of quantum key distribution (QKD) protocols to eavesdropping [11,38]. In the next chapter, we will outline our implementation of a multilevel QKD system, which is based on OAM encoding. Clearly, applications of this sort require a method of measuring OAM of single photons [5].

Although the two eigenstates of polarization (i.e. spin angular momentum eigenstates) can be separated easily by using a polarizing beam splitter, separation of OAM eigenstates has proven to be much more challenging. The OAM content of an unknown photonic state can be found by performing a series of projective measurements. A variety of experimental methods can achieve this 
goal by using phase plates [32], spatial light modulators (SLM) [39], and q-plates [20]. In a projective measurement, the experimenter passes an unknown photon through a spatial filter designed for detection of a specific value of OAM. This method is analogous to using a polarizer for detecting a certain state of polarization.

Although projective measurements can be used to distinguish between different OAM modes with high accuracy, such measurements are always limited by a success rate of $1 / \mathrm{N}$, where $\mathrm{N}$ is the total number of the OAM modes involved in the experiment. In the previous chapter we established that the information stored in an $N$-dimensional state scales as $\log N$. Considering this, the use of projective measurements results a channel capacity that scale as $\log (N) / N$, a quantity that drops for large values of $N$. To avoid the low success rate, it is desirable to perform the projective measurements on all the $N$ OAM modes simultaneously. This method of performing projections is known as mode sorting. Taking the analogy to measurement of polarization, an OAM mode sorter is analogous to a polarizing beam splitter.

Leach et al. have presented a technique for separating OAM modes at the single photon levels based on using a Mach-Zehdner interferometer with a Dove prism in each arm [40]. This setup allows separation of single photons based on their parity. Although this method work in principle with a $100 \%$ efficiency, separating $N$ modes needs $N-1$ cascaded interferometers. The challenge in designing and aligning such a complicated system considerably limits its applications. In this section we discuss the optical transformation method for sorting OAM modes, and outline its potential for application in a QKD system. 


\section{$2.2 \quad$ Log-polar coordinate transformation}

Berkhout et al. have recently developed a method for sorting OAM modes that employs a Cartesian to log-polar transformation [3]. Under this transformation, the azimuthal phase profile of an OAM mode is mapped to a planar wavefront. As a result, the OAM modes with different quantum numbers $\ell$ are converted to a set of truncated plane waves with wavefront tilts which are proportional to $\ell$. These plane waves are then separated by a single lens.

To realize such a transformation, the input beam's amplitude, $a(x, y)$, is multiplied by a phase-only transmittance function $\phi_{1}(x, y)$. The amplitude of the field in the conjugate Fourier plane can be written in the following form [41]

$$
\begin{aligned}
\tilde{a}(u, v) & =\iint a(x, y) \exp \left[i \phi_{1}(x, y)\right] \exp [-i k(x u+y v) / f] d x d y \\
& =\iint a(x, y) \exp [i k h(x, y)] d x d y .
\end{aligned}
$$

Here, $f$ is the focal length of the lens which performs Fourier transformation, and the quantity $h(x, y)$ is defined via the above relation. For large $k$ values, this integral can be evaluated by using the stationary phase approximation. The results are the same as the solutions of the familiar eikonal equation used in geometrical optics [42]. Namely,

$$
\tilde{a}(u, v)=2 \pi \frac{a(x, y)}{k \sqrt{h_{x x} h_{y y}-h_{x y}^{2}}} \exp i[k h(x, y)+\pi / 2]
$$

for

$$
u=\frac{f}{k} \frac{\partial \phi_{1}(x, y)}{\partial x}, \quad v=\frac{f}{k} \frac{\partial \phi_{1}(x, y)}{\partial y} .
$$

Here $h_{x x}=\partial^{2} h / \partial x^{2}$, etc. For a slowly varying phase-profile, we can ignore the variations of the denominator in Eq. (2.2.2). In this case the input filed distribution will map to the output through the geometrical transformation described in Eq. (2.2.3). If the geometrical map is conformal, the Cauchy-Riemann conditions can be used along with Eq. (2.2.3) to calculate the phase transmitted function $\phi_{1}(x, y)$. As it can be seen in Eq. (2.2.2), the mapping is accompanied 
by an undesired varying phase term. This phase distortion arises from variations in the optical path length and can be removed by placing a second phase plate $\phi_{2}(x, y)$ at the Fourier plane $(u, v)$.

In the Ref. [3] the authors have demonstrated a coordinate mappings of $v=$ $a \arctan (y / x)$ and $u=-a \ln \left(\sqrt{x^{2}+y^{2}} / b\right)$. It is easy to check that this map is conformal. The phase elements are calculated to be $[43,3]$

$$
\begin{aligned}
& \phi_{1}(x, y)=\frac{2 \pi a}{\lambda f}\left[y \arctan \left(\frac{y}{x}\right)-x \ln \left(\frac{\sqrt{x^{2}+y^{2}}}{b}\right)+x\right], \\
& \phi_{2}(u, v)=-\frac{2 \pi a}{\lambda f} \exp \left(-\frac{u}{a}\right) \cos \left(\frac{v}{a}\right) .
\end{aligned}
$$

In the calculations above we have assumed the $(x, y)$ and $(u, v)$ planes are related via a Fourier transformation. Experimentally, a Fourier transformation can be achieved by placing a a lens in between the two planes [44]. Alternatively, two quadratic terms can be added to the phase elements above to eliminate the need for a lens [4]. The resulting phase transmission functions can be realized using SLMs or by creating custom refractive elements [3, 4]. As mentioned earlier, an additional lens is needed in the $(u, v)$ plane to separate the transformed modes. An image of the phase elements placed in a cage system is shown in Fig. (2.1).

The log-polar mapping is based on unitary transformations and hence it can achieve a theoretical power efficiency of unity. In Ref. ([4]) the authors have reported an experimental the power efficiency of $80 \%$. However, the accuracy of measurement is limited by the diffraction-limited size of the transformed modes. In the analysis above we showed that it is possible is to convert OAM modes to plane waves, with the assumption that the plane waves can be perfectly separated using a lens. In reality, however, the OAM modes are converted to a plane wave with finite widths, and thus they are focused onto diffraction-limited, partially overlapping spots. The overlap between such spots leads to error in identifying the neighboring OAM modes. In the next section, we quantify the amount of error and introduce an enhanced mode-sorting technique that can overcome this 
(a)

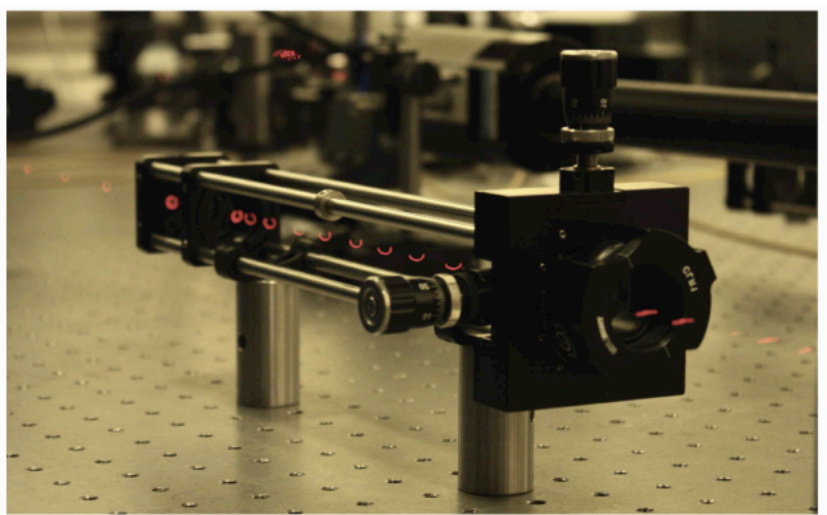

(b)

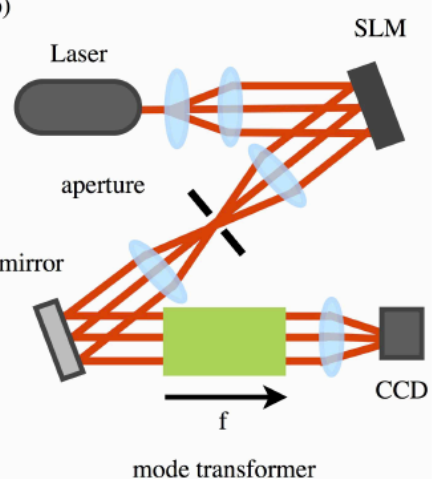

Figure 2.1: The image of the OAM mode sorter device from Ref. [4]. (a) The optical transformation converts the OAM modes to plane waves as they go through the first refractive phase element. The second refractive elements corrects the phase distortion. (b) An SLM is used to realize an $\ell$-forked hologram. A 4 f system is used along with a spatial filter to generate OAM modes. The sorter elements reshape the mode and the lens focuses the separated OAM modes onto a CCD camera.

limitation.

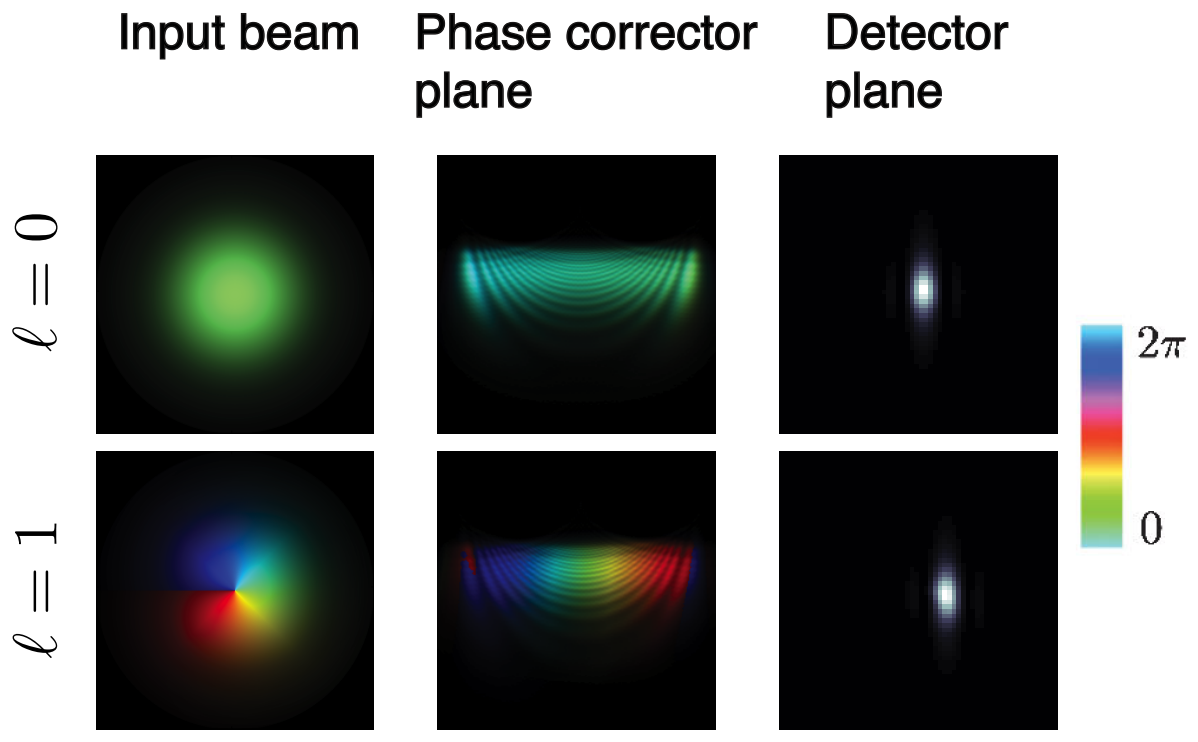

Figure 2.2: Simulated results demonstrating the transformation of the modes undergoing the log-polar coordinate transformation. 


\subsection{Enhanced sorting via refractive beam copy- ing}

In the previous section, we described how the optical transformation method is inherently limited by diffraction. One could guess that, in order to overcome this limitation, it might be possible to use a mapping which converts OAM modes to plane waves with a larger width. It should be emphasized that although the with of the plane waves can be increased by increasing parameter $a$ in Eq. (2.2.4), the tilt angle decreases at the same time proportionally, and as a result the overlap between the neighboring modes remains constant. After going through the sorter elements described by Eq. (2.2.4), the field profile of an unwrapped OAM mode can be written as

$$
U_{\ell}(x)=e^{i \ell x / a} \operatorname{rect}\left(\frac{x}{2 \pi a}\right)
$$

Here, $\operatorname{rect}(x)=1$ for $|x| \leq 1 / 2$ and zero otherwise. Consequently, the field distribution at the focal plane of the lens can expressed as

$$
\tilde{U}_{\ell}\left(x^{\prime}\right)=(2 \pi a) \operatorname{sinc}\left(\frac{x-\Delta \ell}{\Delta}\right)
$$

where $\operatorname{sinc}(x) \equiv \sin (\pi x) /(\pi x)$, and $\Delta=f \lambda /(2 \pi a)$. It can be readily seen from the Eq. (2.3.2) that the overlap between the modes is independent of the parameter $a$ (See Fig. (2.3)). For a single-photon detector, the probability of detection for each mode is proportional to the portion of the intensity that falls into the corresponding spatial bin for an OAM mode. To treat all the OAM modes equally, the spatial bins have to be confined by the borders between the neighboring modes. We define the separation efficiency as the probability of detecting an OAM mode correctly. Consequently, the separation efficiency for the log-polar mapping can be calculated as 


$$
\eta_{S}=\frac{\int_{-1 / 2}^{1 / 2}\left|\frac{\sin (\pi x)}{\pi x}\right|^{2} d x}{\int_{-\infty}^{\infty}\left|\frac{\sin (\pi x)}{\pi x}\right|^{2} d x}=0.774=77.4 \% .
$$

In order to achieve better separation, each OAM beam can be mapped to a repeating structure consisting of multiple periods of the tilted wavefronts. This allows increasing the width of the unwrapped beam while keeping the angle of the tilt constant. As a result of the fixed tilt angle, such modes will be focused to a series of spots that have the same spacing as before but are much narrower $[2,1]$. One way to achieve this is to break the unwrapped beam to $N$ coherent copies. Ideally, such a field distribution can be expressed as $U_{\ell}^{\prime}(x)=e^{i \ell x / a} \operatorname{rect}\left(\frac{x}{2 \pi a N}\right)$, and when focused by the lens yields

$$
\tilde{U}_{\ell}^{\prime}\left(x^{\prime}\right)=\frac{2 \pi a}{\sqrt{N}} \operatorname{sinc}\left(\frac{x-\Delta \ell}{\Delta / N}\right) .
$$

It can be checked from this result that the amount of overlap can be arbitrarily reduced by increasing $N$ (See Fig. (2.3)). We can use an analysis similar to above to calculate the separation efficiency for the case where the log-polar mapping is enhanced with beam copying. For the case of seven copies, the separation efficiency is equal to

$$
\eta_{S}=\frac{\int_{-1 / 2}^{1 / 2}\left|\frac{\sin (7 \pi x)}{7 \pi x}\right|^{2} d x}{\int_{-\infty}^{\infty}\left|\frac{\sin (7 \pi x)}{7 \pi x}\right|^{2} d x}=0.974=97.4 \%
$$

In addition to sorting the OAM modes, our technique can be used to separate a set of modes known as the angular (ANG) modes. These modes are defined as a superposition of OAM modes

$$
\theta_{j}(r, \varphi)=\frac{1}{\sqrt{2 L+1}} \sum_{\ell=-L}^{L} u_{\ell}(r, \varphi) e^{-i 2 \pi j \ell /(2 L+1)}
$$

We will discuss the role of ANG modes in an OAM-based QKD protocol in the next chapter. An efficient method of sorting both OAM and ANG modes is 
required for realizing such a QKD system. We consider the transformation of the ANG modes undergoing a log-polar coordinate mapping. The coordinate transformation acting on mode $\theta_{j}$ yields

$$
\Theta_{j}(x)=\frac{1}{\sqrt{2 L+1}} \operatorname{rect}\left(\frac{x}{2 \pi a}\right) \delta_{L}\left(\frac{x-\Delta_{\theta} j}{a}\right)
$$

where $\Delta_{\theta}=2 \pi a /(2 L+1)$ and $\delta_{L}(u)=\sum_{\ell=-L}^{L} e^{i \ell u}$ is the Dirichlet kernel. The intensity pattern has a peak at $x=\Delta_{\theta} j$ and its first zero occurs at $x=\Delta_{\theta}(j \pm$ 1). Thus, as with the OAM modes, ANG modes can be sorted using position information. However, the spots corresponding to neighboring modes overlap spatially, leading to crosstalk in detection. It is straightforward to show that a detection scheme based on the intensity confinement of ANG mode has the rate of error of $23 \%$.

Analogous to the trick we used with the OAM modes, we can modify the spatial bandwidth of these modes in such a way that the performance of the sorting improves. That is, using a lens of focal length $f$, we focus the transformed angular mode, yielding the field $\tilde{\Theta}_{j}^{\prime}\left(2 \pi x^{\prime} / \lambda f\right)$. If we now coherently split this field into an array of $N$ orders spaced by $(2 L+1) \lambda f /(2 \pi a)$ and refocus this field using another lens of focal length $f$, the resulting field will be identical to the field in Eq. (2.3.7) but instead the Dirichlet function will contain $N(2 L+1)$ terms. When $N=2 n+1$ is odd, the field is written as

$$
\Theta_{j}^{\prime}(x)=\frac{1}{\sqrt{N(2 L+1)}} \operatorname{rect}\left(\frac{x}{2 \pi a}\right) \delta_{n L}\left(\frac{x-\Delta_{\theta} j}{a}\right)
$$

which has a narrower width than the field in Eq. (2.3.7) by a factor of $N$. Consequently, low crosstalk can be achieved in the sorting of ANG modes by choosing a sufficiently large number of copies, $N$.

We have used a periodic phase-only hologram known as a fan-out element to make multiple coherent copies of the unwrapped OAM modes. The phase 
structure of such a device can be described as [45]

$$
\Psi_{2 N+1}(x)=\tan ^{-1}\left(\frac{\sum_{m=-N}^{N} \gamma_{m} \sin \left[(2 \pi s / \lambda) m x+\alpha_{m}\right]}{\sum_{m=-N}^{N} \gamma_{m} \cos \left[(2 \pi s / \lambda) m x+\alpha_{m}\right]}\right)
$$

where $2 N+1$ is the number of copies of the beam, $s$ is the angular separation between them, and $x$ is the transverse dimension along which the copies are made (See Fig. (2.4) panels (a) and (b)). Here, $\gamma_{m}$ and $\alpha_{m}$ are relative phase and intensity parameters associated with different diffraction orders (See Table (2.1)). These parameters can be optimized using numerical or analytical methods to uniformly distribute more than 99 percent of the incident light between the copies $[45,46]$.

\begin{tabular}{|c|c|c|c|c|c|c|c|c|c|}
\hline Diffraction order $m$ & -3 & & -2 & $\begin{array}{l}-1 \\
\end{array}$ & ( & \multicolumn{2}{|c|}{1} & 2 & 3 \\
\hline$\gamma_{7}(m)$ & 1.2 & & 1.45 & 1.28 & 1 & \multicolumn{2}{|c|}{1.28} & 1.45 & 1.24 \\
\hline$\alpha_{7}(m)(\mathrm{rad})$ & 7.0 & & 1.89 & -0.99 & ( & \multicolumn{2}{|c|}{-0.99} & 1.89 & 7.03 \\
\hline Diffraction order $m$ & -4 & -3 & -2 & -1 & 0 & 1 & 2 & 3 & 4 \\
\hline$\gamma_{9}(m)$ & 1.03 & 0.943 & 0.963 & 0.971 & 1 & 0.971 & 0.963 & 0.943 & 1.03 \\
\hline$\alpha_{9}(m)(\mathrm{rad})$ & 1.41 & 3.03 & 5.57 & 0.72 & 0 & 0.72 & 5.57 & 3.03 & 1.41 \\
\hline
\end{tabular}

Table 2.1: Parameters for fan-out elements. (top) Grating for creating seven copies. (bottom) Grating for creating nine copies.

Similar to the case of geometrical mapping discussed above, a second phase plate is needed to compensate the introduced phase distortions in the Fourier plane of the fan-out element. The fan-out element can be combined with the first element of the log-polar sorter to directly convert an OAM beam to a plane wave which is $N$ times larger. The combination can be though of as a 1-to- $N$ extension of the log-polar map. Similarly, the secondary phase elements can be combined to form a single phase element at the Fourier plane of the input beam. For practical reasons however, it is easier to cascade the fan-out hologram rather than adding it directly to the geometrical map. This is partially due to the fact 
that the repeating structure of the hologram asks for a set of different techniques to implement as compared to the slowly varying shape of the reformatter.

Figure (2.5) shows the schematic diagram of our experimental realization of the enhanced sorter [1]. In our experiment, we generate OAM modes using a spatial light modulator (SLM) and a 4 f system of lenses [47]. Figure (2.6) demonstrates the intensity profiles of some of the modes along with the hologram used for generating each mode. The OAM beam then propagates through the refractive elements which perform the log-polar coordinate transformation [4]. A fan-out element realized on SLM1 generates multiple copies of this truncated plane wave mode. For clarity Fig. 2.5 shows a fan-out element only creating 3 copies. However, we have used a fan-out design which creates 7 copies of the beam [45]. SLM2 is used to correct the relative phase between these copies at the Fourier plane of the fan-out element. The holograms for the fan-out element and the corresponding phase corrector are shown in Fig. 2.4 (a). Finally, a lens is used to focus the extended plane-wave to a spot, where a charge-coupled-device (CCD) camera measures its intensity profile.

We have measured OAM modes with quantum numbers of $\ell=-12$ to $\ell=12$ using our method. We divide the area on the CCD into 25 non-overlapping adjacent spatial bins. Each of the bins corresponds to the central position of the spot resulting from the transformation of an OAM mode. Figure (2.7) shows the experimental intensity profile for each transformed OAM mode captured by the CCD camera. From the data, we have calculated the average probability for correctly detecting an OAM mode to be $92.1 \pm 0.7$ percent. This is slightly lower than the theoretical maximum of 97 percent which was calculated above. We attribute the difference to the non-ideal behavior of a phase-only fan-out element, undesired effects from pixelation of the SLMs, and small misalignments in the system. However, this value is substantially higher than the maximum separation efficiency of 77 percent achieved by just the log-polar mapping method $[?]$.

A variation of the sorting method described above can be used to sort ANG modes. Since the unwrapped OAM and ANG modes are related via a Fourier transform in the horizontal direction, we can sort ANG modes by removing the 
Fourier-transforming lens L1 after the second refractive element R2. Instead the unwrapped mode is imaged directly into the fan-out element. We use a fanout element creating 9 copies (See Table (2.1)). The holograms for the fan-out element and the corresponding phase corrector are shown in Fig. (2.4), panel (b). The ANG modes are transformed to a series of separated spots at the focal plane of the lens L2. Figure (2.8) shows the experimental intensity profile for each transformed ANG mode captured by the CCD camera. The average probability for correct detection of an ANG mode in this set is measured to be $92.7 \pm 0.6$ percent. 


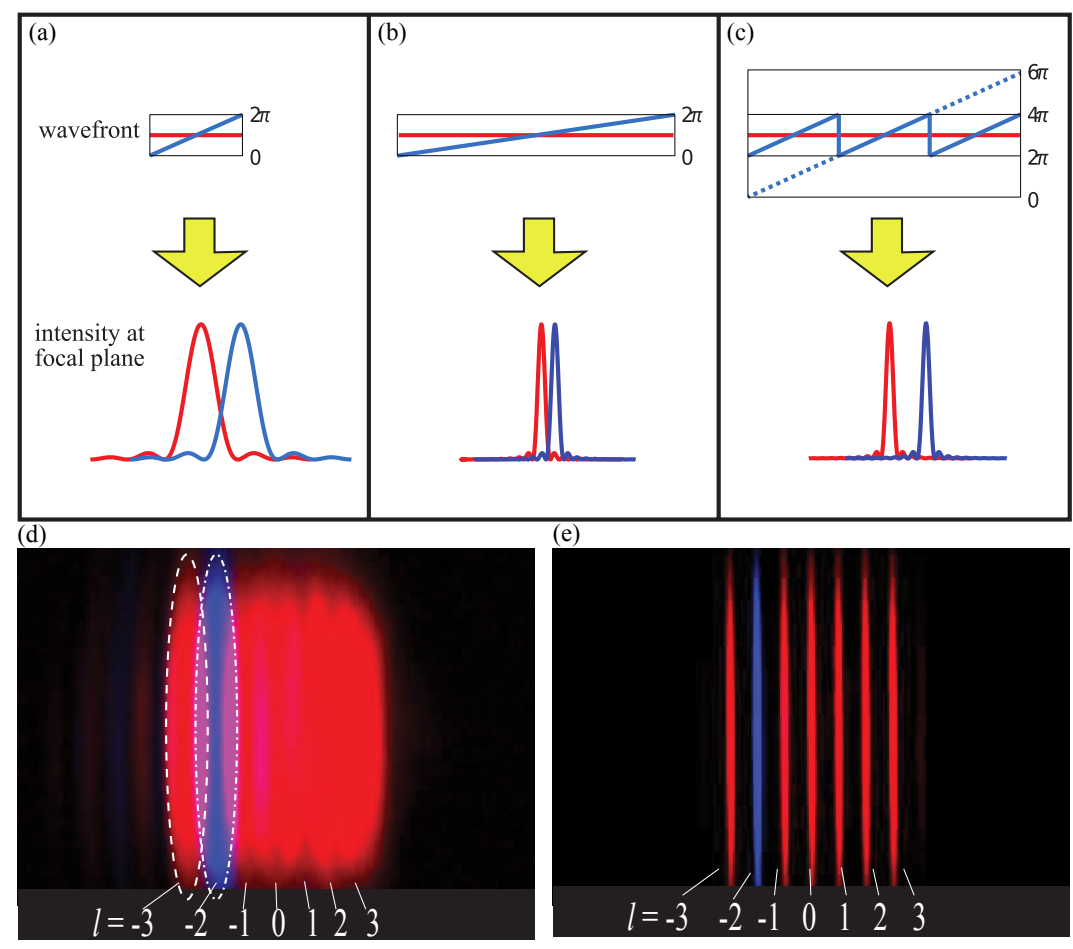

Figure 2.3: Effect of beam copying on mode overlap. (a) Two tilted plane waves resulting from the transformation of two neighboring OAM modes are focused to overlapping spots by a lens. (b) Magnified plane waves can be focused to narrower spots that are spaced closer to each other albeit with no change in overlap. (c) Multiple coherent copies of the transformed plane waves are placed next to each other resulting in focused spots with reduced overlap. (d) Experimental data for OAM modes focused after the log-polar mapping. The data for seven modes $\ell=-3: 3$ are super-imposed on top of each other. The $\ell=-2$ mode is displayed in false color to mark the overlap with the neighboring modes. (e) Experimental data for the modes from part $d$ when enhanced by making seven copies in the Fourier domain. Results are from Ref. [1]. 
(a)
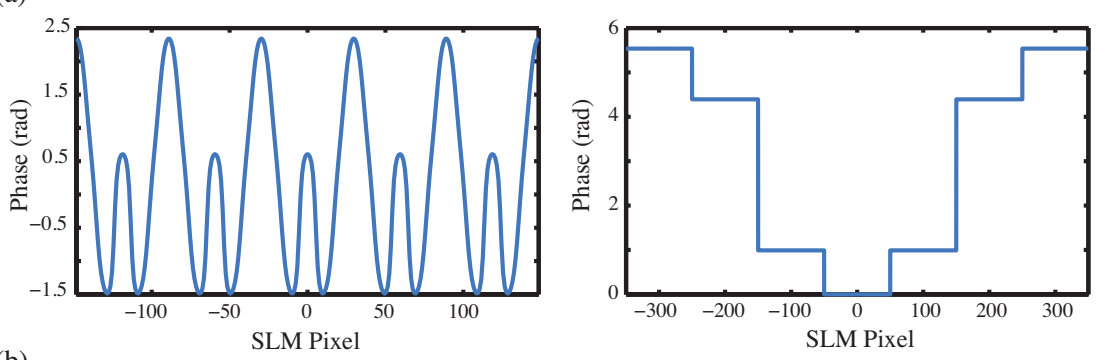

(b)
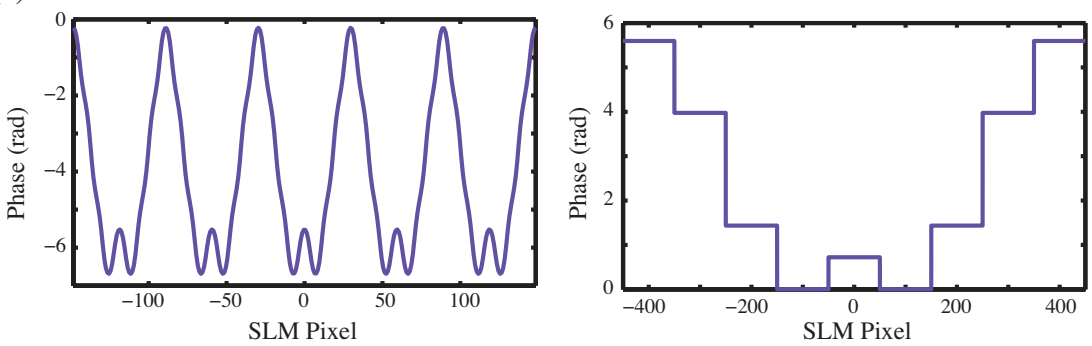

Figure 2.4: Phase profiles for diffractive beam copying. (a) The phase grating for creating seven copies (shown on the left) along with the corresponding phase profile of the phase-correcting element (shown on the right). (b) The fan-out and phase corrector phase profiles for creating nine copies. 


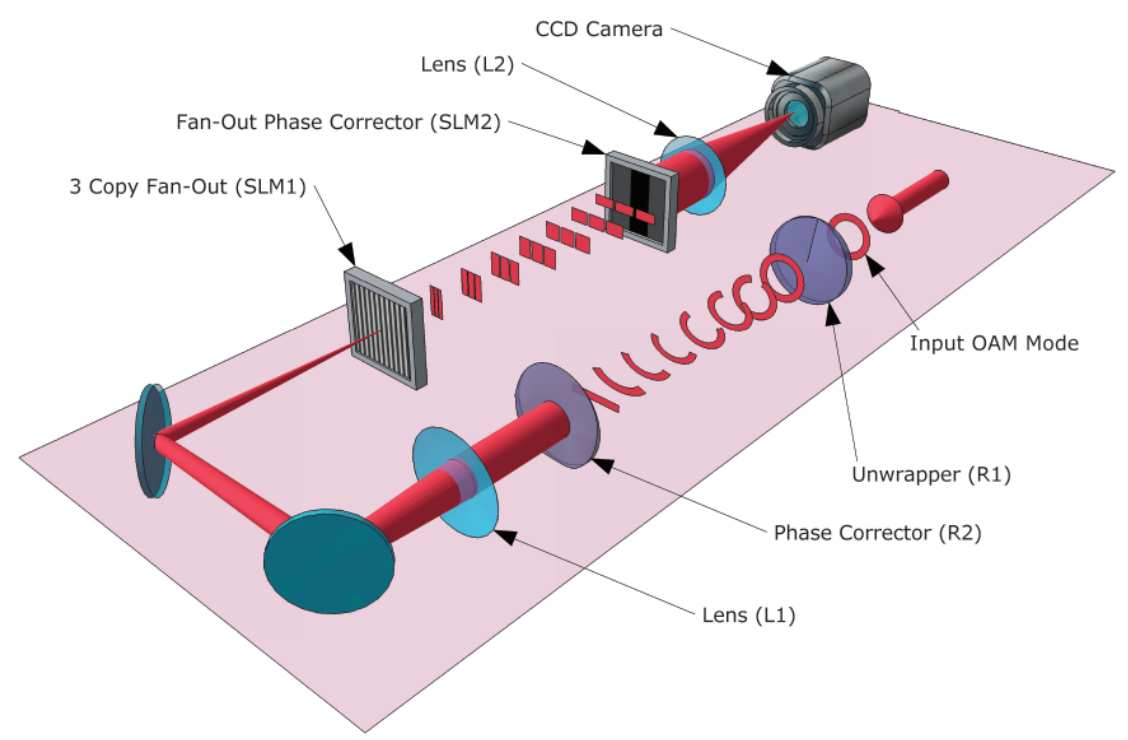

Figure 2.5: The schematic of the mode sorting set-up from Ref. [1]. The optical transformation converts the OAM modes to plane waves as they go through the first and second refractive phase elements. An SLM is used to create multiple copies of the unwrapped beam. A lens focuses the resulting wide beam into a spot, after the phase distortions are removed by the second SLM. (To simplify the demonstration, a fan-out element creating only three copies has been displayed. For further simplification, we have also eliminated the Fourier transform lens between the fan-out and the phase corrector element.)

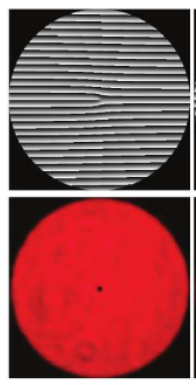

$\ell=-1$

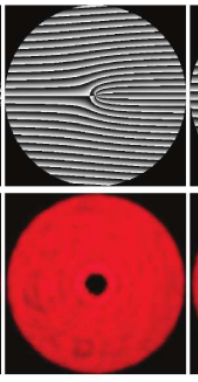

$\ell=5$

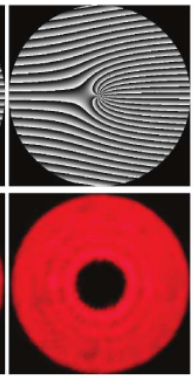

$\ell=12$

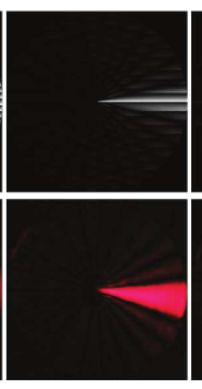

$j=0$

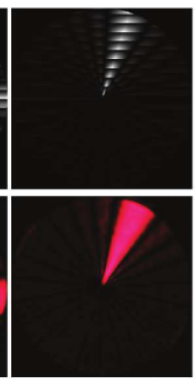

$j=5$

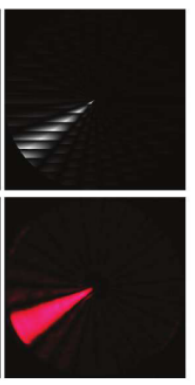

$j=15$

Figure 2.6: Generation of OAM and ANG modes. Examples of holograms (top line) for generating OAM and ANG modes along with the intensity profile (bottom line) of the resulting spatial modes. 


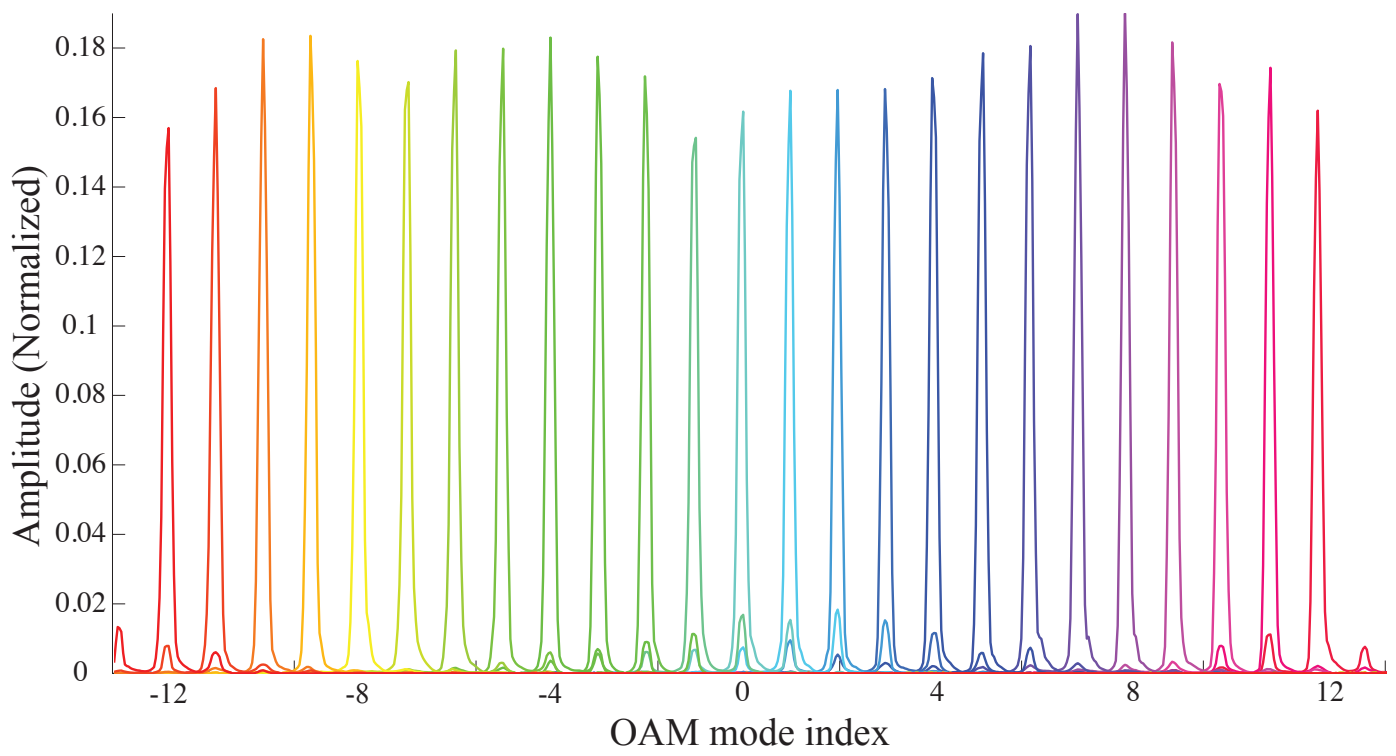

Figure 2.7: Intensity profile of the transformed OAM modes. The incident power in each OAM mode (summed along the vertical axis) as a function of the horizontal coordinate on the CCD camera. The center of each mode is labelled with the corresponding OAM mode index. The sum of the total power in each mode is normalized to unity.

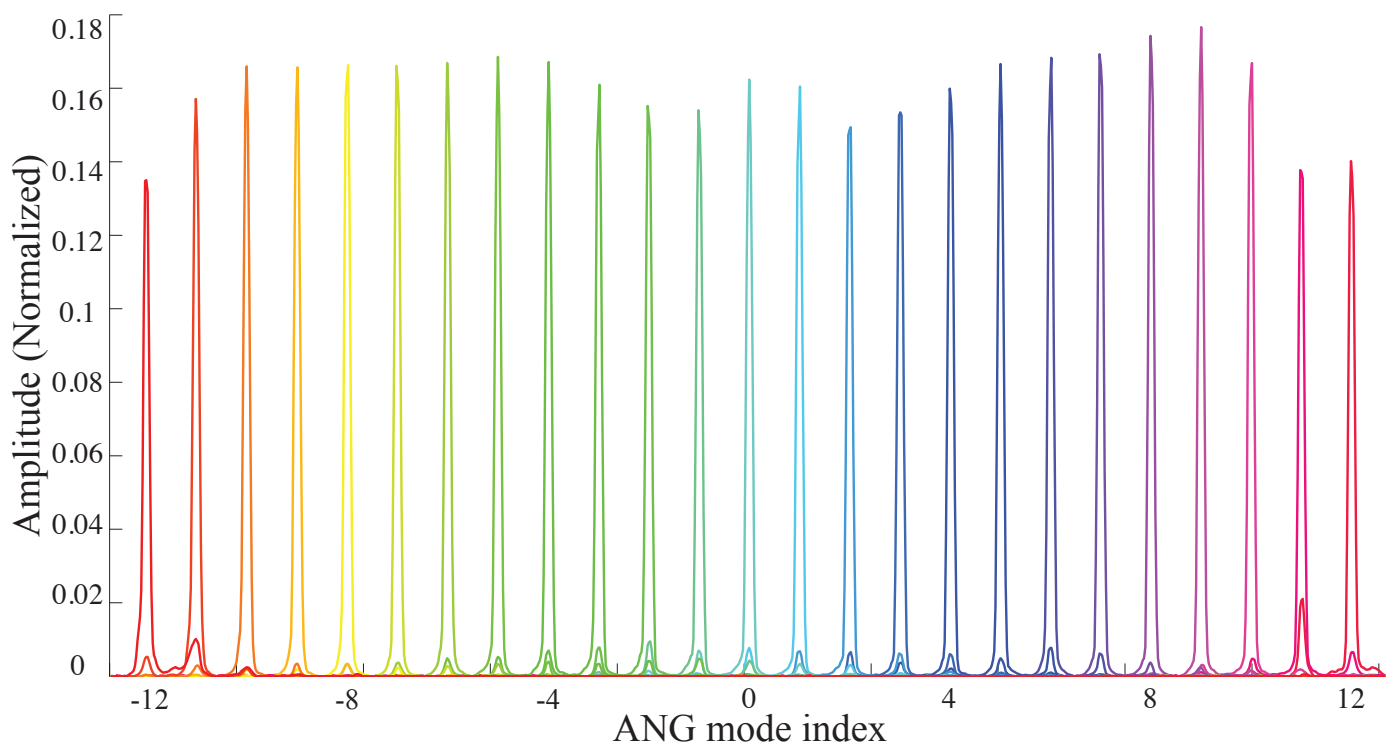

Figure 2.8: Intensity profile of the transformed ANG modes. The total incident power in each ANG mode as a function of the horizontal coordinate on the CCD camera. The center of each mode is labelled with the corresponding ANG mode index. Sum of the total power in each mode is normalized to unity. 


\subsection{Characterization of the mode sorter for ap- plication in QKD}

A common measure to quantify the information capacity of a communication link is the mutual information between the sent and received symbols. This quantity is defined as

$$
I(Y, X)=\sum_{i, j} P\left(y_{j}, x_{i}\right) \log _{2}\left[\frac{P\left(y_{j}, x_{i}\right)}{P\left(x_{i}\right) P\left(y_{j}\right)}\right] .
$$

For the specific case of a QKD system, $x_{i}$ is the event of sending a photon with the OAM value of $\ell_{i}$ and $y_{j}$ is the event of detecting a photon with the OAM value of $\ell_{j}$. We assume a uniform probability for sending $N$ modes, that is, we set $P_{x}$ equals $1 / N$ for all $x$. The joint probability of detection $P\left(y_{j}, x_{i}\right)$ can be calculated from the conditional probability $P\left(y_{j} \mid x_{i}\right)$ by using the Bayes' rule

$$
P\left(y_{j}, x_{i}\right)=P\left(y_{j} \mid x_{i}\right) P\left(x_{i}\right)=\frac{1}{N} P\left(y_{j} \mid x_{i}\right) .
$$

We use mutual information to characterize the performance of the sorter in a QKD protocol. In our detection scheme we spatially bin the detector plane in order to distinguish each mode. In this case, the conditional probability $P\left(y_{j} \mid x_{i}\right)$ is proportional to incident power from the input mode $i$ on the bin dedicated to detection of the mode $j$ (For patterns of the detected modes, see Fig. (2.7) and Fig. (2.8)). The proportionality constant can be simply found by imposing the probability normalization condition $\sum_{j} P\left(y_{j} \mid x_{i}\right)=P\left(x_{i}\right)=\frac{1}{N}$. We have estimated the conditional probability matrix for detection of $25 \mathrm{OAM} / \mathrm{ANG}$ modes by sending a known OAM/ANG mode through the system and measuring the detected power in all 25 bins (See Fig. (2.9)).

We denote the conditional probabilities measured from this method as $P\left(y_{j} \mid x_{i}\right)=$ $S_{j i}$. Using the values of $S_{j i}$ matrix, the mutual information between the trans- 
(a)

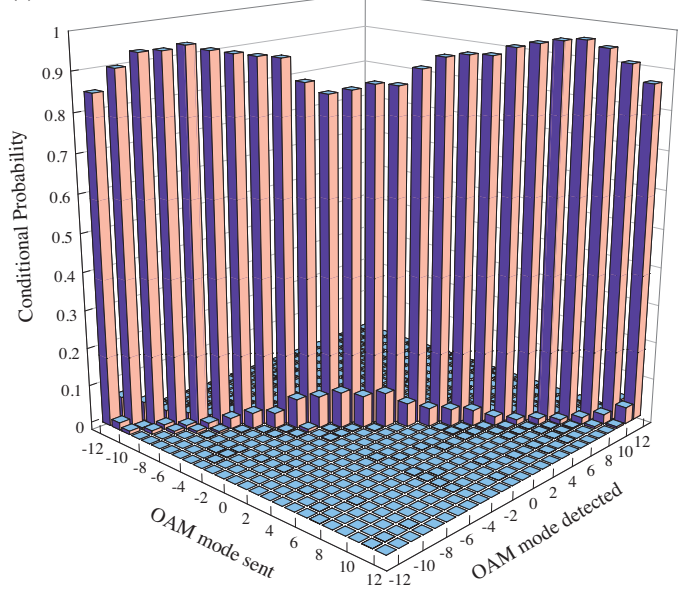

(b)

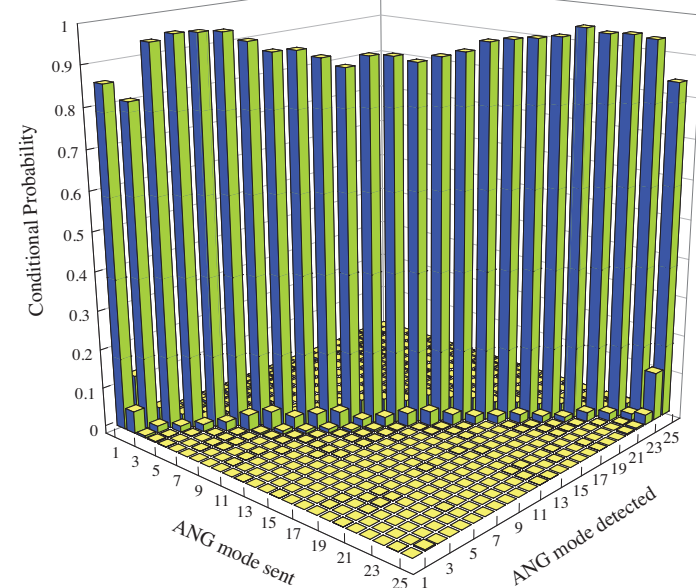

Figure 2.9: The conditional probability of detection. The horizontal axes indicate the indices for the sent and received (a) OAM modes and (b) ANG modes.

mitted and the detected modes can be calculated as

$$
I(\text { bits per detected photon })=\frac{1}{N} \sum_{i, j=1}^{N} S_{j i} \log _{2}\left[\frac{N S_{j i}}{\sum_{i} S_{j i}}\right] .
$$

We have used the conditional probability matrix $S_{j i}$ to calculate the mutual information for OAM and ANG bases. For the OAM modes, the mutual information is calculated to be 4.18 bits per detected photon, as compared with the theoretical upper limit of 4.64 bits per detected photon for a set of 25 modes. Similarly, the mutual information for the set of ANG modes equals 4.16 bits per detected photon, demonstrating the capability of sorting ANG modes with approximately the same separation efficiency as that of the OAM modes.

We have plotted the mutual information for OAM basis sets with with different dimensions on Fig. (2.10). For comparison, we have also plotted the theoretical upper bound for the mutual information of a system employing $N$ OAM modes, which equals $\log _{2}(N)$. We provide the values of mutual information calculated from theory for a perfect log-polar coordinate mapping as well as those from perfect log-polar mapping combined by beam copying on the same plot.

Note that the normalization condition described above excludes the loss in the channel. As a result, the mutual information calculated above only quantifies 


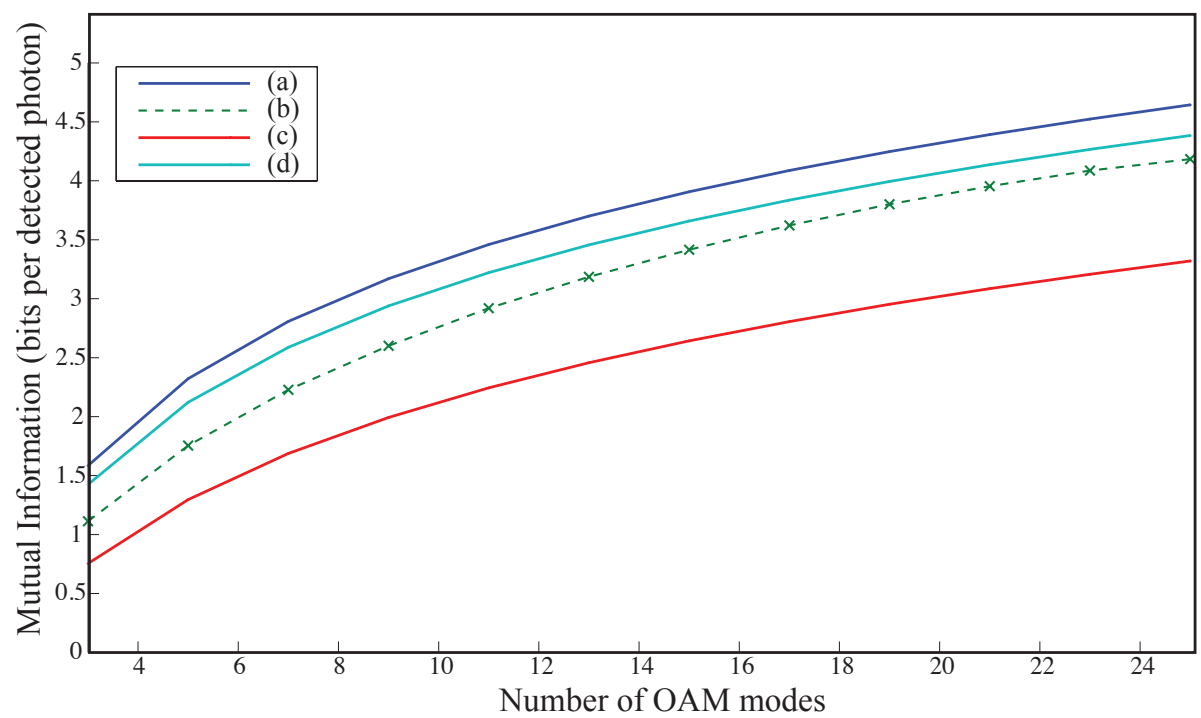

Figure 2.10: Mutual information as a function of the number of the modes. Mutual information as calculated for a) ideally separated modes, b) data from this experiment, c) perfect log-polar mapping, and d) perfect log-polar mapping combined with beam copying. All values are in bits per detected photons

the degree of cross-talk and for this reason we have expressed it in units of bits per detected photon. Since our method relies on phase-only optical elements, in principle it can achieve a power transmission of unity. Thereby, the mutual information expressed in units of bits per detected photon correspond to an ideal realization of the mode sorter device with zero loss.

To achieve a more realistic characterization of the performance of the sorter, we should take into account the loss as well as the cross-talk in the calculation of the mutual information. Due to the presence of loss in a realistic channel, sometimes the transmitted photons lead to no detection event in the receiver's side. In this situation, the failure to detect any mode should be treated as an alternative possible event for the receiver [48]. However, it is easy to check that if the value of the loss is equal for all the modes, the no-detection event contains no information about the transmitted mode and hence it can be discarded from the calculation of the mutual information. In this case, the only effect of loss is in reducing the probability of successfully detecting each mode.

If the transmitted photon is lost in the communication channel by a prob- 
ability of $P_{L}$, a successful transmission will have a probability of $P_{T}$, where $P_{T}=1-P_{L}$. In this case, the joint probability of detection $P\left(y_{j}, x_{i}\right)$ for each mode is calculated as

$$
P\left(y_{j}, x_{i}\right)=P\left(y_{j} \mid x_{i}\right) P\left(x_{i}\right)=\frac{1}{N} P_{T} S_{j i}
$$

where $S_{j i}$ are measured as before. Substituting the joint probability values in Eq. (S8) we have

$$
I(\text { bits per launched photon })=\frac{1}{N} \sum_{i, j=1}^{N} P_{T} S_{j i} \log _{2}\left[\frac{N P_{T} S_{j i}}{\sum_{i} P_{T} S_{j i}}\right]
$$

This can be further simplified as

$$
I(\text { bits per launched photon })=P_{T} \times I(\text { bits per detected photon }) .
$$

This relation has a simple interpretation. It states that the mutual information between the transmitted and the detected modes is scaled down by the the overall power transmission efficiency (i.e., the optical throughput) of the communication link.

We have measured the overall power efficiency of our sorter system to be about 15 percent. Using this number, our system transfers 4.18 bits per detected photon and $4.18 \times 0.15=0.63$ bits per launched photon for OAM modes. Similarly, the ANG modes are transferred and separated with a mutual information of 4.16 bits per detected photon and $4.16 \times 0.15=0.62$ bits per launched photon. We emphasize once more that the loss in our system is a consequence of the specific experimental realization we have utilized, and is not inherent to our sorting method. Straightforward technological improvements could lead to a significant increase in the number of bits per launched photon. Manufacturing of the fan-out and its phase corrector in the form of refractive elements can achieve power transmission efficiencies close to unity. In fact, fan-out elements with efficiencies of more than $95 \%$ are commercially available. Alternatively, 
the required elements can be realized using new generation of SLMs which can achieve diffraction efficiencies of more than $90 \%$. 


\subsection{Summary}

In this chapter, we briefly reviewed different methods of sorting OAM modes and compared their performances. We then provided an in-depth study of the coordinate mapping technique as the primary method for separation of OAM modes at the single-photon level. We quantitatively analyzed the limitations of the coordinate mapping method, and subsequently proposed a modified sorting technique that uses coherent beam copying for reducing the overlap among the transformed modes. We presented experimental data that demonstrates a mode separation efficiency of $92.4 \%$ for our enhanced technique. This value is evidently superior to the $77 \%$ separation efficiency of the coordinate mapping method. Additionally, we reported on a variant of our technique that can separate the angular position (ANG) modes. We quantified the performance of the enhanced mode sorter for a set of 25 OAM and ANG modes. From experimental data, we calculated a channel capacity of 4.16 (bits per photon) for the OAM and 4.18 (bits per photon) for the ANG modes. These results establish the possibility of performing accurate projections on the OAM and ANG modes, which is a crucial stage of an OAM-based QKD protocol. In the next chapter, we outline our experimental implementation of a multilevel QKD system that is based on the sorting method described in this chapter. 


\section{Chapter 3}

\section{Quantum cryptography with twisted photons}

\subsection{Introduction}

First introduced in 1984 by Bennett and Brassard, quantum key distribution (QKD) is a method for distributing a secret key between two parties $[49,50]$. Due to a fundamental property of quantum physics known as the no-cloning theorem, any attempt made by a third party to eavesdrop inevitably leads to errors that can be detected by the sender and receiver $[51,52]$. QKD schemes conventionally use qubits for encoding information, often realized with the polarization of single photons. Such systems are easily implemented because technology for encoding and decoding information in a qubit state-space is readily available today, enabling system clock rates in the $\mathrm{GHz}$ regime $[53,54,55]$. Recently, the spatial degree of freedom of photons has been identified as an extremely useful resource for transferring information $[34,39]$. The information transfer capacity of classical communication links has been increased to more than one terabit per second using spatial-mode multiplexing [37]. In addition, it has been theoretically shown that employing multilevel quantum states (qudits) can increase the robustness of a QKD system against eavesdropping [56, 57, 11, 58]. Although the majority of high-dimensional QKD schemes so far have employed time-bin 
encoding for increasing the alphabet size $[59,60,61,62]$, it is expected that spatial-mode encoding can be alternatively used to enhance the performance of a QKD system considering the recent advances in free-space OAM communication, .

Similarly, a number of studies have investigated the benefits of employing OAM modes in quantum cryptography $[63,64,65]$. Recently, rotation-invariant OAM vector modes have been used for performing QKD in a two-dimensional state space [66]. Although this method offers an advantage in terms of optical alignment, it fails to utilize the large information capacity of the OAM basis. The complete realization of a high-dimensional QKD system with OAM has remained impractical up until now mostly due to the difficulty in efficiently sorting single photons in the OAM basis. Additionally, any realistic application requires a fast key-generation rate that cannot be achieved with most of the common methods for generating OAM modes.

In the previous chapter, we discussed different methods of measuring the OAM of light, and we introduced a method of sorting the OAM of single photons. In this chapter, we report on our implementation of a QKD system that is based on OAM-encoding. We start with a brief study of free-space communication with spatial modes of light. We then review the BB-84 protocol and generalize it for multilevel quantum states. We describe a proof-of-principle experiment that demonstrates the feasibility of performing high-dimensional quantum cryptography with OAM modes. Finally, we discuss the limitations of our approach and explore possibilities for further development of this avenue of research. 


\subsection{Free-space communication with spatial modes}

The feasibility of high-dimensional QKD in the spatial domain has been previously demonstrated by encoding information in the transverse linear momentum and position bases [67, 68]. While such encoding schemes provide a simple solution for increasing the information capacity, they are not suitable for operation in long-haul optical links. Upon propagation over long distances, diffraction creates a varying transmission loss for different spatial frequencies that results in mixing of spatial modes $[69,70]$. Cross-talk increases the quantum symbol error rate (SER) and fundamentally limits the secure key rate of a QKD system. As we show below, the adverse effects of diffraction can be alleviated by employing spatial modes that carry OAM.

Consider a typical free-space communication link as depicted in Fig. (3.6). Due to diffraction, a spatially confined beam generated in the transmitting aperture spreads upon propagation. The area of the beam over which most of its energy is confined can be calculated at any plane using diffraction theory. However, after propagating beyond the near field, the beam has long (transverse) tails that continue toward infinity [44]. Since the receiving aperture has a finite size, a portion of energy of the beam will be lost in the detection process. Additionally, the strength of the induced loss is dependent on the form of the transmitted field. As a result, a set of initially orthogonal fields in the transmitting aperture will no longer form an orthogonal set at the receiving aperture, and hence it is impossible to perfectly discriminate among them.

The input-output characteristics of a free-space optical link can be described using the Rayleigh-Sommerfeld diffraction formula, which describes the freespace propagation of an arbitrary scalar field as

$$
\Psi_{\text {out }}\left(\mathbf{r}_{R}\right)=\int \Psi_{\text {in }}\left(\mathbf{r}_{T}\right) K\left(\mathbf{r}_{T}, \mathbf{r}_{R}\right) d^{2} \mathbf{r}_{T},
$$

in which $\Psi_{\text {in }}\left(\mathbf{r}_{T}\right)$ and $\Psi_{\text {out }}\left(\mathbf{r}_{R}\right)$ are the electric fields in the transmitting and the 


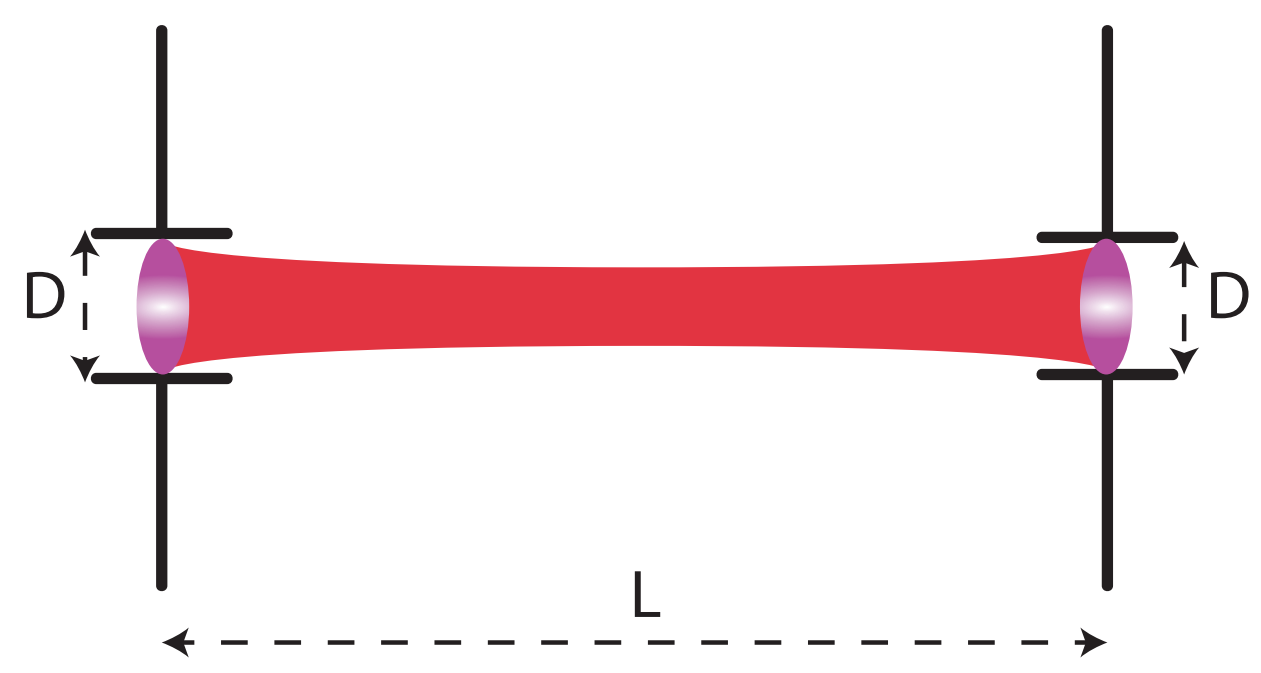

Figure 3.1: Schematic diagram of a free-space communication link.

receiving apertures. The propagation kernel $K\left(\mathbf{r}_{T}, \mathbf{r}_{R}\right)$ can be written as [44]

$$
K\left(\mathbf{r}_{T}, \mathbf{r}_{R}\right)=-\frac{1}{2 \pi} \frac{\partial}{\partial z} \frac{\exp \left(i k\left|\mathbf{r}_{T}-\mathbf{r}_{R}\right|\right)}{\left|\mathbf{r}_{T}-\mathbf{r}_{R}\right|}
$$

Eq. (3.2.1) related the fields in the two apertures via a linear transformation. Using Dirac notation, this relation can be written simply as $\left|\Psi_{\text {out }}\right\rangle=\hat{P}\left|\Psi_{\text {in }}\right\rangle$, where $\hat{P}$ is the propagation operator. Note that the propagation kernel as defined above represents a unitary transformation. In order to calculate the detected field, however, we must take into account the finite size of the apertures. This imposes the condition $K\left(\mathbf{r}_{T}, \mathbf{r}_{R}\right)=0$ for $r_{R}>R_{1}$ and $r_{T}>R_{0}$, where $R_{0}$ and $R_{1}$ are the radii of the transmitter's and the receiver's apertures, respectively. As a result of imposing this constraint, the propagator is no longer a unitary operator. A non-unitary transformation does not preserve inner products. Hence, a set of orthogonal modes generated in the transmitting aperture are not necessarily orthogonal within the receiving aperture, that is,

$$
\begin{array}{r}
\left\langle\Psi_{m-\text { out }} \mid \Psi_{n-\text { out }}\right\rangle=\left\langle\hat{P} \Psi_{m-\text { in }} \mid \hat{P} \Psi_{n-\text { in }}\right\rangle \\
=\left\langle\Psi_{m-\text { in }}\left|\hat{P}^{\dagger} \hat{P}\right| \Psi_{n-\text { in }}\right\rangle \neq 0 .
\end{array}
$$


Note that since $\hat{P}$ is not unitary, $\hat{P}^{\dagger} \hat{P}$ need not constitute the identity matrix.

The problem of finding the optimum set of modes for transmitting energy and information from one finite aperture to another has long been investigated in the context of apodization theory [71] and the theory of communication modes $[72,70]$. The communication modes of an optical link can be found by performing a singular value decomposition (SVD) of the propagation operator. The communication modes, $\left|\Phi_{n}\right\rangle$, can be formally defined using the realtion

$$
\hat{P}^{\dagger} \hat{P}\left|\Phi_{n}\right\rangle=\left|\lambda_{n}\right|^{2}\left|\Phi_{n}\right\rangle
$$

Here, $\left|\lambda_{n}^{2}\right|$ is a coupling coefficient designating the portion of the energy of $\mathrm{n}$-th mode that falls within the receiving aperture [70, 73].

The SVD procedure guarantees that the communication modes form a complete orthonormal set in the receiving aperture. More over, the orthogonality of communication modes are preserved in the receiving aperture in spite of experiencing diffraction loss

$$
\begin{array}{r}
\left\langle\Phi_{m-\text { out }} \mid \Phi_{n-\text { out }}\right\rangle=\left\langle\Phi_{m-\text { in }}\left|\hat{P}^{\dagger} \hat{P}\right| \Phi_{n-\text { in }}\right\rangle \\
=\left|\lambda_{n}\right|^{2}\left\langle\Phi_{m-\text { in }} \mid \Phi_{n-\text { in }}\right\rangle \\
=\left|\lambda_{n}\right|^{2} \delta_{m n} .
\end{array}
$$

The orthogonality in the receiving aperture guarantees that the communication modes can be perfectly discriminated from each other. In terms of designing a QKD system, this property translates to the possibility of building a loss-less mode-sorting device for communication modes. Once the modes are separated, it is straight-forward to channel them to multiple single photon detectors where individual detection events can be registered.

As an example, we review the special case of an optical link with asymptotically large apertures. For such a system, the communication modes in the transmitter can be approximated as spherical waves that are focused onto the receiver's aperture, where they are transformed to diffraction-limited spots [73]. In the receiver, two spots are barely resolvable if they are separated by an amount 


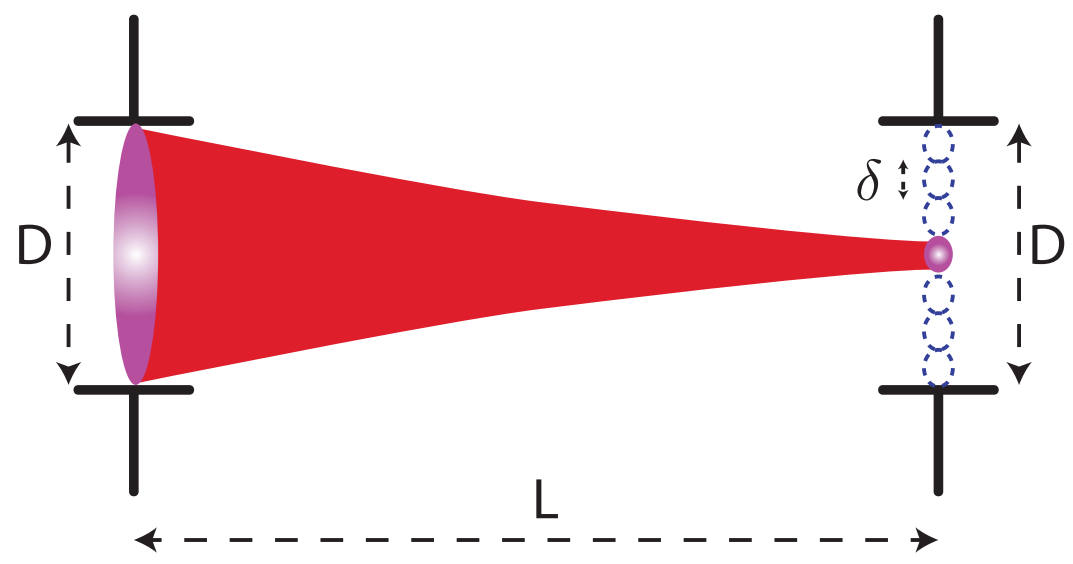

Figure 3.2: Information encoding by the location of distinct spots focused within the receiver's aperture.

$\delta$, which is set by the Rayleigh criterion

$$
\delta \simeq \lambda L / D
$$

Here, $\lambda$ is the wavelength of the laser beam, $D$ is the diameter of the transmitting (and the receiving) aperture, and $\mathrm{L}$ is the propagation distance (See Fig. (3.2)). The area of each spot can be consequently approximated as

$$
\delta^{2} \simeq(\lambda L)^{2} / A
$$

where $\mathrm{A}$ is the area of the transmitting aperture. The total number of spots distinguishable in the receiver aperture is therefore equal to the number of spots that fit within an area of the receiver $A$

$$
N_{F} \simeq A / \delta^{2}=\frac{A^{2}}{(\lambda L)^{2}} .
$$

Here, $N_{F}$, is the product of the Fresnel numbers of the two apertures in the system and for this reason it is often called as the Fresnel number product of the optical link. In the case of a system with arbitrary aperture sizes, calculating the optimum modes for communication and their coupling efficiency has to be done by solving the eigenvalue problem in Eq. (3.2.4). However, the general 
result found above remains valid; that is the number of communication modes that can be efficiently transmitted over a free-space link scales with $N_{F}=\frac{A_{R} A_{T}}{(\lambda L)^{2}}$, $[69,73]$. Therefore, spatial-mode encoding becomes advantageous in an optical link with a large Fresnel number product.

A real-world communication link usually consist of circular components such as lenses, apertures, and mirrors. In this situation, the rotational symmetry of the system can be used to simplify Eq. (3.2.4). Assuming paraxial Fresnel diffraction, it can be shown that the solutions have the form

$$
\Phi_{n, \ell}(\rho, \phi)=\frac{R_{n, \ell}(\rho)}{\sqrt{\rho}} e^{i \ell \phi}
$$

where the radial parts $R_{n, l}(\rho)$ are known as the circular prolate spheroidal wavefunctions (CPSW) [74, 71]. It is evident that the solutions above have a separable dependence on the radial and the azimuthal coordinates. It is straightforward to show that the these modes are eigenstates of the orbital angular momentum operator as suggested by the $e^{i l \phi}$ factor above [14]. In general, any two functions with different OAM values maintain their orthogonality upon propagation, regardless of their radial form [71].

Although the CPSWs are the exact communication modes for optical links with round apertures, their calculation requires a complicated numerical procedure. For this reason, the CPSW modes are often approximated as another set of modes known as the Laguerre-Gaussian (LG) modes. Fortunately, the LG modes can be calculated analytically and they provide a very good approximation for the CPSW modes when Fresnel number of the system is moderately larger than unity [75]. In this case the modes at the transmitter aperture can be written as

$$
\Psi_{l, p}(\rho, \phi)=\frac{4\left(1+4 N_{F}\right)^{1 / 4}}{\sqrt{\pi} D} \mathrm{LG}_{p}^{\ell}\left(\frac{4\left(1+4 N_{F}\right)^{1 / 4}}{D} \rho, \phi\right) e^{-i \frac{k}{2 L} \rho^{2}}
$$




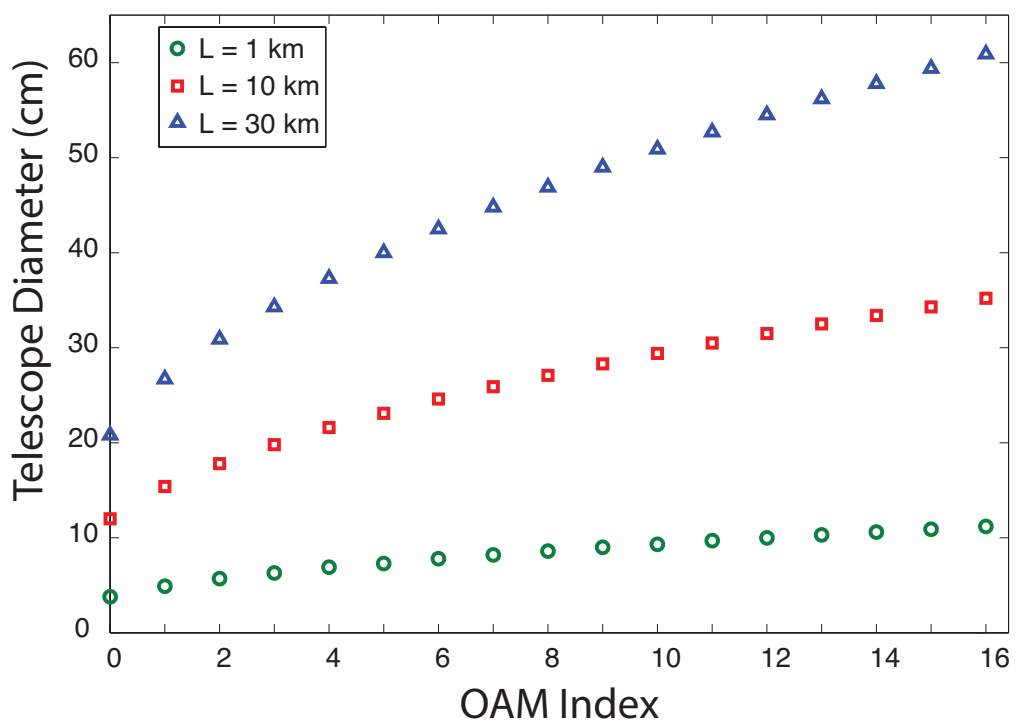

Figure 3.3: Numerical results for the required aperture diameter as a function of the maximum OAM index

where $L G_{p}^{\ell}$ are the Laguerre-Gaussian functions defined as

$$
\mathrm{LG}_{p}^{\ell}(\rho, \phi)=\sqrt{\frac{p !}{(p+|\ell|) !}} e^{-\rho^{2} / 2} \rho^{|\ell|} L_{n}^{|\ell|}\left(\rho^{2}\right) e^{i \ell \phi} .
$$

The $L_{n}^{|\ell|}(z)$ are the generalized Laguerre polynomials, which can be written as

$$
L_{n}^{|\ell|}(z)=\frac{e^{z} z^{-|\ell|}}{n !} \frac{\mathrm{d}^{n}}{\mathrm{~d} z^{n}}\left(e^{-z} z^{n+|\ell|}\right)
$$

We have numerically calculated the transmission efficiency (also known as the coupling efficiency) of the LG modes as a function of the distance between the apertures and the diameter of the transmitting and the reciting apertures. Figure. (3.2) displays the required aperture diameter as a function of the maximum OAM index for different values of propagation distance, $L$. We have found the maximum value of OAM index by requiring the transmission efficiency to be above $95 \%$ for all the transmitted modes. Note that the transmission efficiency is a function of $|\ell|$, and so for a maximum value of $\ell$ one could transmit $2 \ell+1$ 
modes with an efficiency higher than $95 \%$.

It is evident from Fig. (3.2) that a large number of OAM modes (of the order of 20) can be transmitted in an optical link with moderate aperture sizes (of the order of $30 \mathrm{~cm}$ ) over a long distance (of the order $10 \mathrm{~km}$ ). Thus, it is reasonable to investigate the idea of a line-of-sight quantum communication system that is based on encoding information on the OAM modes, given the recent advances in the technology for generation, switching, and the measurement of these modes. In a recent experiment, OAM modes were used for performing classical communication over a 3-km-long free-space optical link [76]. Additionally, the effects of turbulence on propagation of OAM modes, and methods of mitigating it has been a topic of extensive study lately $[77,78,75,7,79,80,81,82]$. In the next part, we outline our implementation of a high-dimensional QKD system based on using OAM modes. 


\subsection{BB-84 QKD with OAM modes}

The goal of encryption is to establish secure communication between two parties, often called as Alice and Bob. The exchange of a secret key is a crucial part of any encryption protocol. In the conventional cryptography schemes, the security of key exchange is based on computational complexity [50]. Quantum key distribution (QKD) provides a radically different means of securely distributing a key whose secrecy is guaranteed by the laws of quantum physics. We explain the underlying principles of QKD by going through different stages of the BB-84 protocol, the first QKD protocol proposed by Bennet and Brassard in 1984.

BB-84 is based on encoding information on two-level quantum systems, also called as qubits. Physically, a qubit is often realized by using the polarization of a single photon. Here, we have the two legitimate parties Alice and Bob, and a third party Eve, who intends to eavesdrop on Alice and Bob's communication channel. To convey a bit of information, Alice prepares the state of a single photon and sends it to Bob. For each bit value of 1 or 0 , Alice can send a photon whose polarization is randomly selected from two bases and each basis is consisted of two quantum states (See Fig. (3.4)). We use the notation $|H\rangle$ and $|V\rangle$ to refer to the horizontal and vertical polarization states of a photon, respectively. The $\mathrm{H} / \mathrm{V}$ basis is made from the quantum states $|H\rangle$ and $|V\rangle$. Similarly, the D/A basis is constructed from the states $|D\rangle$ and $|A\rangle$, which are defined as

$$
\begin{aligned}
& |D\rangle=\frac{1}{\sqrt{2}}[|H\rangle+|V\rangle], \\
& |A\rangle=\frac{1}{\sqrt{2}}[|H\rangle-|V\rangle] .
\end{aligned}
$$

Upon receiving each photon, Bob randomly chooses the orientation of a polarization filter, such as a polarizing beam splitter, to make a projective measurement of polarization in a basis of his choice $(\mathrm{H} / \mathrm{V}$ or $\mathrm{D} / \mathrm{A})$. While registering the result of his projective measurements, Bob also records his choice of basis (set by the orientation of the polarization filter). Figure. (3.4) illustrate an example 


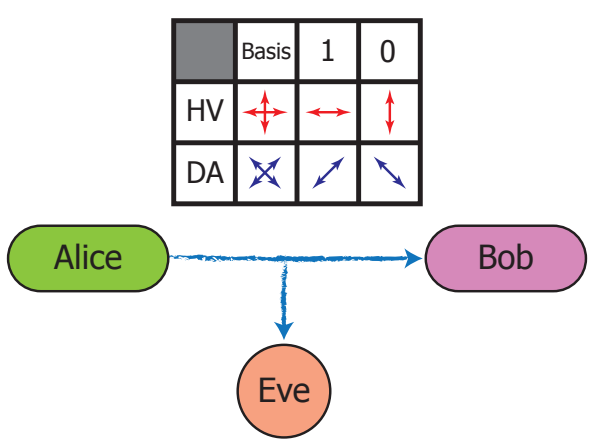

\begin{tabular}{|c|c|c|c|c|c|c|c|c|}
\hline Alice's Random Bit & 0 & 1 & 1 & 0 & 1 & 0 & 0 & 1 \\
\hline Alice's Random Basis & $\uparrow$ & $\uparrow$ & $\searrow$ & $\uparrow$ & $\searrow$ & $\searrow$ & $\searrow$ & $\uparrow$ \\
\hline Alice's Sent Symbol & $\uparrow$ & $\leftrightarrow$ & $\nearrow$ & $\uparrow$ & $\nearrow$ & $\searrow$ & $\searrow$ & $\leftrightarrow$ \\
\hline Bob's Random Basis & $\uparrow$ & $\searrow$ & $\searrow$ & $\searrow$ & $\uparrow$ & $\searrow$ & $\uparrow$ & $\uparrow$ \\
\hline Bob's Measured Symbol & $\uparrow$ & $\searrow$ & $\nearrow$ & $\searrow$ & $\leftrightarrow$ & $\searrow$ & $\leftrightarrow$ & $\leftrightarrow$ \\
\hline Bob's Measured Bit & 0 & 0 & 1 & 0 & 1 & 0 & 1 & 1 \\
\hline Secret shared Key & 0 & & 1 & & & 0 & & 1 \\
\hline
\end{tabular}

Figure 3.4: Left panel: Table shows the MUBs used by Alice for encoding information. Each basis is made from two orthogonal quantum states. Right panel: Table demonstrates a sequence of random numbers sent by Alice and measured by Bob. Notice the perfect correlation of Alice and Bob's sequences when they have used the identical bases. Bob's sequence is statistically independent of that of Alice on the instances when the bases are different.

of a sequence of qubits exchanged between Alice ad Bob. It is evident from the table that Alice and Bob's results are perfectly correlated whenever they choose the same basis. On the contrary, their measurement results are statistically independent from the whenever Alice and Bob choose different bases. Therefore, all Alice and Bob need to do is to publicly announce their choice of basis for each bit in the sequence in order to create a key. Once they have done this, they can only keep the results wherever they have used the same bases and discard the rest. This procedure is called sifting and ideally at this stage Alice and Bob will have two identical copies of a sequence that the can use as a key.

We now consider eavesdropping by Eve. A common eavesdropping strategy is the intercept-resend attack, where Eve initially measures the state of a photon in the channel and then sends a photon prepared in the state set by her measurement's result to Bob. For performing projective measurements, Eve has to randomly choose a basis just like Bob. Since Eve guesses the wrong basis half of the time, she modifies the state of the photons in the channel with a chance of $25 \%$ and will thus introduce errors in the key shared by Alice and Bob. It is thus sufficient for Alice and Bob to monitor the rate of errors in their shared key sequence, by sacrificing a portion of their bit sequences and comparing them together. If the estimated error rate is below 25\%, Alice and Bob can safely assume their protocol is secure. There exists more sophisticated eavesdropping 
strategies, but the quantum no-cloning theorem guarantees that it is impossible for Eve to gain information without disturbing the state of photons in the channel [51]. A more complete analysis provides an upper bound of $14.6 \%$ for the maximum tolerable error rate of the BB-84 protocol [11].

The BB-84 protocol uses a two-level system such as polarization of a photon for encoding information. This limits the information capacity of the quantum channel to a one bit photon and also imposes a tight (14.6\%) bound on the maximum rate of error that can be tolerated without compromising the security of the transmission. Recently, multi-level QKD has been proposed as a generalization of the BB-84 that allows for achieving higher key rates and enhanced security. In this approach, Alice send a number different symbols as opposed to just sending zero and one. This is achieved by encoding the information on a multi-level quantum state, also known as a qudit. This strategy has the advantage that she can encode more information on a single photon. For example an 8 -level system can be used to encode 8 symbols on which is equivalent to 3 bits of information. More generally, the maximum amount of information that such a channel can carry (per photon) can be calculated by using the Shannon entropy formula

$$
I(X)=-\sum_{n} p_{n} \log _{2}\left(p_{n}\right)=\log _{2}(d)
$$

Here, $d$ is the dimensionality of the Hilbert space used for encoding information. The variable $p_{n}$ is the probability of $n$-th outcome of the variable $X$, which is choosing any of the $d$ possible outcomes. Just like the BB-84 protocol, we have assumed all the symbols are used equally frequently. The table if Fig (3.5) shows the information capacity calculated for multiple values of $d$.

In addition to the increase in channel capacity, a multilevel encoding can increase the tolerance of QKD to eavesdropping. Heuristically, this happens since Eve has a higher probability of disturbing a photon while probing a largedimensional quantum state. It is straight-forward to derive the error bound for 
an intercept-resend attack in a $d$-dimensional protocol with $M$ bases as [57]

$$
e_{B}(d, M)=\left(1-\frac{1}{M}\right)\left(1-\frac{1}{d}\right)
$$

Note that Alice can use a larger number of bases $(M)$ in a multilevel protocol. The crucial property in the BB-84 was that choosing the wrong basis would provide a completely random outcome. This idea can be formulated more precisely in terms of mutually unbiased bases (MUBs). Two bases are mutually unbiased if each state in one basis can be written as a superposition of all the states in the other basis with equal weights. In general, the maximum number of MUBs in a $d$-dimensional Hilbert space is equal to $d+1$, where $d$ is a prime number [83]. Equation (3.3.3) shows that increasing the number of symbols $d$, and the number of bases $M$ results in increasing the error bound. Note that although increasing the number of MUBs, $M$, is beneficial in terms of the error bounds, it results in a linear reduction in the efficiency of the sifting procedure. Moreover, increasing the number of MUBs requires the ability for generation and measurement of photons in multiple bases. Considering these factors, we limit our analysis to the simplest case of $M=2$ for the remainder of this chapter. The security of multilevel QKD for the general case of optimal individual attacks (for $M=2$ MUBs) is treated in [11]. We have listed the error bounds for different values of $d$ in the bottom row of the table in Fig (3.5). It is evident that the maximum tolerable error rates increases with $d$, even for the case where only $M=2$ bases are used.

\begin{tabular}{|c|c|c|c|c|c|}
\hline $\mathrm{d}$ & 2 & 3 & 4 & 5 & 8 \\
\hline $\begin{array}{c}\text { Error } \\
(\%)\end{array}$ & 14.64 & 21.13 & 25 & 27.64 & 32.31 \\
\hline $\begin{array}{c}\text { I } \\
\text { (bits) }\end{array}$ & 1 & 1.58 & 2 & 2.32 & 3 \\
\hline
\end{tabular}

Figure 3.5: Channel capacity and error bound calculated for multiple values of $d$, for the case of $M=2$. The error bound values are quoted from [11].

The increased tolerance to eavesdropping, along with the enhanced channel 
capacity motivates the implementation of a multilevel QKD system. We now consider the specific case of QKD with OAM modes. The infinite, multidimensional Hilbert space of OAM modes provides a large basis for encoding information. We choose our primary encoding basis as a set of OAM vortex modes. These modes have a top-hat intensity structure and a helical phase profile characterized by $\Psi_{\mathrm{OAM}}^{\ell}=e^{i \ell \varphi}$, where $\ell \in\{-3: 3\}$ (See Fig. 5.1). We construct a mutually unbiased basis set using a linear combination of OAM modes of index $|\ell| \leq N$ with equal amplitudes

$$
\Psi_{\mathrm{ANG}}^{n}=\frac{1}{\sqrt{d}} \sum_{\ell=-N}^{N} \Psi_{\mathrm{OAM}}^{\ell} \exp \left(\frac{i 2 \pi n \ell}{d}\right)
$$

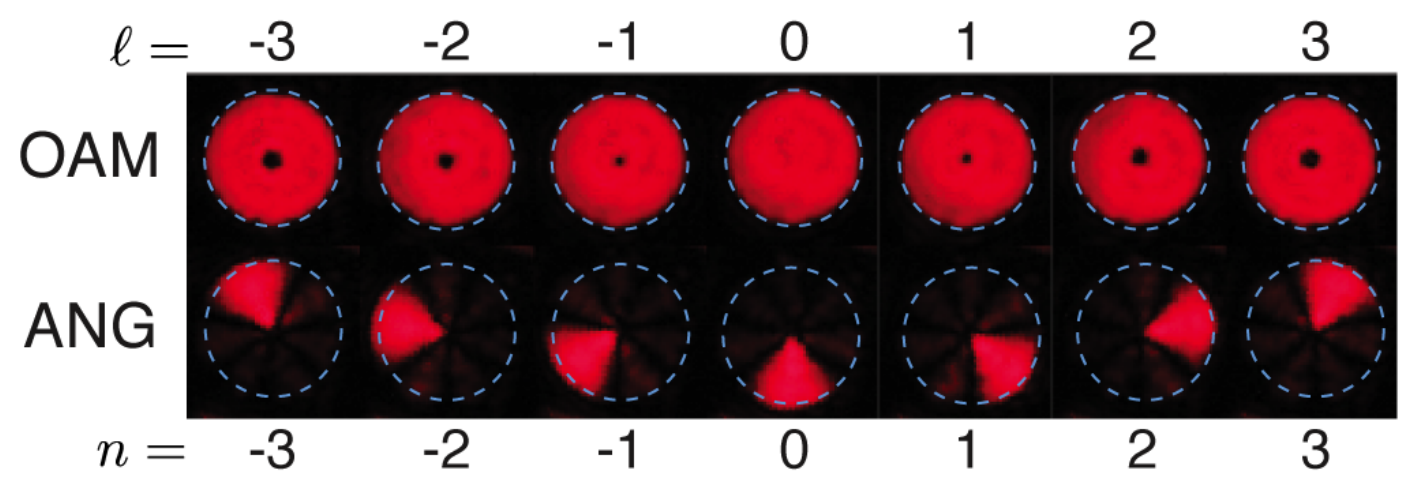

Figure 3.6: The experimentally measured intensity profile of OAM and ANG modes for the case of $d=7$.

where the Hilbert space dimension is set as $d=2 N+1$. We refer to these modes as the angular (ANG) modes due to their localized intensity patterns (See Fig. 5.1). The ANG modes form a mutually unbiased basis with respect to the OAM basis $[84,85,82]$

$$
\left|\left\langle\Psi_{\mathrm{ANG}}^{n} \mid \Psi_{\mathrm{OAM}}^{\ell}\right\rangle\right|^{2}=1 / d \quad \forall\{n, \ell\}
$$

Consequently, the measurement of a photon in the ANG basis provides no information about its OAM state and vice versa. Moreover, the ANG and the OAM bases remain mutually unbiased upon propagation (See Appendix A). In 
our protocol, Alice and Bob randomly choose between the OAM and the ANG modes. In each basis, there exists $d$ independent modes representing different symbols, and hence it is theoretically possible to encode $\log _{2}(d)$ bits of information per each sifted photon. We go over the details of our protocol along with the experimental implementation in the remainder of this chapter. 


\subsection{Generation of OAM and ANG modes}

An OAM mode can be created by simply imposing an $e^{i \ell \phi}$ phase structure onto a laser beam. This task can be achieved by using computer generated holograms [39], q-plates [20], or spiral phase plates [21]. Generation of a secure keys in a QKD system, however, requires two or more mutually unbiased bases (MUBs) [49]. Construction of a MUB for the basis of OAM modes requires shaping both the phase and the amplitude of a laser beam [82, 2]. Phase-only spatial light modulators (SLMs) are widely used for creating such modes [86]. Nevertheless, the vast majority of commercially available SLMs are limited by a frame refreshrate of about $60 \mathrm{~Hz}$ which considerably limits the speed of operation of any system based on this technology.

A Digital Micro-mirror Device (DMD) is an amplitude-only spatial light modulator. The high speed, wide range of operational spectral band-width, and high power threshold of a DMD make it a very useful tool for a variety of applicationsfrom 3D computational imaging [87] to optical control of neuronal activity [88]. Further, variations of DMDs are commercially available for a fraction of the cost of a phase-only SLM. Recently, a DMD was used to encode a varying phase structure onto a beam [89, 90]. The amplitude shaping of spatial modes has been previously achieved by switching the micro-mirrors on and off rapidly [91]. However, the spatial coherence of the generated optical fields is compromised in this process and the created modes have the desired intensity profile only when averaged by a slow detector. In this section, we introduce a method for encoding both phase and amplitude information onto a single binary amplitude mask. By realizing such holograms on a DMD, we have successfully created LG modes, OAM vortex modes, and angular (ANG) modes which form a MUB for the OAM basis. Furthermore, we have directly demonstrated active switching of the generated modes at a speed as high as $4 \mathrm{kHz}$.

To introduce our technique, we consider a one-dimensional binary amplitude grating. The transmission function for this grating can be written as

$$
T(x)=\sum_{m=-\infty}^{\infty} \operatorname{rect}\left[\frac{x-(m+p) x_{0}}{w\left(x_{0} / 2\right)}\right]
$$


(a)

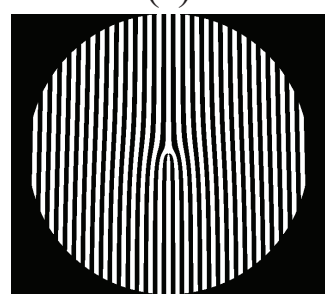

(b)

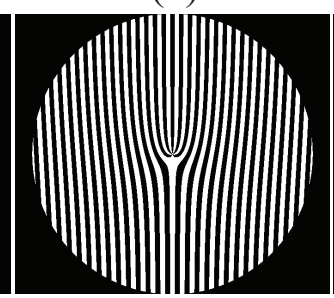

(c)

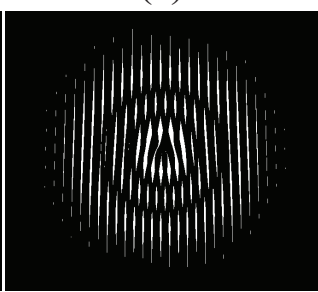

(d)

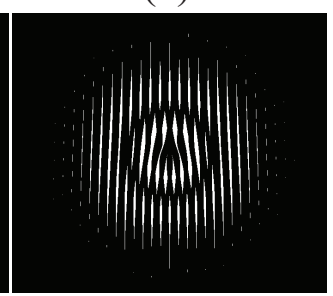

Figure 3.7: Binary hologram for generating (a) $\ell=2$ vortex OAM mode, (b) $\ell=-5$ vortex OAM mode, (c) $L G_{22}$ and (d) $L G_{21}$.

where

$$
\operatorname{rect}(u)= \begin{cases}1 & \text { if }|u| \leq 1 / 2, \\ 0 & \text { else. }\end{cases}
$$

This function can be pictured as a pulse train with a period of $x_{0}$. The parameters $p$ and $w$ are unitless quantities that set the position and the width of each pulse and are equal to constant values for a uniform grating. Here we show that it is possible to locally change the value of these parameters to achieve phase and amplitude modulation of the optical field.

The transmittance function $T(x)$ is a periodic function and can be expanded as a Fourier series as

$$
T(x)=\sum_{m=-\infty}^{\infty} T_{m} \exp \left[i 2 \pi m\left(\frac{x}{x_{0}}\right)\right] .
$$

The coefficients $T_{m}$ are a functions of parameters $w$ and $p$ and are given by

$$
T_{m}=\frac{\sin \left(\frac{\pi m w}{2}\right)}{\pi m} e^{i 2 \pi m p}
$$

where we have assumed that both $w$ and $p$ are bounded between zero and one. Now, we consider a case where $p(x)$ and $w(x)$ are functions of $x$ and the binary grating is illuminated by a monochromatic plane wave. The first-order diffracted light can be written as

$$
T_{1}(x)=\frac{1}{\pi} \sin \left[\frac{\pi w(x)}{2}\right] e^{i 2 \pi p(x)}
$$




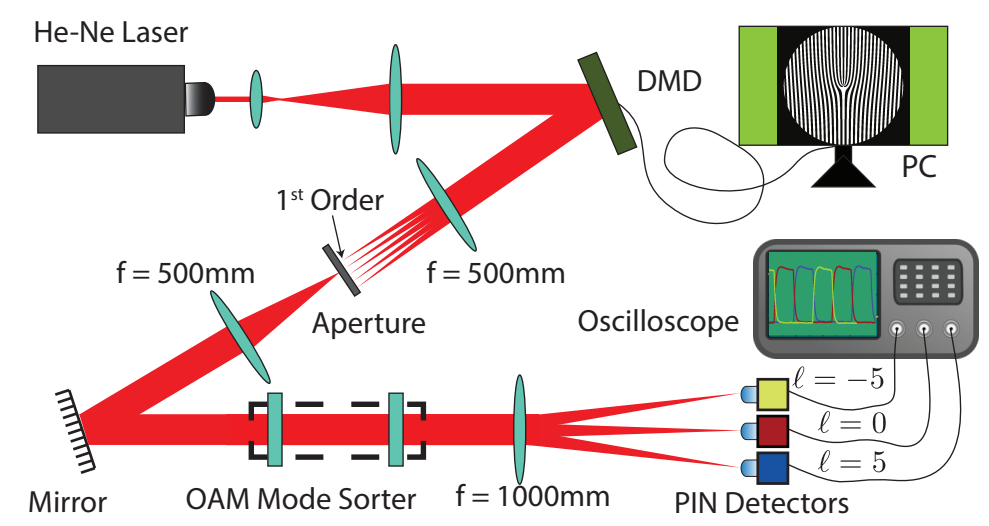

Figure 3.8: Schematic diagram of the experimental setup for a) measuring intensity profile and phase interferograms. b) switching among three OAM modes and detecting them in real time.

It can be seen that $w(x)$ is related to the amplitude of the diffracted light while $p(x)$ sets its phase. Therefore, the phase and the amplitude of the diffracted light can be controlled by setting the parameters $p(x)$ and $w(x)$. In the language of communication theory, these methods are sometime referred to as pulse position modulation (PPM) and pulse width modulation (PWM) [92, 93]. Eq. 3.4.5 provides an accurate approximation for slowly varying $p(x)$ and $w(x)$ functions $[94,95]$, when a mixed Fourier-Taylor analysis can be used to derive this formula [96].

The analysis above treats a one-dimensional case. To encode phase and amplitude in two dimensions, we have generated the pulse sequence by thresholding a rapidly varying modulated carrier

$$
T(x, y)=1 / 2+1 / 2 \operatorname{sgn}\left(\cos \left[2 \pi x / x_{0}+\pi p(x, y)\right]-\cos [\pi w(x, y)]\right) .
$$

Here, $\operatorname{sgn}(x, y)$ is the sign function. It is easy to check that in in the limit where $w(x, y)$ and $p(x, y)$ are slowly varying, this formula reproduces the pulse train described above. We can find the corresponding $w(x, y)$ and $p(x, y)$ functions for a general complex scalar field $\mathcal{A}(x, y) e^{i \varphi(x, y)}$ according to the relations

$$
w(x, y)=\frac{1}{\pi} \arcsin [\mathcal{A}(x, y)]
$$




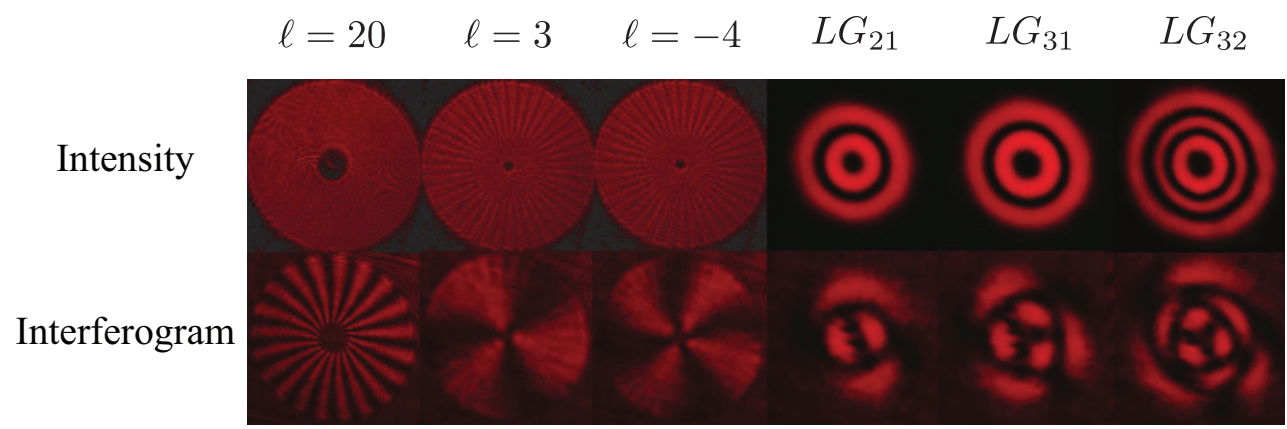

Figure 3.9: Intensity and interferograms of three vortex modes (to the left) and LG modes (to the right). The interferograms demonstrate the phase structure of the beams and have been obtained by interfering the generated modes with a plane wave.

$$
p(x, y)=\frac{1}{2 \pi} \varphi(x, y) .
$$

We have assumed the amplitude contains no singularity and thus its maximum can be normalized to the value of unity.

We have designed two-dimensional binary amplitude holograms to generate Laguerre-Gaussian (LG) modes. Fig. (3.7) shows sample holograms designed for generation of vortex OAM and LG modes. It can be seen that in both cases, the holograms have the familiar forked structure. The gratings designed for vortex modes have a fairly uniform width across the aperture whereas for the case of LG modes the gratings gradually disappear where the amplitude gets negligibly small.

A digital micro-mirror device (DMD) is an amplitude-only spatial light modulator. The device consists of a series of micro-mirrors that can be controlled in a binary fashion by setting the deflection angle of each individual mirror to either +12 or -12 degrees. This enables the on-demand realization of binary gratings that can be switched at very high speeds using an external digital signal [97].

In our experiment, we generate spatial modes by loading computer generated holograms described in the previous section onto a DMD. We have used a Texas Instrument DLP3000 DMD for this task. As shown in Fig. (5.1), a $4 \mathrm{f}$ imaging system along with an aperture separates the first order diffracted light. We use a charge-coupled-device (CCD) camera for measuring the intensity profile of 


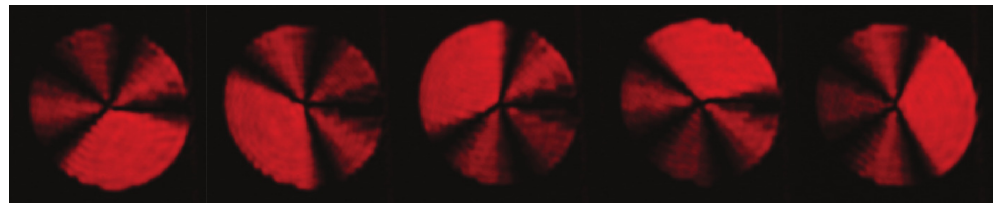

Figure 3.10: Intensity patterns for ANG modes constructed from superposition of vortex OAM modes from the set $\ell \in[-2: 2]$.

the generated modes. In addition, we have used a Mach-Zehnder interferometer for verifying the phase patterns of the created beams. A collimated plane-wave reference from the same laser is interfered with the modes generated by the DMD to obtains interferograms.

We have created OAM modes and LG modes using the setup. Fig. (3.9) shows the intensity pattern along with the interferogram for a few of these modes. The structure of the interferograms is in agreement with the helical phase pattern of OAM modes, with the number of azimuthal dark lines equating to the OAM quantum number in each case. The LG modes have the same azimuthal phase structure as the vortex modes. In addition, these modes contain $p+1$ rings in the radial direction where $p$ is the radial quantum number [14]. The change of sign of the optical field between each two consecutive rings is an intrinsic property of the LG modes and it can be clearly noticed in the interferograms.

The examples above demonstrate the possibility of using binary holograms to coherently control both phase and amplitude of a beam. Although the OAM modes can be generated by only shaping the phase, creating the ANG modes requires the ability to shape both the intensity and the phase. We have generated ANG modes using our technique. The amplitude profile of a number of these modes is presented in Fig. (3.10).

Using a DMD for generating OAM modes gives us the ability to switch between different modes at very high speeds. This method involves a much smaller number of optical elements as compared to the conventional techniques where OAM modes are generated using a series of separated forked holograms and are multiplexed using beam splitters [37]. As a direct test of the highspeed capability of our mode-generation system, we have implemented dynamic switching among vortex OAM modes with quantum number $\ell=5,-5$ and 


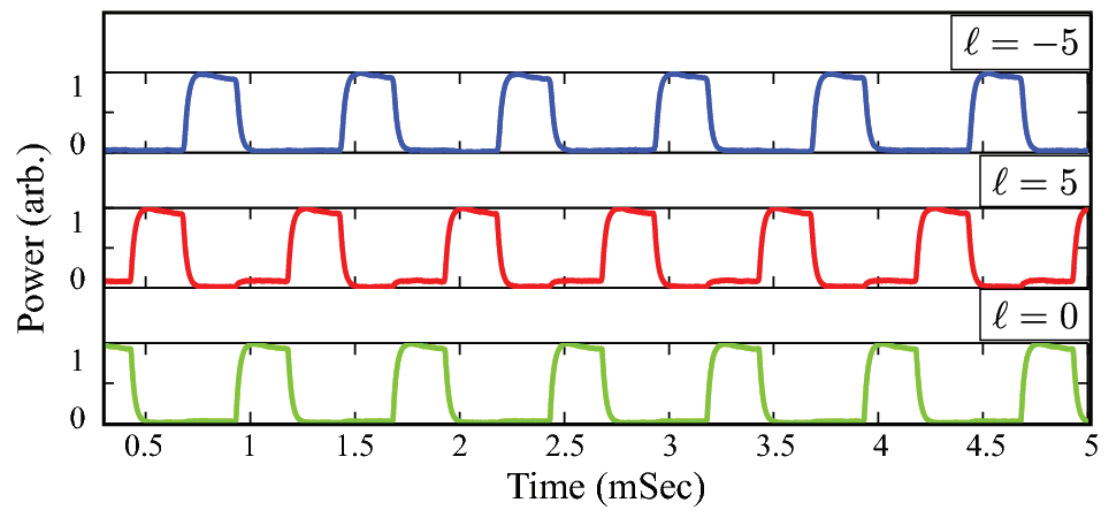

Figure 3.11: Detected power as function of time for three vortex OAM modes of $\ell=5, \ell=-5$ and $\ell=0$. It is seen that the generated mode can be changed on a time-scale of $0.25 \mathrm{~ms}$ (measured at FWHM) corresponding to a switching rate of $4 \mathrm{kHz}$.

0. The computer generated holograms for these modes were loaded onto the memory of the DMD and the switching was achieved by using a clock signal. We have used the mode sorter described in [4] to map the input modes to a series of separated spots. We then measured the intensity corresponding to each mode using a high bandwidth PIN detector at the positions corresponding to each mode. Fig. (3.9) demonstrates the measured values. It can be seen that the generated OAM quantum number can be controlled with a speed of $4 \mathrm{kHz}$. This is a clear demonstration of the capability to rapidly switch among such modes. It should be noted that the speed reported above is a limitation imposed by the DMD we have used and it is not inherent to this technique. In fact, commercially available DMDs can achieve a speed of as high as $32 \mathrm{kHz}$ (TI DLP7000). Further, these devices are available for a fraction of the cost of a phase-only spatial light modulator. 


\subsection{Experimental realization}

In this section we describe a proof-of-principle experiment that demonstrates the feasibility of performing high-dimensional quantum cryptography with OAM modes. We encode information in a 7-dimensional set of OAM modes along with modes in the mutually unbiased basis of azimuthal angle (ANG). Our scheme uses a digital micro-mirror device (DMD) for fast generation, and an efficient technique for unambiguous sorting of both OAM and ANG modes. By combining these techniques, we selectively generate the set of 14 spatial modes at a rate of $4 \mathrm{kHz}$ and correspondingly detect them with a separation efficiency of $93 \%$. We measure a channel capacity of 2.05 bits per sifted photon with an average symbol error rate of $10.5 \%$ that is well below the error bounds that are required for security against intercept-resend eavesdropping attacks. Of our symbol error rate, $4 \%$ is due to detector dark counts and ambient light, and $6.5 \%$ is due to errors due to intermodal crosstalk.

Our experimental setup is depicted in Fig. 5.1. A collimated beam from a helium-neon laser illuminates a binary hologram realized on a DMD to generate spatial modes [6]. We use a prepare-and-measure scheme similar to the BB84 protocol [49]. Alice initially picks a random sequence of desired OAM and ANG modes and loads the binary holograms for generating each of the modes in the sequence to the DMD's internal memory. To transmit each symbol, Alice then triggers the DMD and modulates the beam using an acousto-optic modulator to create rectangular pulses of $125 \mathrm{~ns}$ width. The beam power is attenuated such that each pulse contains on average $\mu=0.1$ photons. The prepared states are then imaged to Bob's receiving aperture via a $4 f$ telescope that forms a lossless 2-m-long communication link.

Bob's mode sorter consists of two refractive elements for performing a logpolar to cartesian transformation [4]. Going through these elements, an OAM mode is converted to a plane wave with a tilt that is proportional to the OAM mode index $\ell$. A single lens focuses such a plane wave into a spot that is shifted by an amount proportional to $\ell$. Similarly, an ANG mode transforms to a localized spot shifted by an amount proportional to the angular index $n$ after 


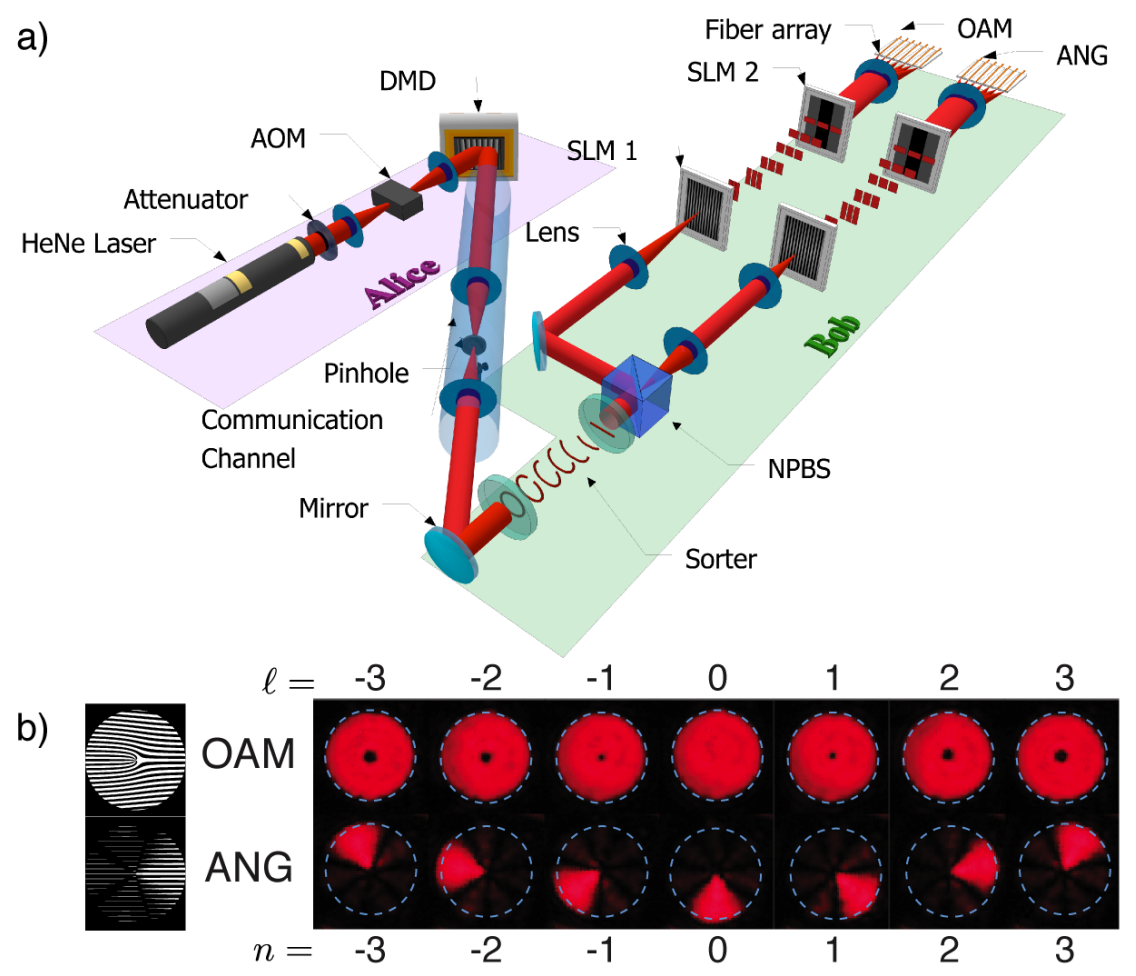

Figure 3.12: a) The experimental setup. Alice prepares the modes by carving out pulses from a highly attenuated He-Ne laser using an AOM. The spatial mode information is impressed on these pulses with a DMD. Bob's mode sorter and fan-out elements map the OAM modes and the ANG modes into separated spots that are collected by an array of fibers. b) The alphabet. CCD images of produced light field profiles in two complementary spatial bases of OAM and ANG $(\mathrm{d}=7)$. The intensity profile of the modes are shown on the right. An example of binary holograms used for the generation of modes in each basis is shown on the left.

the transformation. A beam splitter is used to randomly choose between the OAM and ANG bases. In order to reduce the overlap between the neighboring transformed modes, we use a coherent beam combination technique described in the previous chapter [1]. A fan-out element is realized on a phase-only spatial light modulator (SLM) to divide the incident light into multiple copies. The copies are phase corrected using a second SLM and are recombined by a lens to a series of much narrower spots with the same initial spacing between them. In addition, two cylindrical lenses are realized on the SLMs to adjust the aspect ratio of the transformed modes. 
a)

Alice

\begin{tabular}{|l|}
\hline 063133602132045444456141026645 \\
545050363603025261643215524164 \\
230146602513401613222451551026 \\
\hline
\end{tabular}
Bob

$063 \underline{0} 336011320 \underline{21} 444456141026645$
$5450 \underline{22} 3 \underline{5} 3603025261643215524164$

$23014660251 \underline{5} 40 \underline{3} 613 \underline{4} 22451551026$

\section{Error Correction}

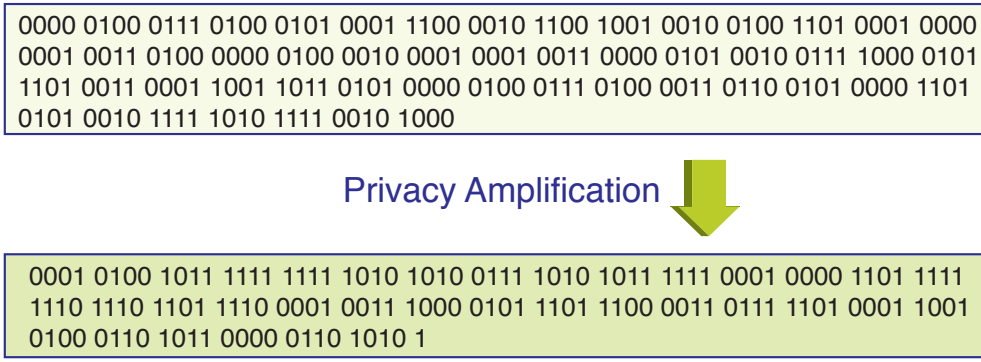

Privacy Amplification

000101001011111111111010101001111010101111110001000011011111 111011101101111000010011100001011101110000110111110100011001 0100011010110000011010101

b)

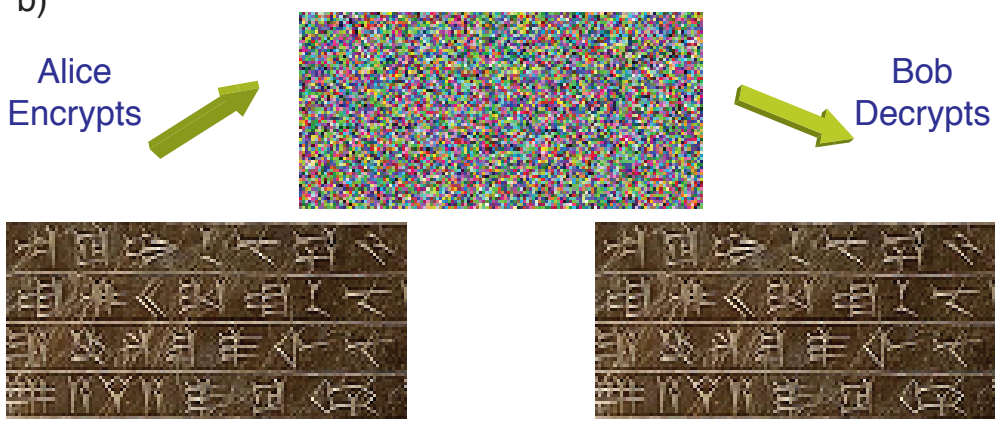

Figure 3.13: Key generation a) An example of a random sifted key from the experiment. The spatial modes are mapped to number between 0 to 6 (errors are marked in red and underlined). Each symbol is converted into a 3 digit binary number first and the binary key is randomized before the error-correction. Privacy amplification minimizes Eve's information by shortening the key length. b) Alice encrypts the secret message (a picture [?]) using the shared secure key and Bob decrypts it. In this case a short key is repeated many times to match the bit length of the image. In practice, multiple use of a short key cannot provide security and a long key needs to be used.

The sorted modes are coupled to an array of fibers that are connected to avalanche photodiodes (APDs). Due to the limited number of available APDs (four), Bob's data for each group of the the symbols is taken separately and is combined later. The signal from the APDs is processed by a field-programmable gate array (FPGA) to produce photon counts using a gating signal shared with Alice. The photon detection events are finally saved in Bob's computer. Alice and Bob are also connected via a classical link realized by an ethernet cable 
running a TCP/IP protocol. After Alice and Bob collect a sufficiently large number of symbols, they stop the measurement. At this point, they publicly broadcast the bases used for preparation and measurement of each photon via the classical link. Alice and Bob then discard the measurements that were done in different bases. The key generated at this stage is referred to as the sifted key.

Figure 3.13 shows a portion of the sifted key generated in the experiment. At this stage, the two copies of the key owned by Alice and Bob are almost identical but they contain some discrepancies. The discrepancies are due to the imperfections in our system, namely imperfect mode-sorting, detector dark counts, and ambient light. However, any errors in the key must be attributed to an eavesdropper in the security analysis. The keys are transformed to a binary form on a symbol-by-symbol basis and randomized by means of a random-number generator shared by Alice and Bob. The cascade error-correction protocol is run by Alice and Bob to fix the discrepancies by comparing the two keys in a blockby-block fashion and performing parity checks [98]. After error-correction, Alice and Bob share two identical copies of the key. However, a portion of the key can still be known to Eve from eavesdropping on the quantum channel or from accessing the publicly available information transmitted in the classical channel during the error correction. Alice and Bob perform privacy amplification to minimize Eve's information. This is done by using a universal hash function for mapping the (error-corrected) key to a shorter, more secure random key [99]. The amount by which the key is shortened at this stage is set by the estimated amount of information that can be owned by Eve. Finally, the secure key is used to securely transmit information (a picture in this case) over the classical channel (See Fig. 3.13).

\section{Characterization of the transmission and detection efficiencies}

The power transmission efficiency of the mode sorter's refractive elements is measured to be $85 \%$. Two Holoeye PLUTO phase-only SLMs are used for realizing the holograms for the fan-out element and its corresponding phase-correcting element. In addition, two cylindrical lenses are realized on the SLMs to adjust 
(a)
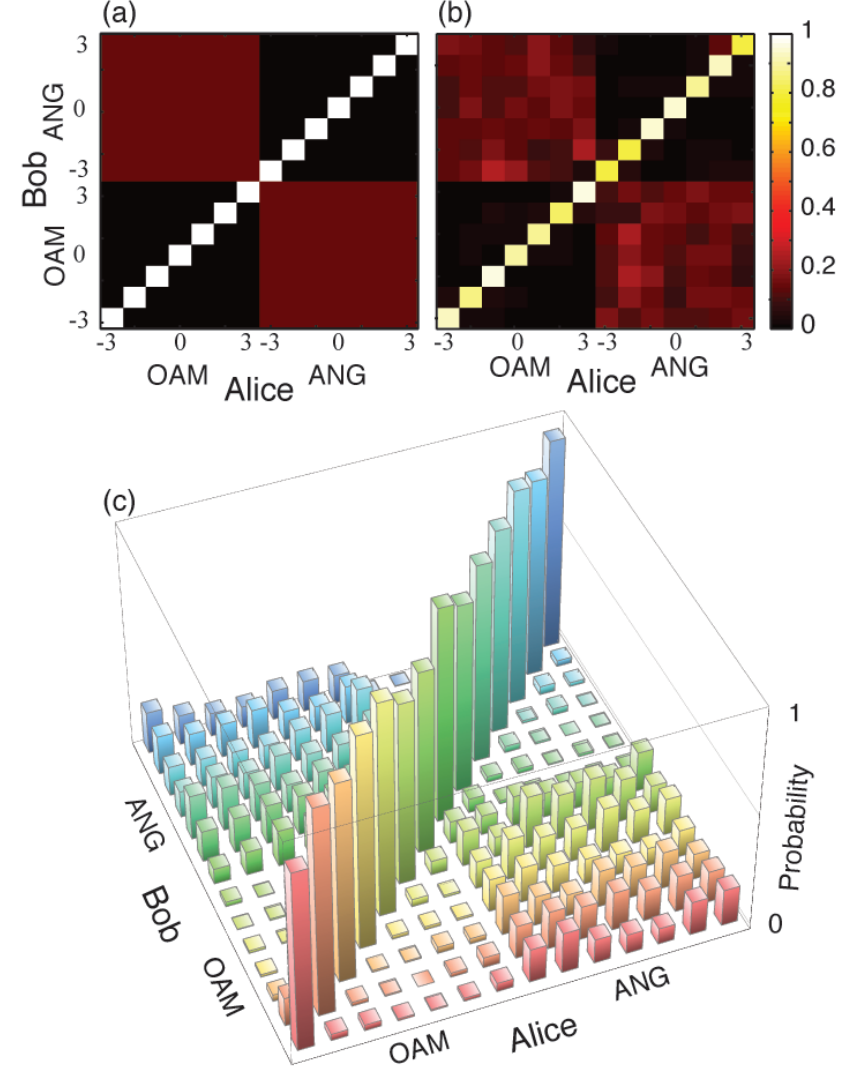

Figure 3.14: Conditional probability of detection. a) Theoretical predictions for an ideal system. The bases chosen by Alice and Bob are marked on the horizontal axis. b)The experimental results. To construct the matrix a total number of 14 million pulses with $\mu=0.1$ photon per each are sent by Alice. The signals from APDs and the gate are collected using a $300 \mathrm{MHz}$ oscilloscope on the fast acquisition mode and processed on a computer. c) 3D view of the experimental data.

the aspect ratio of the transformed beams. The diffraction efficiency of each SLM is measured to be approximately $45 \%$.

The fiber array consists of 7 multi-mode fibers with $62.5 \mu \mathrm{m} / 125 \mu \mathrm{m}$ core/cladding diameters and $\mathrm{NA}=0.275$. We have measured the efficiency of coupling the transformed modes to the fiber array to be approximately $18 \%$. The detection is performed with Perkin-Elmer SPCM-AQRH-14 APDs. The quantum efficiency of the APDs is $\eta=0.65$. The transmission efficiency of the optical link can be 
calculated as

$$
T_{\text {link }}=0.85 \times(0.45)^{2} \times 0.18=0.031 .
$$

Each detectors has a typical dark-count rate of $50 \mathrm{c} / \mathrm{s}$ and an after-pulsing probability of $0.3 \%$

\section{Calculation of the classical Information capacity}

Figure 3.14 shows the conditional probability of Bob detecting each mode as a function of the mode sent by Alice. Theoretical values for the case of a system with no errors is shown on the left for comparison. In order to measure the symbol error rate more precisely, each row of the matrix is constructed by sending the same symbol many times and detecting all possible outcomes. This scheme eliminates the time consuming procedure of switching among different spatial modes and permits a much more accurate measurement of the error-rate using a large set of sent and received symbols.

The mutual information between the sent and received symbols is defined as

$$
I_{A B}=\sum_{i, j} P\left(y_{j}, x_{i}\right) \log _{2}\left[\frac{P\left(y_{j}, x_{i}\right)}{P\left(x_{i}\right) P\left(y_{j}\right)}\right] .
$$

Here, $x_{i}$ is the event of sending symbol $i$ and $y_{j}$ is the event of detecting symbol $j$. Assuming a uniform probability for sending $N$ modes and a uniform probability of an error occurring in the detection, the Eq. 3.5.2 can be simplified to [11]

$$
I_{A B}=\log _{2}(d)+F \log _{2}(F)+(1-F) \log _{2}\left(\frac{1-F}{d-1}\right) .
$$

Here, $F=1-\delta-\epsilon$ is the probability of correctly detecting each mode. The symbol error rate caused by imperfect sorting is denoted by $\delta$ and the symbol error rate due to the dark counts is shown by $\epsilon$. For the case of our experiment, $\delta=0.065$ and $\epsilon=0.04$. The total symbol error rate $1-F$ is directly measured from data as $10.5 \%$. We have identified the error from the imperfect sorting, $\delta$, by repeating the experiment in the high-light level and analyzing the results after background subtraction. The error probability $\epsilon$ can be divided to $2 \%$ from stray 
light, $1.9 \%$ from thermally-induced dark counts, and $0.15 \%$ from after-pulsing.

Using these numbers, we calculate $I_{A B}$ as 2.05 bits per photon. For comparison, the ideal value of $I_{A B}$ for $d=7$ modes is equal to $\log _{2}(d)=2.8$ bits per photon. Further, the sorting mechanism by itself would result in a value of 2.29 bits per photon in the absence of any background or dark counts. The probability of correctly detecting each mode in the absence of dark counts, also known as the separation efficiency of the mode sorter, is calculated to be slightly more than $93 \%$.

Note that the calculation of mutual information only requires the knowledge of the cross-talk and dark count values. The loss in the optical system reduces the key generation rate of the protocol. However, a uniform loss in the transmission and detection does not change the mutual information between Alice and Bob's sifted keys since the time frames with no photon-detection event are removed in the basis reconciliation procedure.

\section{Calculation of the secure key rate}

We consider a cloning-based individual attack. In this situation, the mutual information between the symbols owned by Alice and Eve can be written as [11]

$$
I_{A E}=\log _{2}(d)+F \log _{2}\left(F_{E}\right)+\left(1-F_{E}\right) \log _{2}\left(\frac{1-F_{E}}{d-1}\right) .
$$

Here, $F_{E}$ is the fidelity of Eve's cloning machine. The value of Eve's fidelity can be optimized to gain maximum information for any given symbol error rate detected by Bob. This quantity is shown to be [11]

$$
F_{E}=\frac{F}{d}+\frac{(d-1)(1-F)}{d}+\frac{2}{d} \sqrt{(d-1) F(1-F)} .
$$

For our experiment, $F_{E}=0.43$, resulting in $I_{A E}=0.35$ bits. For a sufficiently long ensemble of symbols owned by Alice, Bob, and Eve the secure key $R$ is found to be $[100,50]$

$$
R_{\text {net }}=R_{\text {sift }}\left[I_{A B}-\max \left(I_{A E}, I_{B E}\right)\right],
$$


where $R_{\text {sift }}$ is the sifted key rate. This rate can be written as a function of the pulse repetition rate $f_{\text {rep }}$

$$
R_{\mathrm{sift}}=\frac{1}{2} f_{\mathrm{rep}} \mu T_{\mathrm{link}} \eta
$$

For our experiment we have $\mu=0.1$. The DMD used in our system (Texas Instrument DLP3000) is capable of switching between the computer holograms stored in its memory at the speed of $4 \mathrm{kHz}$. We refer to this mode of operation as burst mode. Burst mode can be used to transmit a short key at $f_{\text {rep }}=4$ $\mathrm{kHz}$. The internal memory can only store up to 100 patterns, which limits the applicability of the burst mode for a long key. To generate a long random key, we instead load each hologram onto the DMD using a computer. This task along with the time needed for synchronizing the two computers reduces the raw key generation rate to approximately $f_{\text {rep }}=1 \mathrm{~Hz}$. Using $f_{\text {rep }}=4 \mathrm{kHz}$ for the burst mode, we calculate $R_{\text {sift }}=4$ (photons $/ \mathrm{sec}$ ) and $R_{\text {net }}=6.8$ (secure bits $/ \mathrm{sec}$ ). Note that this calculation characterizes the operation of the optical system in the presence of 14 detectors for collecting data.

The calculation above can be recast to the alternative form

$$
R_{\text {net }}=f_{\text {rep }} I_{\text {pulse }}
$$

Here, $I_{\text {pulse }}$ is the amount of secure information transmitted per each pulse. For our experiment we can infer $I_{\text {pulse }}=1.7 \times 10^{-3}$ secure bits per pulse.

To quantitatively assess the security of our system, we plot the values for the error bound of an intercept-resend eavesdropping attack, a coherent attack, as well as the symbol error rate from our experimental data in Fig. 3.15. In an intercept-resend attack, the eavesdropper (Eve) measures the state of the intercepted photon in an arbitrarily selected basis and then resends a photon prepared to be in this same state. In a coherent attack, Eve coherently probes a finite number of qudits in order to gain information about the key [11]. It is evident from this graph that our experimental symbol error rate is well below the required bounds for security against both intercept-resend and coherent attacks (Fig 3.15). Unlike intercept-resend attacks, the symbol error rate for coherent attacks only depends on system dimension $d$ and is independent of the number 


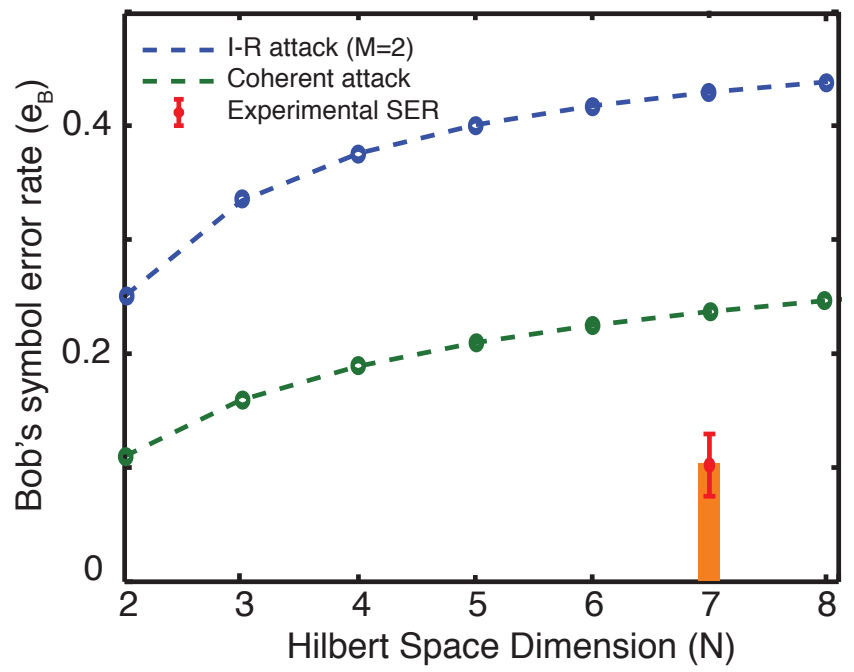

Figure 3.15: Error-bound for security. Bob's error bound calculated from theory as a function of the dimension of the Hilbert space for intercept-resend attacks with two MUBs $(\mathrm{M}=2)$ and coherent attacks is plotted along with symbol error rate measured from our experimental data (the error bar shows one standard deviation). It is seen that the symbol error rate from our experiment lies well below the theoretical bounds and hence it is sufficient for proof of security.

of MUBs $M$ [11]. Hence, increasing the number of MUBs beyond 2 results in a drop in the key rate without any gain in security against coherent attacks. Nevertheless, there is a clear increase in the allowed symbol error rate for larger system dimensions, demonstrating an advantage for using a high-dimensional encoding scheme for QKD such as that of OAM.

Using the symbol error rate, we estimate the information gained by Eve to be 0.35 bits per sifted photon for cloning-based individual attacks. It has been argued that cloning-based eavesdropping is the optimal strategy for an individual attack on systems based on qudits [11]. The portion of the key obtained by Eve is removed in the privacy amplification process, leading to a mutual information of 1.7 secure bits per sifted photon in the final key. It should be emphasized that our secure key rate analysis assumes an infinite key, while our experimental realization produces only a finite key [101]. We are working toward a finite key analysis of the security, which will be reported in the future.

In contrast to phase-only spatial light modulators (SLMs) that are limited to a frame rate of $60 \mathrm{~Hz}$, the DMD used in our setup has the ability to rapidly switch 
between modes at a speed of $4 \mathrm{kHz}$. This is especially important considering the fact that the speed of generation of spatial modes limits the key generation rate of the system. We measure our raw key generation rate to be 16.4 bits per second (for operation in burst mode). After performing basis reconciliation and privacy amplification for a sufficiently long key, we estimate the secure key rate to be 6.5 bits per second, which is more than 3 orders of magnitude larger than that achieved in previous spatial-mode based protocols [68].

\section{Photon number splitting attacks}

The analysis above considers intercept-resend and cloning based attacks. The photon-number-splitting (PNS) attack is yet another possibility when the protocol uses imperfect single-photon sources. In our protocol, weak coherent pulses (WCPs) are used for realizing approximate single photon pulses. For small values of photon-number expectation values, a WCP contains either zero or one photon most of the times. However, the pulse may still contain multiple photons with a non-zero probability. In this situation, Eve can split one photon off a multiphoton signal, without disturbing the polarization or the spatial structure of the signal $[102,103,104]$. Eve can measure her photon after basis reconciliation and obtain complete information without causing any error in the signal.

The PNS attacks pose a more serious threat in the presence of loss. In this situation, Eve can, in principle, replace the lossy channel with a perfect quantum channel and only transmit to Bob the signals of her choice. An inequality has been developed in [105] that serves as a necessary condition for security

$$
p_{\text {detection }}>p_{\text {multi }}
$$

Here, $p_{\text {detection }}$ is the total probability of detection events for Bob, and $p_{\text {multi }}$ is the probability of having a pulse with multi-photons. For a coherent state with an average photon number of $\mu$, we have $P(n, \mu)=\frac{\mu^{n}}{n !} e^{-\mu}$, and consequently

$$
p_{\text {multi }}=\sum_{n=2}^{\infty} P(n, \mu) \approx \frac{\mu^{2}}{2} .
$$


The total probability of detection events can be calculated by considering the signal detection events and the detector dark counts

$$
p_{\text {detection }}=p_{\text {signal }}+p_{\text {dark }}-p_{\text {signal }} p_{\text {dark }} \approx p_{\text {signal }}+p_{\text {dark }}
$$

Using the parameters for our experiments we have $p_{\text {multi }}=5 \times 10^{-3}$ and $p_{\text {detection }}=$ $\mu T_{\text {link }} \eta+p_{\text {dark }} \simeq 2 \times 10^{-3}$. It can be see that $p_{\text {detection }}<p_{\text {multi }}$ and hence security against PNS attacks cannot be guaranteed in our current implementation. We discuss the possible approaches for providing security against PNS attacks in the following section.

\section{Effect of imperfect MUBs}

The unbiased relation between the two bases, as defined in Eq. 3.3.5, guarantees that detecting a photon in the wrong basis results in a completely random outcome and hence it reveals zero information about the key. In practice, however, the imperfect generation and sorting of the OAM and ANG modes results in variations in the degree of overlap between a pair of modes chosen from the two different bases. Such variations can be noticed in the off-diagonal blocks in the matrix for conditional probability of detection (Fig. 3.14). In this situation, the measurement of a photon in the wrong basis can, in principle, reveal some information about the transmitted symbol.

The mutual information between the symbols sent in the OAM basis and detected in the ANG basis can be calculated using Eq. 4. For our experiment, we have calculated $I_{\mathrm{OAM} / \mathrm{ANG}}=0.036$ bits per photon. Similarly, the mutual information for the case of sending a photon in the ANG basis and detecting it in the OAM basis is calculated to be $I_{\mathrm{ANG} / \mathrm{OAM}}=0.058$ bits per photon. Note that these values are much smaller than $I_{A B}$ which is equal to 2.05 bits per photon. We have assumed an unbiased relation between the OAM and ANG modes in our security analysis. The small number the quantities $I_{\mathrm{ANG} / \mathrm{OAM}}$ and $I_{\mathrm{OAM} / \mathrm{ANG}}$ calculated above justifies the validity of this approximation. A more complete security analysis needs to take these numbers into account in estimating the information owned by Alice $\left(I_{A E}\right)$. Such an analysis, however, is beyond the 
scope of current article and should be the subject of future studies. 


\subsection{Steps towards practical QKD with OAM modes}

The secure key rate of a QKD system is considered as the ultimate metric for quantifying its performance. In the above sections we described how a highdimensional encoding scheme such as that of OAM can potentially benefit the secure key rate of QKD protocols. While our experiment provides significant improvements in realizing OAM-based QKD, several challenges need to be addressed before such a protocol can be employed for practical applications. In addition to a high secure key rate, a free-space QKD link needs to provide reliable long distance operation, efficient classical post-processing, ease of alignment, and a rigorous security analysis considering the finite size of practical keys. While some of these requirements can be achieved by increasing the degree of sophistication of the system or through the use of state-of-the-art technology, there is still need for theoretical and experimental research before a full demonstration can be performed. Below, we address some of the current limitations.

Fast key generation. The secure key rate scales proportionally with the raw key generation rate. A fast rate can be achieved by using an array of static holograms for generating multiple modes and then multiplexing the modes using a series of beam splitters. A cheaper and much simpler alternative to this approach is to modulate the intensity and the phase of a laser beam in real time. Although holography techniques can achieve $\mathrm{kHz}$ mode switching rates using DMDs [6], practical QKD would require key rates in the $\mathrm{GHz}$ regime. OAM modes can be generated and switched at $\mathrm{MHz}$ rates using on-chip resonators [106] and potentially at $\mathrm{GHz}$ rates using Q-plates [107]. However, to generate the states in the ANG basis one would need to modulate both the amplitude and phase of the beam, a task that best suits free-space holography. Recently, a method involving static holograms realized on an SLM and an AOM for switching between them achieved a $\mathrm{MHz}$ mode-switching rate [108]. Unfortunately, the change in the wavelength caused by the AOM forbids applying this method for QKS since side-channel attacks could be performed based on spectrum.

Alternatively, generation of OAM and ANG modes at a rate of $1 \mathrm{GHz}$ or 
more can be achieved by illuminating a series of static holograms with beams from multiple lasers. The shaped beams after the holograms can be passively combined using a series of beam splitters. The intensity of each laser beam can then be modulated either by using electro-optic modulators or by switching diode lasers on and off [37].

High throughput. The key generation rate drops as loss increases. Moreover, high loss in the communication link makes the protocol vulnerable to PNS attacks. The throughput of our detection system can be readily increased by employing high efficiency spatial light modulators, or AR-coated custom refractive elements. This would translate to a six-fold increase in the transmission efficiency in our experiment. Additionally, the amount of loss due to the scattering in the air can be minimized by operating in the near-infrared regime.

Turbulence mitigation. Atmospheric Turbulence results in degradation of the spatial profile of the modes upon propagation. This results in mixing of the neighboring OAM and ANG modes. The effects of turbulence on OAM modes has been a topic of extensive studies [82, 78, 77]. Common solutions for mitigating the adverse effects of atmospheric turbulence include the use of every other mode for encoding [80] and utilization of adaptive optics systems [79]. Recently, long-haul free-space propagation of OAM modes has been realized using novel detection schemes [76].

Larger dimensionality. Increasing the number of the modes increases the information carried by each single photon and results in a higher secure bit rate. Previously, we have shown that our mode sorter is capable of sorting 25 OAM and ANG modes with an average mutual information of 4.17 bits per detected photons [1]. Consequently, the encoded information per photon can be readily increased by increasing the number of APDs in the experiment. Ultimately, the number of modes supported by the optical link is limited by the sizes of the transmitting and receiving apertures.

Universal security proof. A comprehensive security proof for a QKD system should include both the fundamental and practical properties of the physical system. The security analysis presented in this work assumes an infinitely long key. For a finite key, the efficiency of classical post-processing need to be measured 
and used for a more rigorous calculation of the secure key rate [109]. In addition, our analysis assumes a uniform error-rate and transmission efficiency for all the modes. While our data demonstrates the rationale for this approximation, a more comprehensive analysis needs to include the effects of non-uniform symbol error rate and loss in a multi-level QKD system. More research is needed to establish the theoretical framework for security analysis of such systems.

Finally, we have demonstrated security against intercept-resend and cloningbased attacks, while our implementation remains vulnerable to photon-numbersplitting attacks. This limitation can be avoided by either reducing the loss in the system or by employing the decoy-state protocol [110] 


\subsection{Summary}

In this chapter we have demonstrated that a functional QKD system based on spatial-mode encoding is realizable with current technology. We have provided a brief review of the BB-84 quantum cryptography and then generalized it to multidimensional systems. Our analysis of free-space communication with spatial modes justifies the choice of OAM modes for information encoding in multilevel line-of-sight QKD scheme. We have reported a proof-of-principle experiment that uses a 7-dimensional alphabet encoded in OAM and in the mutual unbiased basis of ANG modes. We have implemented a fast mode-generation technique that uses a DMD to generate spatial modes at a speed of $4 \mathrm{kHz}$ and a mode-transformation technique that is able to measure the OAM and ANG content of a photon with an accuracy of $93 \%$. Using these two methods, we have achieved a mutual information of 2.05 bits per sifted photon, which is more than twice the maximum allowable capacity of a two-dimensional QKD system. The symbol error rate of our scheme is measured to be $10.5 \%$, which is sufficient for proving security against coherent and individual eavesdropping attacks for an infinite key. In addition, we have layed out a clear path for how our system can be enhanced to perform practical, high-dimensional QKD using current technology. For example, our symbol error rate can be significantly reduced through the use of better detectors and a more sophisticated mode-transformation technique. 


\section{Chapter 4}

\section{Direct measurement of the wave function}

\subsection{Introduction}

The no-cloning theorem prohibits exact determination of the quantum wave function from a single measurement $[51,52,111]$. In contrast, a large ensemble of identically prepared quanta can be used to estimate the wave function through quantum state tomography (QST) $[112,113,114,115,116,117,118,119,120]$. QST requires the measurement of $d^{2}-1$ independent real numbers for characterizing a $d$-dimensional system. The rapid growth in the number of the requited measurements along with the time-consuming post-processing make QST increasingly more challenging as $d$ grows $[117,121]$.

Recently, there has been considerable interest in determining the wave function of a pure quantum state through the use of weak values $[10,122,9,123$, 124, 125]. This method, known as the direct measurement, provides a convenient procedure for estimation of a wave function. It has been suggested that direct measurement (DM) is an efficient means of characterizing high-dimensional states due to the simplicity of its realization and absence of time-consuming post processing [10]. Direct measurement is based on the idea that weak value is a

complex quantity, and hence the real and imaginary parts of the wave function 
can be retrieved at once if the wave function can be linked to a weak value. This is achieved by a sequence of weak and strong measurements, performed in the position and momentum bases respectively. We begin this chapter by providing a brief review of the theory of direct measurement.

We have applied direct measurement to the the problem of characterization of a superposition of OAM modes. By performing a weak measurement of orbital angular momentum (OAM), followed by a strong measurement of azimuthal angle, we find the quantum state in the Hilbert space spanned by 27 OAM modes. In addition, we use this method to visualize the act of rotation on a photon, by measuring the relative phase between the different OAM components of the quantum state. The details of our experiment is presented in the second section of this chapter.

Direct measurement provides a simple technique for characterizing multidimensional states. Yet, the measurement of highdimensional states remains a challenging task. Even for DM the number of measurements that are needed to characterize the state vectors grows linearly with the dimension of the state. Further, a much larger ensemble of identically prepared particles is required for reliable measurement of elements of the state vector in a high-dimensional Hilbert space [126]. The final section of this chapter is allocated to compressive direct measurements (CDM). The CDM method exploits the experimenter's prior knowledge for reducing the number of required measurements. This is achieved by imposing the property of sparsity under the transverse gradient transformation. 


\subsection{Theory and background}

In the first chapter, we overviewed the concept of a weak measurement. A weak measurement is a weak interaction between a quantum system with a pointer state that represents the measurement apparatus. The result of a weak measurement, known as the weak value, was found as

$$
A_{w}=\frac{\left\langle\psi_{f}|\hat{A}| \psi\right\rangle}{\left\langle\psi_{f} \mid \psi\right\rangle} .
$$

Here, $|\psi\rangle$ is the initial state of a system and $\left|\psi_{f}\right\rangle$ is the state of the system after post-selection. It is evident that the weak value can be a complex quantity under general conditions. We showed earlier and that the real and imaginary parts of the weak value are proportional to the expectation values of the position and the momentum of the pointer, respectively.

We now go over the direct measurement formalism, following the treatment by Lundeen and co-workers [10]. We consider a weak measurement of the position projector at point $x$, which can be described via the operator $\hat{A}=|x\rangle\langle x|$, followed by post-selection on an eigenstate of position, $|p\rangle$. The weak value for this measurement can be written as

$$
\left\langle\pi_{x}\right\rangle_{w}=\frac{\langle p \mid x\rangle\langle x \mid \psi\rangle}{\langle p \mid \psi\rangle} .
$$

Substituting the Fourier transform relation between $x$ and $p,\langle p \mid x\rangle=e^{i p x / \hbar}$, we get

$$
\left\langle\pi_{x}\right\rangle_{w}=\frac{e^{i p x / \hbar} \psi(x)}{\phi(p)} .
$$

Here, $\psi(x)$ and $\phi(p)$ are the wave function representations in the position and momentum bases, respectively. This expression can be further simplified by considering the special case of post-selection on $|p=0\rangle$

$$
\left\langle\pi_{x}\right\rangle_{w}=k \psi(x)
$$

It is evident that the weak value is directly proportional to the quantum wave 
function. The proportionality constant, $k=\frac{1}{\phi(0)}$ can be eliminated by imposing the normalization condition of the wave function.

Figure 4.2 shows a schematic of the experimental setup used in [10]. In this realization, the polarization of the photons is used as a two-dimensional pointer, and the wave function is the transverse structure of the optical field. Initially, the beam of photons is filtered to be prepared in the vertical polarization. To perform a weak measurement, the polarization of the optical field is rotated by a small angle, $\alpha$, at the point $x$ by placing a small half wave plate at that location. The strong measurement of momentum is performed by a pinhole located after a Fourier-transforming lens. It is straightforward to show that the weak value is given by $[10]$

$$
\left\langle\pi_{x}\right\rangle_{w}=\frac{1}{\sin \alpha}\left(\left\langle s\left|\sigma_{x}\right| s\right\rangle-i\left\langle s\left|\sigma_{y}\right| s\right\rangle\right) .
$$

Here, $|s\rangle$ denotes the polarization state of the light passing through the pinhole and $\sigma_{x}$ and $\sigma_{y}$ are the x and y Pauli matrices. Classically, the expectation values of $\sigma_{x}$ and $\sigma_{y}$ are equivalent to the $S_{2}$ and $S_{3}$ polarization Stokes parameters.

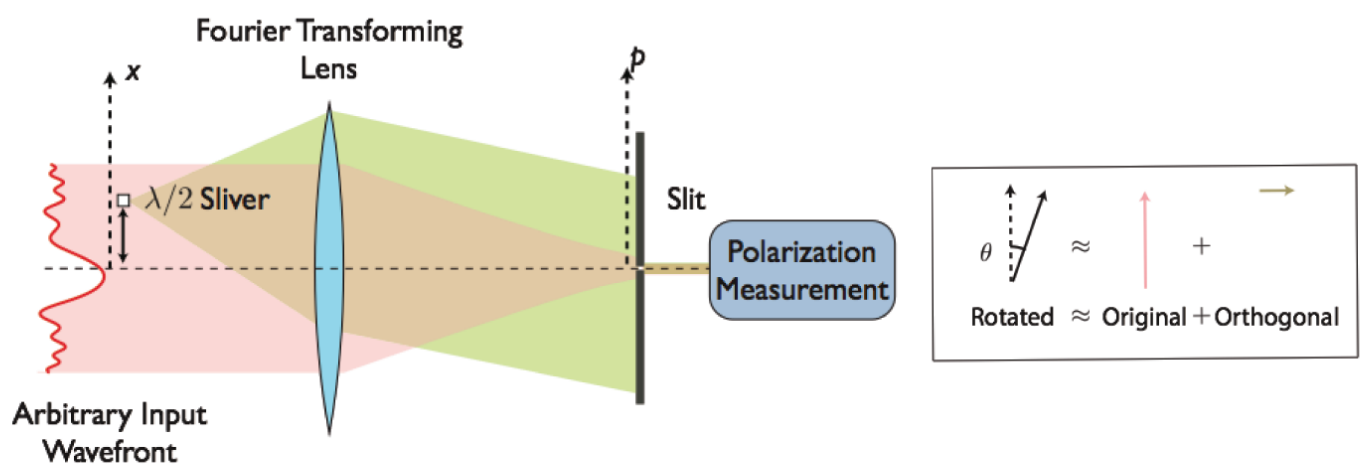

Figure 4.1: Left panel: Experimental setup for direct measurement. Right panel: a small polarization rotation can be pictured as the superposition of the original polarization plus a weak orthogonally polarized component.

Reference[10] proposes and demonstrates the concept of direct measurements. The authors have used direct measurement to characterize the transverse structure of single photons going through a single mode fiber. By scanning the small half wave plate sliver, it is possible to measure the real and imaginary parts 
of the field at each points and then convert the results to amplitude and phase. The authors have repeated the experiment with a classical beam of light and have verified that the results remain identical to those from the experiment with single photons [127]. While a complete comparison of the direct measurement with quantum tomography remains to be drawn, the simplicity of its implementation has provided ample motivation for different experimental realizations $[122,9,8,128]$. 


\subsection{Direct measurement of the OAM state vec- tor}

In this section we use weak measurements of OAM followed by a strong measurement of angular position to directly measure the complex probability amplitudes of a 27-dimensional state in the OAM basis. In this manner, we are able to obtain both the amplitude and the phase of each OAM component within our state?space. We begin by expressing the state of a photon as a superposition of states in the OAM basis as

$$
|\Psi\rangle=\sum_{\ell} a_{\ell}|\ell\rangle
$$

where $a_{\ell}$ are complex probability amplitudes. In direct analogy to a photon's position and linear momentum, the angular position and OAM of a photon form a discrete Fourier conjugate pair [129, 130]. Consequently, any OAM basis state $|\ell\rangle$ is mutually unbiased with respect to any angular position basis state $|\theta\rangle$, i.e. their inner product always has the same magnitude. This property allows us to define a strategic quantity $c=\left\langle\theta_{0}\right\rangle \ell /\left\langle\theta_{0}\right\rangle \Psi$, which is constant with respect to $|\ell\rangle$ for $\theta_{0}=0$. By multiplying our state above by this constant and inserting the identity, we can expand it as

$$
c|\Psi\rangle=c \sum_{\ell}|\ell\rangle\langle\ell\rangle \Psi=\sum_{\ell}|\ell\rangle \frac{\left\langle\theta_{0}\right\rangle \ell\langle\ell\rangle \Psi}{\left\langle\theta_{0}\right\rangle \Psi}=\sum_{\ell}\left\langle\pi_{\ell}\right\rangle_{\mathrm{w}}|\ell\rangle .
$$

Notice here that we have introduced the quantity $\left\langle\pi_{\ell}\right\rangle_{\mathrm{w}}$, which is proportional to the probability amplitude $a_{\ell}$ from Eq. (4.3.1). This is known as the OAM weak value [22], and is equal to the average result obtained by making a weak projection in the OAM basis $\left(\hat{\pi}_{\ell}=|\ell\rangle\langle\ell|\right)$ followed by a strong measurement in the conjugate basis of angular position $(\theta)$. In this manner, the scaled complex probability amplitudes $c a_{\ell}$ can be directly obtained by measuring the OAM weak value $\left\langle\pi_{\ell}\right\rangle_{\mathrm{w}}$ for a finite set of $\ell$. Following this procedure, the constant $c$ can be eliminated by renormalizing the state $|\Psi\rangle$. In order to measure such weak values, 
we utilize a two-system Hamiltonian where the OAM of a photon is coupled to its polarization, which serves as a measurement pointer [10]. We perform a weak projection of OAM by rotating by a small angle the polarization of the OAM mode to be measured. Following this, a strong measurement of angular position is performed via a post-selection of states with $\theta=\theta_{0}$. The OAM weak value is read out by measuring the average change in the photon's linear and circular polarization.

Experimental procedure for measuring the OAM weak value. Performing a weak measurement of OAM at the single photon level is an experimental challenge. In order to do so, we first use our enhanced OAM mode sorter for efficiently separating the OAM components of the incoming photons $[1,4,3]$. This process is depicted in Fig. 4.2 for a single OAM mode. R1 and R2 are custom refractive elements that transform an OAM mode with azimuthal phase variation $e^{i \ell \theta}$ to a momentum mode with position phase variation $e^{i \ell x / a}$. Following a Fourier transform lens (L1), a fan-out hologram implemented on a phase-only spatial light modulator (SLM2) creates three adjacent copies of this momentum mode. Following another Fourier transform lens (not shown in Fig. 4.2), SLM3 is used to remove a relative phase difference introduced in the beam-copying process between the three copies. The resultant momentum mode is three times the size of the original, while also having three times the phase gradient of the original. A second lens (L2) Fourier transforms this larger momentum mode into a position mode at SLM4. This results in well separated OAM modes $(\ell)$ having less than $10 \%$ overlap on average with neighboring modes $(\ell \pm 1)$.

The weak projection of an OAM mode is performed by rotating its polarization by an angle $\alpha=\pi / 9$ (a strong projection would correspond to $\alpha=\pi / 2$ ). We use SLM4 and a quarter-wave plate (QWP0) in double pass to carry out this polarization rotation [131]. QWP1 and HWP1 are used to remove any ellipticity introduced by transmission and reflection through the non-polarizing beamsplitter (NPBS). A strong measurement of angular position is performed by a $10 \mu \mathrm{m}$ slit placed in the Fourier plane of lens L3. Since the plane of the slit is conjugate to the plane where the OAM modes are spatially separated (SLM4), 


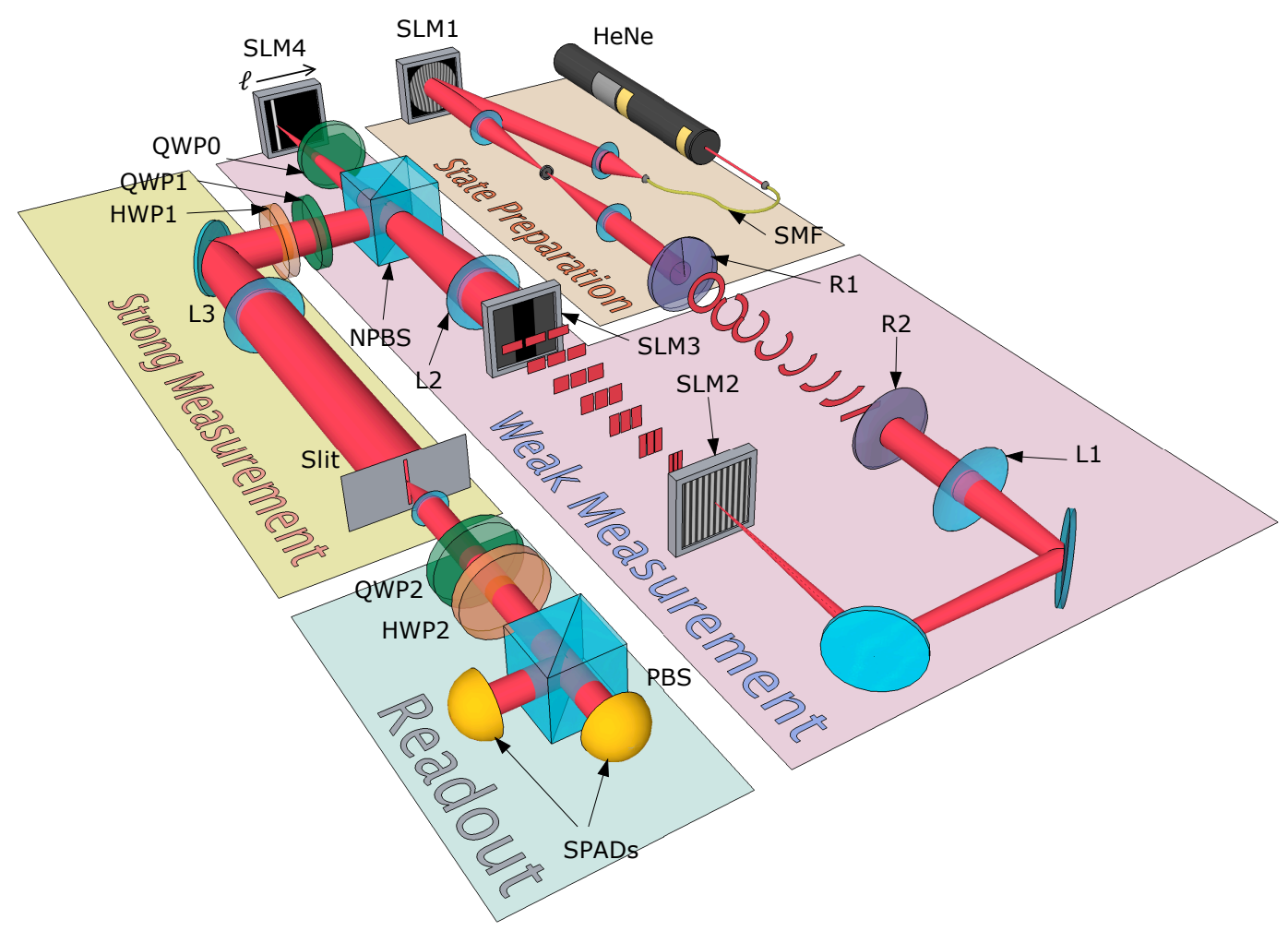

Figure 4.2: Experimental setup for direct measurement of a high-dimensional state vector. State Preparation: A quantum state in an arbitrary superposition of orbital-angularmomentum (OAM) modes is prepared by impressing phase information with a spatial light modulator (SLM1) onto spatially-filtered (SMF) photons from an attenuated HeNe laser. Weak Measurement: A particular OAM mode is weakly projected by rotating its polarization. In order to do so, the OAM modes are first transformed into finite-sized momentum modes by two refractive optical elements made out of PMMA (R1 and R2). Then, a Fourier transform lens (L1) and a fan-out hologram implemented on SLM2 are used to generate three adjacent copies of each momentum mode. The phase between these copies is corrected by SLM3. Another lens (L2) converts these larger momentum modes into well separated position modes at its focus. Finally, a quarter-wave plate (QWP0) used in double-pass with SLM4 are used to rotate the polarization of the OAM mode to be weakly projected. Another quarter-wave and half-wave plate (QWP1 and HWP1) are used to remove any ellipticity introduced by transmission and reflection through the non-polarizing beam splitter (NPBS). Strong measurement: A strong measurement of angular position is performed by Fourier transforming with a lens (L3) and post-selecting state $p=0$ with a $10 \mu \mathrm{m}$ slit. Readout: The OAM weak value $\left\langle\pi_{\ell}\right\rangle_{\mathrm{w}}$ is obtained by measuring the change in the photon polarization in the linear and circular polarization bases. QWP2, HWP2, a polarizing beam splitter (PBS), and two single-photon avalanche detectors (SPADs) are used for this purpose. 
a measurement of linear position by the slit is equivalent to a measurement of angular position.

The average change in the photon's linear and circular polarization is proportional to $\operatorname{Re}\left\langle\pi_{\ell}\right\rangle_{\mathrm{w}}$ and $\operatorname{Im}\left\langle\pi_{\ell}\right\rangle_{\mathrm{w}}$ respectively $[23,10]$. If the initial polarization of the photon is vertical, the OAM weak value is given by

$$
\left\langle\pi_{\ell}\right\rangle_{\mathrm{w}}=\frac{1}{\sin \alpha}\left(\left\langle s_{\mathrm{f}}\left|\hat{\sigma}_{1}\right| s_{\mathrm{f}}\right\rangle-i\left\langle s_{\mathrm{f}}\left|\hat{\sigma}_{2}\right| s_{\mathrm{f}}\right\rangle\right),
$$

where $\alpha$ is the rotation angle, $\hat{\sigma}_{1}$ and $\hat{\sigma}_{2}$ are the first and second Pauli operators, and $\left|s_{\mathrm{f}}\right\rangle$ is the final polarization state of the photon. In order to measure the expectation values of $\hat{\sigma}_{1}$ and $\hat{\sigma}_{2}$, we transform to the linear and circular polarization bases with QWP2 and HWP2, and measure the difference between orthogonal polarization components with a polarizing beamsplitter (PBS) and two singlephoton avalanche detectors (SPADs). In this manner, we directly obtain the scaled complex probability amplitudes $c a_{\ell}$ by scanning the weak measurement through $\ell$ values of \pm 13 . While the size of the OAM state space is unbounded, we are limited to a dimensionality of $d=27$ by our mode transformation technique.

Direct measurement of a 27-dimensional OAM state. The authors of the first work on direct measurement showed this technique to give identical results for heralded single photons and attenuated coherent states [10]. Therefore, in our experiment, photons from a highly attenuated HeNe laser are tailored into a high-dimensional quantum state by impressing a specific OAM distribution on them with SLM1 and a $4 f$ system of lenses (Fig. 4.2) [47]. The laser power is reduced such that probabilistically only one photon is present in our apparatus at any given time. First, we create a sinc-distribution of OAM using a wedgeshaped mask on the SLM. Just as a rectangular aperture diffracts light into a sinc-distribution of linear momenta, photons diffracting through an angular aperture of width $\Delta \theta$ result in a state vector with a sinc-distribution of OAM probability amplitudes [129] 
(a)

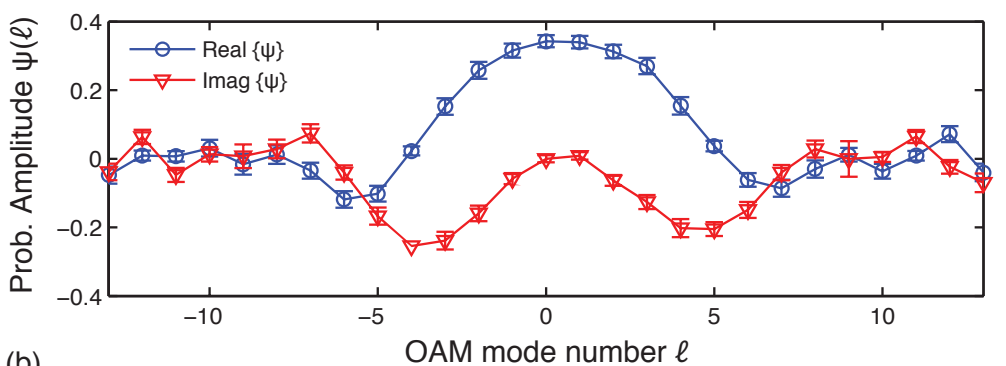

(b)

OAM mode number $\ell$

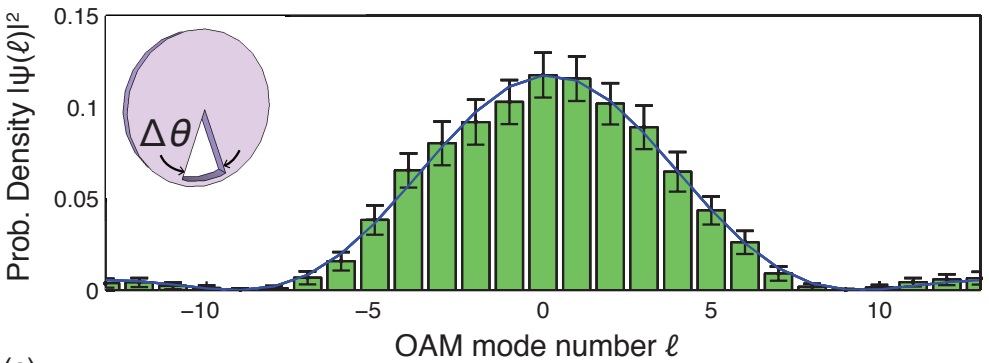

(c)

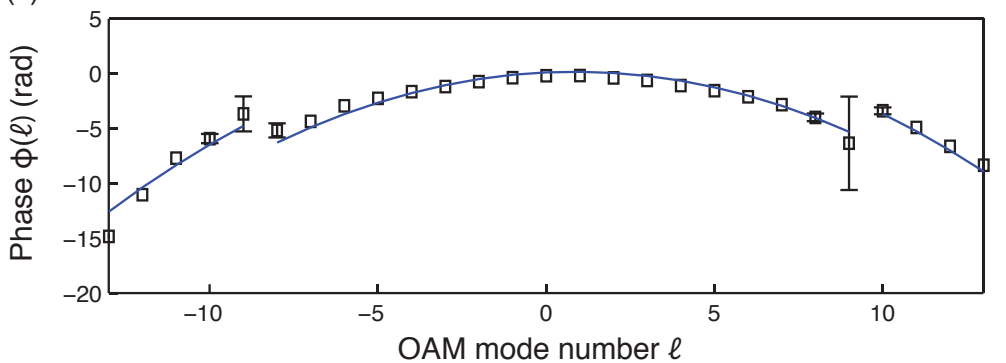

Figure 4.3: Experimental data showing direct measurement of a 27-dimensional state vector in the OAM basis. The state is created by sending photons through an angular aperture of width $\Delta \theta=2 \pi / 9 \mathrm{rad}$ (inset of (b)). (a) The measured real (blue circles) and imaginary parts (red triangles) of the state vector, (b) the calculated probability density $|\Psi(\ell)|^{2}$, and (c) the calculated phase $\phi(\ell)$ are plotted as functions of the OAM quantum number $\ell$ up to a dimensionality of $\ell= \pm 13$. The probability density has a sinc-squared shape. The phase has an asymmetric quadratic shape due to small misalignments in our optical system. Additionally, $\pi$-phase jumps are seen in the phase when the probability amplitude changes sign (not seen in the probability density). Theoretical fits to the probability density and phase are plotted as blue lines. Error bars are calculated by propagating the detector error (due to background light and dark counts) through to all measured quantities. Error bars larger than the symbols are shown. The data shown is the average result obtained from 50 experimental runs.

$$
a_{\ell}=k \operatorname{sinc}\left(\frac{\Delta \theta \ell}{2}\right)
$$


This distribution has a width given by $\Delta \ell=2 \pi / \Delta \theta$, which refers to the mode index of its first null. Using an angular aperture of width $\Delta \theta=2 \pi / 9 \mathrm{rad}$ (inset of Fig. 4.3(b)), we create such an ensemble of identical photons and perform the direct measurement procedure on them. The measured real and imaginary parts of the state vector are plotted in Fig. 4.3(a) as a function of $\ell$. Using these quantities, we calculate the probability density $|\Psi(\ell)|^{2}$ and the phase $\phi(\ell)$, which are plotted in Figs. 4.3(b) and (c). The width of the sinc-squared fit to the probability density is measured to be $\Delta \ell=9.26 \pm 0.21$, which is very close to the value of $\Delta \ell=9$ predicted from theory.

The measured phase plotted in Fig. 4.3(c) has a quadratic form with $\pi$-phase jumps at OAM mode numbers $\ell= \pm 9$. These mode numbers correspond to the probability density minima in Fig. 4.3(b), which is where the sinc-shaped amplitude crosses the $\mathrm{x}$-axis and changes sign. The asymmetric quadratic feature in the phase appears due to small misalignments in our optical system. A $4 f$ imaging system (not shown in Fig. 4.2) is used to magnify the Fourier plane of lens L2 onto SLM4. A misalignment in the $z$-axis of this imaging system appears as a quadratic phase. Further, the asymmetry in the phase is due to a first-order tilt aberration in our optical system, which is simply a result of the plane of SLM2 not being perfectly parallel to the plane of SLM4. Taking these two alignment imperfections into account, we use a quadratic model of the form $a x^{2}+b x+c$ in order to calculate a fit to the phase using a least-squares fitting algorithm in Matlab. Theoretical fits are plotted as blue lines in Fig. 4.3(c). As can be seen, the phase error is unavoidably large when the amplitude approaches zero.

Direct measurement of rotations in the OAM basis. We now use this technique to analyze the effect of rotation on a photon carrying a broad range of angular momenta. Rotation of a state vector by an angle $\theta_{0}$ can be expressed by the unitary operator $\hat{U}=\exp \left(i \hat{L}_{z} \theta_{0}\right)$, where $\hat{L}_{z}$ is the angular momentum operator. Operating on our quantum state $|\Psi\rangle$ with $\hat{U}$, we get 


$$
\left|\Psi^{\prime}\right\rangle=\hat{U}|\Psi\rangle=\sum_{\ell} k \operatorname{sinc}\left(\frac{\Delta \theta \ell}{2}\right) e^{i \ell \theta_{0}}|\ell\rangle
$$

(a)

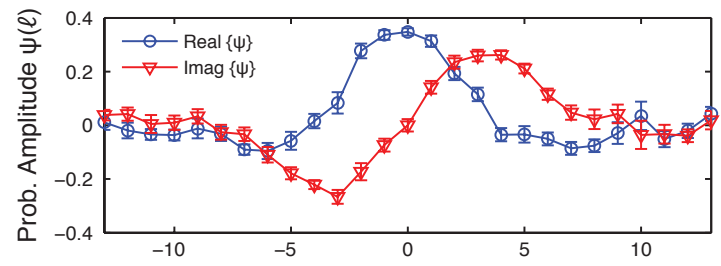

(b)

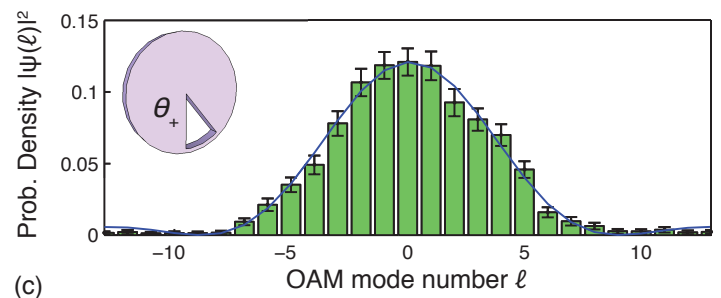

(c)

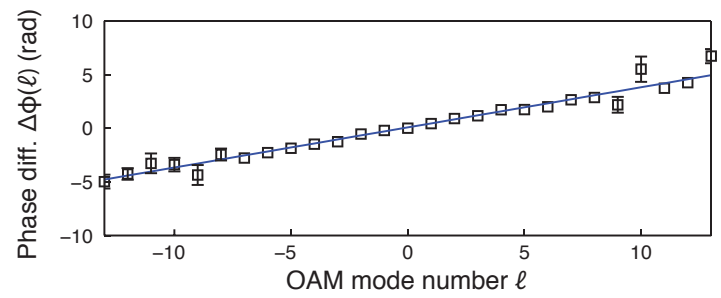

(d)

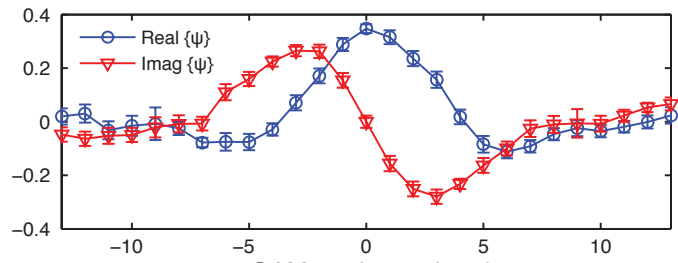

(e)

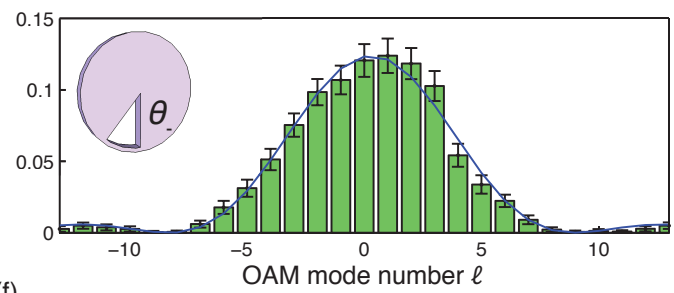

(f)

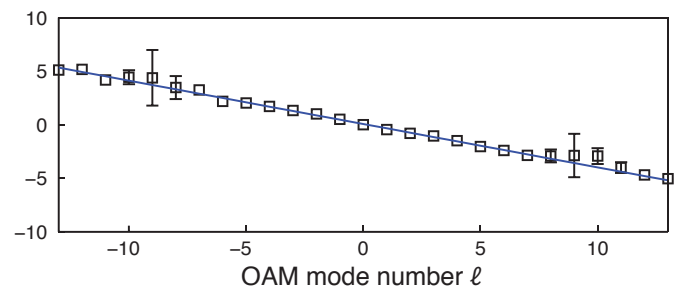

Figure 4.4: Experimental data showing the direct measurement of a rotated high-dimensional state vector. The created state is rotated by angles $\theta_{ \pm}= \pm \pi / 9 \mathrm{rad}$ (insets of (b) and (e)). (a) and (d) The measured real (blue circles) and imaginary parts (red triangles) of the rotated state vectors. (b) and (e) The calculated probability densities $\left|\Psi(\ell)_{ \pm}\right|^{2}$. (c) and (f) The phase difference $\Delta \phi_{ \pm}(\ell)$ between the calculated phase and the phase of the unrotated case from Fig. 4.3(c). Theoretical fits to the probability densities and phases are plotted as blue lines. The linear fits in (c) and (f) are calculated via the process of chi-square minimization, which takes into account the error at each point. Error bars are calculated by propagating the detector error (due to background light and dark counts) through to all measured quantities. Error bars larger than the symbols are shown. The data shown is the average result obtained from 50 experimental runs.

Thus, rotation by an angle $\theta_{0}$ manifests as an $\ell$-dependent phase $e^{i \ell \theta_{0}}$ in the OAM basis. For this reason, the angular momentum operator is called the generator of rotations under the paraxial approximation [132]. To measure this phase, we create a rotated state vector by rotating our angular aperture by an angle $\theta_{+}=\pi / 9 \mathrm{rad}$ (inset of Fig. 4.4(b)). Then, we perform the direct measurement 
procedure as before and measure the real and imaginary parts of the rotated state vector as a function of $\ell$ (Fig. 4.4(a)). The probability density and phase of the state vector are calculated and plotted in Figs. 4.4(b) and (c). For clarity, we subtract the phase of the zero rotation case (Fig. 4.3(c)) from our phase reading, so the effect of rotation is clear. Barring experimental error, the amplitude does not change significantly from the unrotated case (Fig. 4.3(b)). However, the phase of the OAM distribution exhibits a distinct $\ell$-dependent phase ramp with a slope of $0.373 \pm 0.007 \mathrm{rad} /$ mode. This is in close agreement with theory, which predicts the phase to have a form $\phi(\ell)= \pm \pi \ell / 9$, corresponding to a phase ramp with a slope of $\pm 0.35 \mathrm{rad} /$ mode. A linear fit to the phase is calculated by the process of chi-square minimization, which takes into account the phase error at each point. This process is repeated for a negative rotation angle $\theta_{-}=-\pi / 9$ rad, which results in a mostly unchanged probability density, but an $\ell$-dependent phase ramp as expected with a negative slope of $-0.404 \pm 0.007 \mathrm{rad} / \mathrm{mode}$ (Figs. $4.4(\mathrm{~d})-(\mathrm{f}))$.

These results clearly illustrate the relationship between phase and rotation in the OAM basis in that every $\ell$-component acquires a phase proportional to the azimuthal quantum number $\ell$. The measured slopes in both cases are slightly larger than those expected from theory possibly due to errors introduced in the geometrical transformation that is used to spatially separate the OAM modes. The mode sorting process is extremely sensitive to choice of axis, and a very small displacement of the transforming elements R1 and R2 can propagate as a phase error. 


\subsection{Compressive direct measurement}

In this section, we introduce a method which combines the benefits of direct measurement with a novel computational technique known as compressive sensing $[133,134,135,136,137,138]$. Utilizing our approach, the wave function of a high-dimensional state can be estimated with a high fidelity using much fewer number of measurements than the standard direct measurement approach. We begin by establishing the theory of a compressive direct measurement (CDM). We then describe our experimental implementation of CDM, which serves as a direct test for this method. In our experiment, we are able to reconstruct a wave function with only a fraction of the required measurements for a DM measurement with a more than 90 percent fidelity.

As discussed earlier, weak value is the expectation value of a weak measurement followed by a post-selection [22]. Consider a weak measurement of the position operator $\hat{\pi}_{j}=\left|x_{j}\right\rangle\left\langle x_{j}\right|$ at point $x_{j}$ followed by a post-selection on the zero momentum eigenstate $|o\rangle$. The weak value for the above measurement is given by

$$
\pi_{w}=\frac{\left\langle o \mid x_{j}\right\rangle\left\langle x_{j} \mid \psi\right\rangle}{\langle o \mid \psi\rangle}=\frac{\psi\left(x_{j}\right)}{\phi_{0} \sqrt{N}} .
$$

In deriving this formula we have used the Fourier transform property $\phi_{0}=\langle o \mid \psi\rangle$, where $N$ is the dimension of the Hilbert space. We also treat $\phi_{0}$ as a real number as this can always be accounted for by adding a constant phase to the wave function. The above relation indicates that the wave function can be extracted from the weak value, whose real and imaginary parts can be read from the expectation values of two conjugate variable of the pointer (see below) [139]. Note that the transition from the continuous spatial domain to a discrete state vector has been achieved by dividing the continuous coordinate to a finite number of pixels of sufficiently small area.

We now generalize the formalism to a form suitable for compressive sensing. Quantum measurement can be mathematically described by the coupling between the measured system and the measurement pointer. We utilize a two-level 
pointer such as the polarization of photons. Let the initial system-pointer state be

$$
|\Omega\rangle=|\psi\rangle \otimes|V\rangle=\sum_{i=1}^{N} \psi_{i}\left|x_{i}\right\rangle \otimes|V\rangle .
$$

Here, $|\psi\rangle$ is the wave function of interest. We have assumed that the pointer is initially prepared in the vertical eigenstate $|V\rangle$. Consider a weak measurement of the sensing operator $\hat{Q}_{m}=\sum_{j} Q_{m, j} \hat{\pi}_{j}$ which is a linear combination of the position projectors $\hat{\pi}_{j}$ weighted with coefficients $Q_{m, j} \in \mathbb{R}$. The effect of this measurement can be described by making a Taylor series approximation to the measurement's evolution operator

$$
e^{i \alpha \hat{Q}_{m} \otimes \hat{\sigma}_{y}}|\Omega\rangle \simeq|\Omega\rangle+\alpha \sum_{j} Q_{m, j} \psi_{j}\left|x_{j}\right\rangle \otimes|H\rangle
$$

Here, $\hat{\sigma}_{y}=-i|H\rangle\langle V|+i| V\rangle\langle H|$ is a Pauli matrix and $\alpha$ is a constant parameter quantifying the weakness of the measurement. Note that, the polarization at each point $x_{j}$ is rotated around the optical axis by the value $Q_{m, j} \alpha$.

The state of the pointer after post-selection on $|o\rangle$ is given by

$$
\left|s_{m}\right\rangle=|V\rangle+\frac{\alpha}{\phi_{0} \sqrt{N}} \sum_{j} Q_{m, j} \psi_{j}|H\rangle
$$

At this stage the information about the state-vector $\psi_{j}$ is encoded in the polarization of the post-selected photons. The expected values of the polarization of the post-selected state can be obtained as

$$
\begin{aligned}
& \bar{\sigma}_{x, m} \equiv\left\langle s_{m}\left|\hat{\sigma}_{x}\right| s_{m}\right\rangle=\kappa \sum_{j} Q_{m, j} \Re\left[\psi_{j}\right], \\
& \bar{\sigma}_{y, m} \equiv\left\langle s_{m}\left|\hat{\sigma}_{y}\right| s_{m}\right\rangle=-\kappa \sum_{j} Q_{m, j} \Im\left[\psi_{j}\right],
\end{aligned}
$$

where $\hat{\sigma}_{x}=|H\rangle\langle V|+| V\rangle\langle H|$ and $\kappa=\frac{2 \alpha}{\phi_{0} \sqrt{N}}$. In the above relations, $\Re\left[\psi_{j}\right]$ and $\Im\left[\psi_{j}\right]$ are the real and the imaginary parts of $\psi_{j}$ respectively. Combining the 
results $\bar{\sigma}_{x, m}$ and $\bar{\sigma}_{y, m}$ to a complex value $\phi_{m}=\frac{1}{\kappa}\left[\bar{\sigma}_{x, m}-i \bar{\sigma}_{y, m}\right]$ and repeating the measurement several times we obtain $\phi=\mathrm{Q} \psi$. The extended form of this equation reads

$$
\left(\begin{array}{c}
\phi_{1} \\
\phi_{2} \\
\vdots \\
\phi_{M}
\end{array}\right)=\left(\begin{array}{cccc}
Q_{1,1} & Q_{1,2} & \cdots & Q_{1, N} \\
Q_{2,1} & Q_{2,2} & \cdots & Q_{2, N} \\
\vdots & \vdots & \ddots & \vdots \\
Q_{M, 1} & Q_{M, 2} & \cdots & Q_{M, N}
\end{array}\right)\left(\begin{array}{c}
\psi_{1} \\
\psi_{2} \\
\vdots \\
\psi_{N}
\end{array}\right)
$$

Here, $m \in\{1: M\}$ and $n \in\{1: N\}$ where $M$ is the total number of sensing operators and $N$ is the dimension of the Hilbert state of the unknown wave function. To find the wave function $\psi$ we need to solve the above linear system of equations. For the special case $M=N$ the set of equations can be exactly solved for a non-singular matrix $\mathbf{Q}$. However, we are interested in the case where $M \leq N$. The pseudo-inverse of $\mathbf{Q}$ can be used as an optimal linear recovery strategy to find a solution that minimizes the least square error [140, 141]

$$
\boldsymbol{\psi}=\mathrm{Q}^{\dagger}\left(\mathrm{QQ}^{\dagger}\right)^{-1} \phi
$$

Nevertheless, a nonlinear strategy can be used to recover $\boldsymbol{\psi}$ with a far superior quality using the idea of compressive sensing (CS). Consider a linear transformation represented by matrix $\mathbf{T}$. If the wave function under the experiment $\phi$ is known to have very few non-zero coefficients under this transformation, $\boldsymbol{\psi}$ can be recovered by solving the convex optimization problem [140]

$$
\min _{\psi^{\prime}}\left\|\mathbf{T} \psi^{\prime}\right\|_{\ell_{1}}, \text { subject to } \mathrm{Q} \psi^{\prime}=\phi
$$

where $\|\cdot\|_{\ell 1}$ represents the 1-norm. For this approach to work, it is critical that the two bases, defined by $Q$ and $T$, are incoherent [140]. The coherence of the two bases is defined by the square root of the dimension of the bases times the highest fidelity between any pairs of states from the two bases [142]. According to CS theory if the coherence of the two bases is much smaller than unity (dimensionless), by an overwhelming probability, the target wave function 


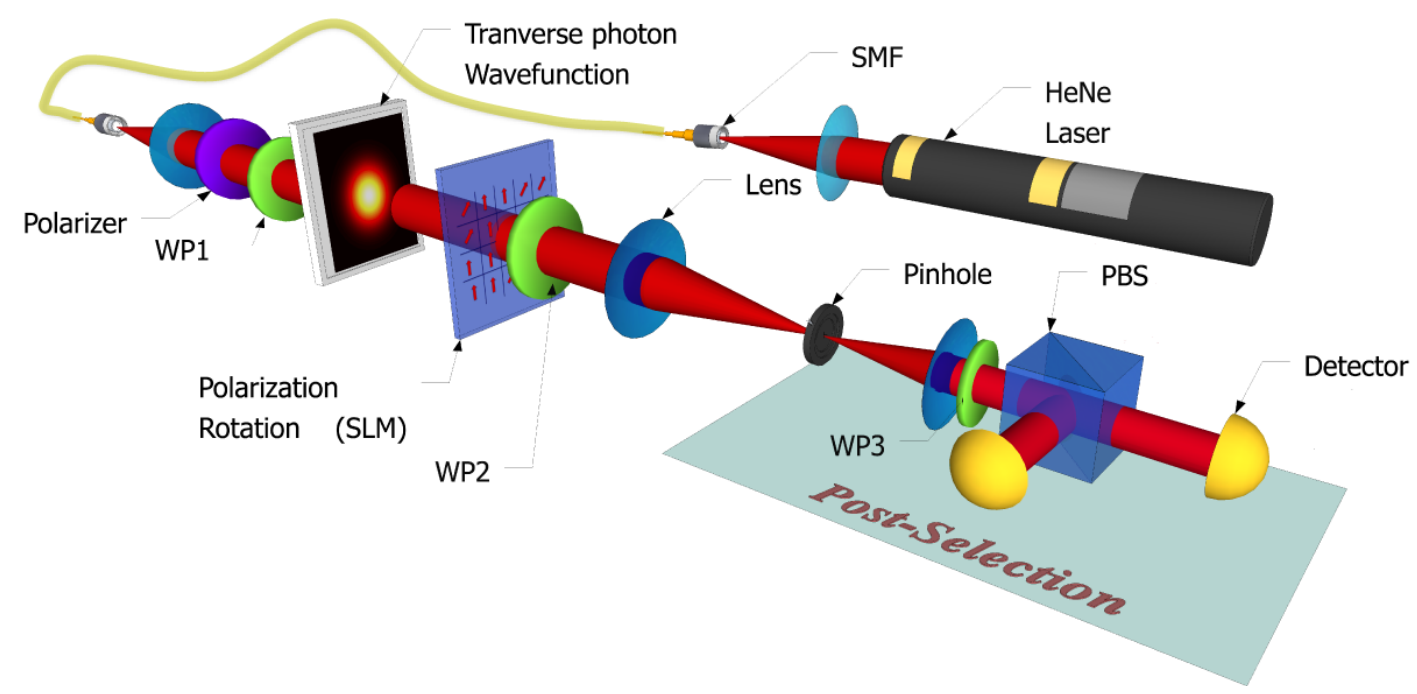

Figure 4.5: A schematic illustration of the experimental setup. Collimated Gaussian beam from the single mode fiber is passed through a polarizer to prepare a vertical polarization. The SLM is used along with two quarter wave plates (WP1 and WP2) to rotate the polarization at each pixel. An $f=50 \mathrm{~cm}$ lens focuses the beam onto a pinhole with a diameter of $10 \mu \mathrm{m}$. The polarization measurement is performed on the light collected from the pinhole using a QWP/HWP (WP3) and a polarizing beam splitter.

$\boldsymbol{\psi}$ can be recovered with $M \geq O[K \log (N)]$ measurements, where $K$ is the number of nonzero components of $\mathbf{T} \boldsymbol{\psi}$ [142]. Functions with spatial correlations are shown to be extremely likely to have sparse coefficients in discrete cosine transform or wavelet transform domains [140, 143]. However, a much simpler variant of Eq. (4.4.9) can be used in practice to achieve results of comparable quality $[140,144]$. In this method the target wave function can be found by minimizing the quantity

$$
\min _{\psi^{\prime}} \sum_{j}\left\|\nabla \psi_{j}^{\prime}\right\|_{\ell_{1}}+\frac{\mu}{2}\left\|\mathrm{Q} \psi^{\prime}-\phi\right\|_{\ell_{2}}^{2}
$$

Here, $\nabla \boldsymbol{\psi}_{j}^{\prime}$ is the discrete gradient of $\boldsymbol{\psi}^{\prime}$ at position $x_{j}$ and $\mu$ is a penalty factor. Heuristically, the minimization of the first term results in a smooth function while the second factor minimizes deviations from the experimental results $\phi$. The optimal value of $\mu$ should be chosen considering the specifics of the target wave function and the signal-to-noise ratio of the experimental data. At the end 


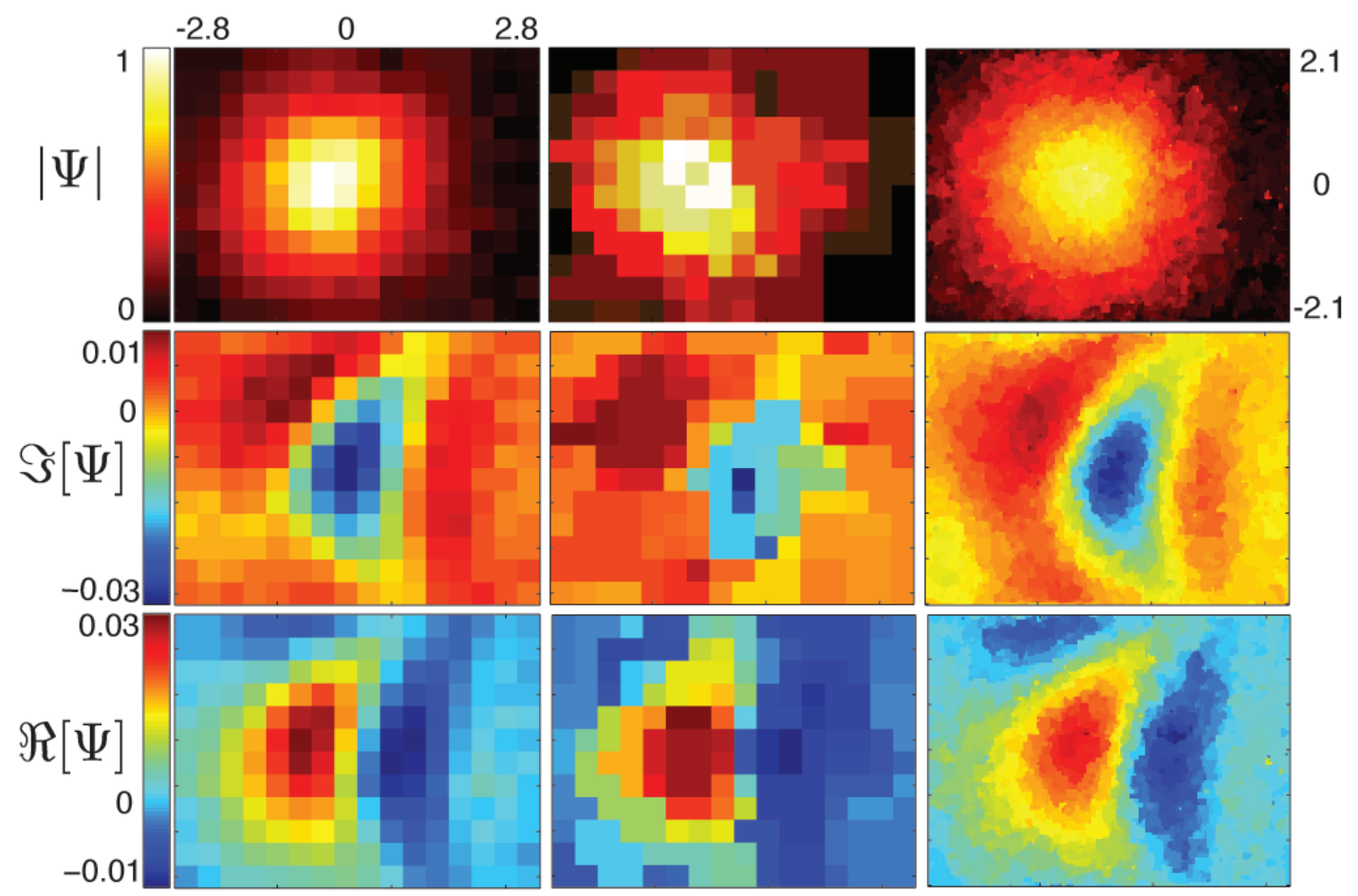

Figure 4.6: The amplitude, real, and imaginary parts of an aberrated Gaussian state from experimental data. The left column presents data from a pixel-by-pixel scan of the state for $\mathrm{N}=192$. The middle column shows the reconstructed wavefront for for $\mathrm{N}=192$, and $M / N=20 \%$ of total measurements from the CDM method. The right column demonstrates reconstruction $\mathrm{N}=19200$, and $M / N=20 \%$ of total measurements. The transverse dimensions of the state are shown in millimeters.

we retrieve the wave function from the solution of the optimization problem as $\left|\psi^{\prime}\right\rangle=\sum_{i=1}^{N} \psi_{i}^{\prime}\left|x_{i}\right\rangle$.

Fig. 4.5 shows the schematics of the experiment. A vertically polarized Gaussian mode is prepared by spatially filtering a He-Ne laser beam with a single mode fiber and passing it through a polarizer. The polarization rotation is performed using a spatial light modulator (SLM) in combination with two quarter wave plates (WP1 and WP2) [145, 146]. The SLM provides the ability to rotate the polarization of the incident beam at every single pixel in a controlled fashion. The post-selection in the momentum basis is done using a Fourier-transforming lens and a single mode pinhole. We retrieve the real part of the weak value using a combination of a half wave plate (WP3) and a polarizing beam splitter (PBS). 
The beams from the output ports of the beam splitter are coupled to single mode fibers that are connected to avalanche photo-diodes (APDs). Similarly, the imaginary part of the weak value is measured by replacing the half wave plate with a quarter wave plate (WP3).

We perform a random polarization rotation of either $20^{\circ}$ or zero at each pixel. The rotated polarization state is given by $\cos \theta|V\rangle+\sin (\theta)|H\rangle$ where $\sin \theta=Q_{m, j} \alpha$. This corresponds to $Q_{m, j}$ values of 1 and 0 and $\alpha=0.349$. For different values of $m$, we load different pre-generated sensing vectors $\mathbf{Q}_{m}$ onto the SLM and repeat the experiment. The wave function is then retrieved via post processing on a computer. We use the algorithm known as Total Variation Minimization by Augmented Lagrangian and Alternating Direction (TVAL3) [147] to solve Eq. (4.4.10). In our experiment, we have used values of $\mu$ ranged from $2^{4}$ to $2^{13}$ (a larger value of $\mu$ results in a closer agreement between the reconstructed state and the experimental data). Our target wave function is a collimated Gaussian beam from a single-mode fiber. The lens after the fiber is slightly displaced to create an aberrated wavefront. This create a complex wave function made from both real and imaginary parts. We reconstruct the wave function from the conventional direct measurement method using Eq. (4.4.1). The real and imaginary parts from a pixel-by-pixel raster scan are shown on the left column of Fig. 4.6 for a $N=12 \times 16=192$ dimensional Hilbert space. The real and imaginary parts of the wave function reconstructed from CDM using $N=192$ and $M / N=20 \%$ are shown on the middle column. It can be seen that the main features of the state are retrieved with as few as $20 \%$ of the total number of measurements used in the left column. It should be emphasized that the minimum number of required measurement for an accurate reconstruction is proportional to the sparsity of the signal. Our algorithm uses sparsity with respect to the gradient transformation, according to Eq. (4.4.10). In order to achieve a more sparse signal, we have done a fine grain measurement of the same state at the resolution of $N=120 \times 160=19200$. The wave function reconstructed from CDM using $M / N=20 \%$ is shown on the right column of Fig. 4.6. Due to increased sparsity of the state in the larger Hilbert space, a very detailed reconstruction can be achieved with $20 \%$ of the total number of 


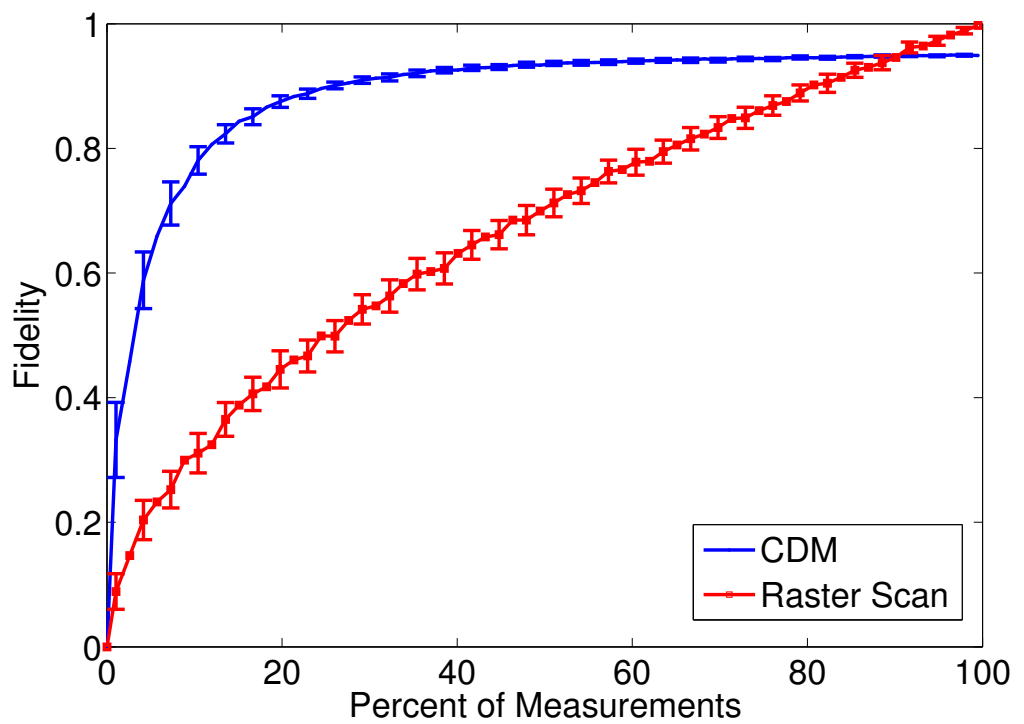

Figure 4.7: The fidelity of the reconstructed state with the target wave function as a function of the percentage of the total measurements. The fidelity of the state reconstructed with CDM is shown in blue. The fidelity of the state reconstructed from a partial pixel-by-pixel scan with the same number of measurements is shown in red for comparison. The error-bars represent standard deviation calculated from 100 repetitions of the experiment (error-bars are shown at every second data point for visual clarity).

measurements.

To provide a quantitive comparison of the two methods we calculate the fidelity between the retrieved state $\left|\psi^{\prime}\right\rangle$ and the target state $|\psi\rangle$ from a full pixel-by-pixel scan as

$$
F\left(\left|\psi^{\prime}\right\rangle,|\psi\rangle\right)=\left|\left\langle\psi^{\prime} \mid \psi\right\rangle\right|
$$

The results are shown in Fig. 4.7. The horizontal axis corresponds to the percentage of the measurements $\left(100 \times \frac{M}{N}\right)$. The blue curve shows the fidelity of the state reconstructed with the CDM method. The red curve represents the average fidelity of state reconstructed with Eq. (4.4.8) using the data from a partial pixel-by-pixel measurement of $M$ randomly chosen points. It is seen from the figure that the compressive method results in a drastic increase of fidelity for the first few measurement and gradually settles to a value close to 1 . As an example of the usefulness of the compressive method, a fidelity as high as $90 \%$ is achieved by performing only $25 \%$ of measurements, while the conventional direct 
measurement needs approximately $80 \%$ of all the measurements to achieve the same fidelity.

To further demonstrate the accuracy of our method we have used it to measure a custom state prepared using a phase mask depicting letters $\mathrm{U}$ and $\mathrm{R}$ with a phase jump of $\pi / 2$. The phase mask is prepared via an additional spatial light modulator illuminated with the Gaussian beam from the laser and the state is imaged onto the second SLM which is used for polarization rotation. Figure 4.8 shows the amplitude and the phase of the reconstructed state with $M / N=20 \%$ of the total measurements. Notice that while the amplitude is relatively uniform, the phase shows the letters $U$ and $R$ with a remarkable accuracy. It should be emphasized that the measurement of a state of such high dimensions is extremely time consuming via a pixel-by-pixel scanning. In our approach, we perform a weak measurement on approximately half of all the pixels at each time. Due to this, the change in the state of the pointer (i.e. the polarization of the beam after the pinhole) is much more pronounced as compared to the conventional DM where only one pixel would be weakly measured. The speed-up factor can be estimated considering that the strength of the signal measured in the laboratory is proportional to the value of the second term in Eq. 4.4.4. It is easy to check that the magnitude of this term is on average $\sqrt{N} / 2$ times larger in the case when half of $Q_{m, j}$ are set to one. For the case of our experiment with $N=19200$, and $M / N=20 \%$, our approach provides a $\sim 350$-fold speed-up in the measurement procedure.

It should be emphasized that our specific experimental realization of the CDM method can be described using classical physics. The measured wave function in this case is the spatial mode of photons which is equivalent to the electric field of paraxial light beams in the classical limit [148]. Since the experiment is designed to measure the spatial mode, it is insensitive to the number of excitations of the field (i.e. the number of photons). Subsequently, the results of the experiment would be the same for a source of single photons, heralded single photons or a strong laser beam provided that they are prepared in the same spatial and polarization modes. However, the language of quantum mechanics provides a simpler description, with a broader range of applicability that 

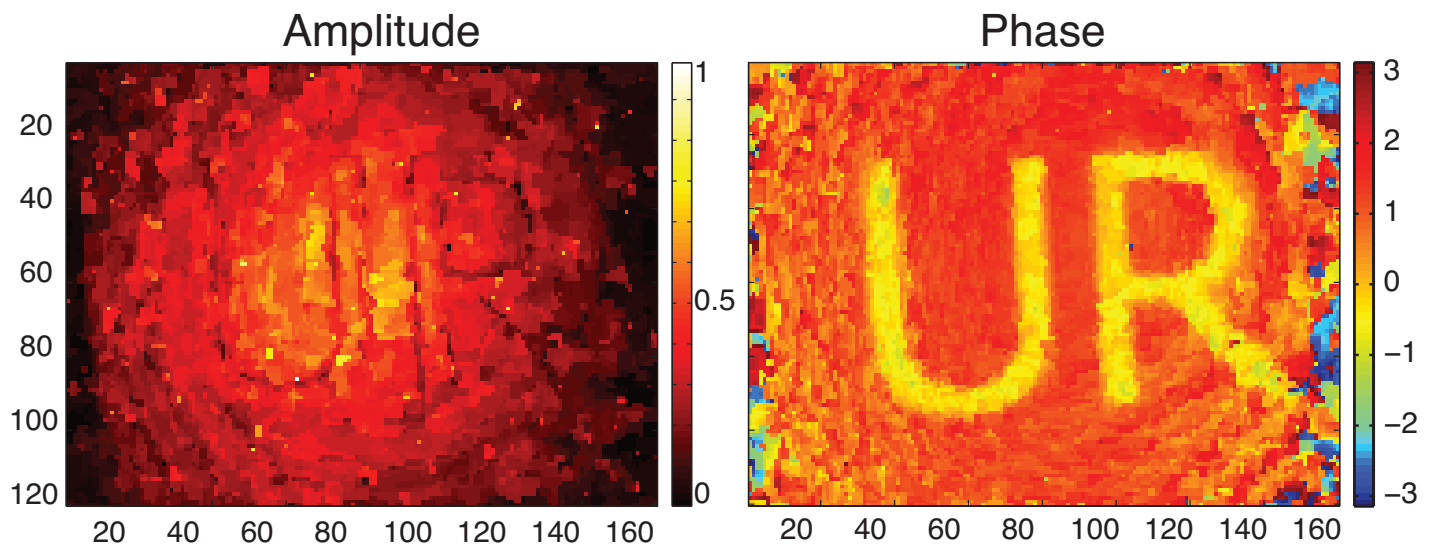

Figure 4.8: The amplitude and phase of a Gaussian mode illuminating a custom phase mask (the initials of the University of Rochester). The data is reconstructed by the CDM method with $\mathrm{N}=19200$, and $M / N=20 \%$ of total measurements.

includes fundamentally quantum mechanical states such as electron beams.

Recently, compressive sensing has been used to simultaneously measure the position and momentum distributions of photons with the aid of partially projective measurements [149]. This method requires parallel simultaneous measurements performed by a camera for characterizing probability distributions of conjugate variables. In contrast, the exclusive use of single-pixel detectors in our technique provides an alternative to inefficient and costly arrayed detectors for quantum optics [150] and terahertz applications [151]. Additionally, the use of a single-pixel detector is advantageous to parallel multi-pixel measurements in terms of signal-to-noise ratio. Last but not least, we emphasize that the measurement of position and momentum probability distributions is not identical to the full measurement of the wave function. The measurement results from the method in Ref. [149] need to be processed using recursive numerical algorithms to retrieve the phase information, which is crucial for determination of the wave function. Such algorithms are proved to provide a unique solution only for restrictive sets of conditions [152]. 


\subsection{Summary}

Determining an unknown wave function is of fundamental importance in quantum mechanics. Despite many seminal contributions, in practice this task remains challenging, especially for high-dimensional states. In the first section of this chapter, we have overviewed the direct measurement approach, introduced by Lundeen et. al. This method provides a ground for meeting the high-dimensionality challenge [10]. We have presented our implementation of direct measurements for the azimuthal degree of freedom in the second section of this chapter. By performing a series of weak and strong measurements of orbital angular momentum and the azimuthal angle we have been able to retrieve the complex OAM state in a 27-dimensional Hilbert space. We have used this method to characterize a superposition of the OAM modes. Further, our method provides the relative phase between the OAM components of the states, making it possible to directly sense angular rotations. In the third section of this chapter, we have introduced the idea of compressive direct measurement (CDM). In this method, we combine the computational efficiency of compressive sensing with the experimental simplicity of the direct measurement in determining the wave function of an a priori unknown state. Our experimental results demonstrate that a compressive variation of the direct measurement allows an accurate determination of a 192-dimensional state with a fidelity of $90 \%$ using only 25 percent of measurements that are needed for the conventional direct measurement approach. This method provides an easy means of characterizing high-dimensional systems in the labs. In addition, the technique can be used for applications which involve a classical beam of light such as wavefront sensing of Terahertz waves. 


\section{Chapter 5}

\section{Characterization of mixed OAM states}

\subsection{Introduction}

In the previous chapters, we discussed the concept of photonic orbital angular momentum and its potential applications in quantum information. We also overviewed methods of efficient generation, projective measurement, and direct measurement of the wave function for the orbital angular momentum of light. The focus of this chapter will be the characterization of a mixed quantum state in the basis of OAM.

Quantum mechanically, a discrete pure state can be described with a complex state vector. A projective measurement provides the OAM spectrum of an optical state, which is proportional to the modulus of the wave vector. A variety of experimental methods can achieve projective measurement of OAM by using phase plates [32], spatial light modulators (SLM) [39], q-plates [107] and custom designed OAM mode sorters, like the one previously described in this thesis $[40,3,1]$. However, determination of the OAM state vector also requires measurement of the relative phase among different basis vectors. This task was recently achieved by using weak measurements, as described in an earlier chapter of this thesis [9]. 
The state vector adequately describes a pure quantum system. However, pure states are only a restricted set of physical states in the Hilbert space, because the vast majority of conceivable states are mixed states [153]. The most general description of a quantum state requires knowledge of its density matrix, or an equivalent phase-space quasi-probability distribution, such as the Wigner distribution [154]. Moreover, the post-selection loss from weak-valuebased techniques has been shown to result in a suboptimal estimation strategy [155]. This issue has been a topic of heated debate lately [156, 157, 158].

In principle, it is possible to determine the density matrix with quantum state tomography (QST) $[118,117]$. However, QST in the OAM basis requires the capability to perform projective measurements on arbitrary superpositions of two or more OAM eigenstates, a task that remains challenging due to technical limitations. For the restricted case of two OAM eigenstates, this task can be realized with a q-plate $[20,107]$. Projection on an arbitrary superposition state can be achieved with an SLM. Nonetheless, the variations in the efficiency of measuring different OAM modes and the cross-talk between neighboring modes critically limits the accuracy of this approach [159].

In this chapter, we propose and demonstrate a method for obtaining the quantum density matrix in the basis of OAM. We achieve this goal by directly finding the density matrix elements in the basis of azimuthal angle and then mapping it onto the OAM basis. As we show, one can make use of the periodicity of the state in the basis of azimuthal angle to establish a simple approach for directly measuring the elements of density matrix. Our scheme is entirely based on strong measurements and is realized with a photon efficiency of unity. We provide results for the experimental characterization of pure and mixed OAM states imposed on the transverse structure of classical and quantum sources of light 


\subsection{Measurement of the density matrix}

We begin our analysis by considering a quantum system with an unknown density matrix in the basis of azimuthal angle, $\rho$. Next, we introduce an ancillary qubit state used as a pointer, that is prepared in the known initial state $|+\rangle=(|H\rangle+|V\rangle) / \sqrt{2}$. For the optical implementation of our technique we utilize the polarization of photons as the pointer. For this reason, we refer to two qubit eigenstates as the vertical and horizontal polarization states, $|V\rangle$ and $|H\rangle$. The joint state of our unknown quantum system and the pointer is given by

$$
\hat{\Omega}=\hat{\rho} \otimes|+\rangle\langle+| .
$$

In the next step, we consider the evolution of the joint system-pointer state under the unitary evolution characterized by the operator $\hat{U}=\exp \left(-i \tau \hat{L} \otimes \hat{\sigma}_{z}\right)$. Here, $\hat{L}$ is the orbital angular momentum operator of the quantum state and $\hat{\sigma}_{z}=|H\rangle\langle H|-| V\rangle\langle V|$, which is one of the Pauli operators for the pointer. Heuristically, the operator $\hat{U}$ describes a polarization-sensitive rotation by the angle $\tau$. After this transformation, the system-pointer state can be written as

$$
\hat{\Lambda}=\hat{U}^{\dagger} \hat{\Omega} \hat{U} \text {. }
$$

It is straightforward to verify that the unitary interaction $\hat{U}$ results in an entangled system-pointer state. Post-selection on a specific angular state $\theta$ leads to a reduced density matrix in the Hilbert space of the pointer:

$$
\hat{\sigma}=\frac{\langle\theta|\hat{\Lambda}| \theta\rangle}{\operatorname{Tr}[\langle\theta|\hat{\Lambda}| \theta\rangle]} .
$$

We can directly find the unknown density matrix of the quantum system, $\hat{\rho}$, by measuring the expectation values of the Pauli operators for the pointer. This calculation can be performed by using the well-known property of the angular eigenstates, $\exp (-i \tau \hat{L})|\theta\rangle=|\theta+\tau\rangle$. Here, we have $\theta_{-}=[\theta-\tau]$ and $\theta_{+}=[\theta+\tau]$. 
Using this notation we find that

$$
\begin{aligned}
& \left\langle\hat{\sigma}_{x}(\theta, \tau)\right\rangle=\operatorname{Tr}\left[\hat{\sigma}_{x} \hat{\sigma}\right]=\frac{2}{N(\theta, \tau)} \operatorname{Re}\left[\left\langle\theta_{+}|\hat{\rho}| \theta_{-}\right\rangle\right], \\
& \left\langle\hat{\sigma}_{y}(\theta, \tau)\right\rangle=\operatorname{Tr}\left[\hat{\sigma}_{y} \hat{\sigma}\right]=\frac{2}{N(\theta, \tau)} \operatorname{Im}\left[\left\langle\theta_{+}|\hat{\rho}| \theta_{-}\right\rangle\right] .
\end{aligned}
$$

Here, we have $N(\theta, \tau)=\operatorname{Tr}[\langle\theta|\hat{\Lambda}| \theta\rangle]=\left\langle\theta_{-}|\hat{\rho}| \theta_{-}\right\rangle+\left\langle\theta_{+}|\hat{\rho}| \theta_{+}\right\rangle$.

We can simplify this result by working in a $(2 N+1)$-dimensional state space spanned by the $\hat{L}$ eigenvectors $|\ell\rangle$ with $\{|\ell| \leq N\}$. In this subspace, an angular eigenstate can be constructed with the discrete Fourier transformation

$$
\left|\theta_{0}\right\rangle=\frac{1}{\sqrt{(2 N+1)}} \sum_{\ell=-N}^{\ell=+N} e^{-i \theta_{0} \ell}|\ell\rangle .
$$

Here, $\left|\theta_{0}\right\rangle$ is the zero-angle state. Subsequently, any other eigenstate of angle can be found using the shift property under rotation

$$
\exp (-i \theta \hat{L})\left|\theta_{0}\right\rangle=\left|\theta+\theta_{0}\right\rangle
$$

The angular states defined above have previously been introduced in the literature for development of a Hermitian operator for rotation angle [132] and also for generalization of the BB-84 QKD protocol for OAM basis [5, 84].

We simplify Eq. (5.2.4) by taking the rotation angles and the post-selection angles to possess discrete values. Doing so, we find that the elements of density matrix in a discrete basis of angular states take the following form

$$
\begin{aligned}
\left\langle\theta_{m}|\hat{\rho}| \theta_{n}\right\rangle= & \frac{N\left(\alpha_{m n}, \beta_{m n}\right)}{2} \times \\
& {\left[\left\langle\hat{\sigma}_{x}\left(\alpha_{m n}, \beta_{m n}\right)\right\rangle+i\left\langle\hat{\sigma}_{y}\left(\alpha_{m n}, \beta_{m n}\right)\right\rangle\right] . }
\end{aligned}
$$

Here, we have $\theta_{m}=2 \pi m /(2 N+1), \alpha_{m n}=\left(\theta_{m}+\theta_{n}\right) / 2$, and $\beta_{m n}=\left(\theta_{m}-\theta_{n}\right) / 2$. Hence, the expression above provides a procedure for measuring each element of the density matrix $\rho_{m n}=\left\langle\theta_{m}|\hat{\rho}| \theta_{n}\right\rangle$ by performing a rotation of $\beta_{m n}$, followed by a post-selection on an an angle state at the angular position $\alpha_{m n}$. Our approach 
separates the real and imaginary parts of the density matrix, and each part can be found directly by characterizing the two conjugate variables $\hat{\sigma}_{x}$ and $\hat{\sigma}_{y}$ of the pointer. Note that the entire procedure is based on strong measurements and, as we outline in the experimental section, we can realize in parallel the post-selection on the angle states $\left|\alpha_{m n}\right\rangle$ with an array of detectors and therefore avoid the post-selection loss.

The approach detailed above provides the density matrix in the $(2 N+1)$-dimensional basis of $\left|\theta_{n}\right\rangle$. Once we find the density matrix in the angular basis, we can use the relation $\left\langle\theta_{n} \mid \ell\right\rangle=\exp \left(-i \theta_{n} \ell\right) /(2 N+1)$ to find the elements of the density matrix in the basis of OAM as

$$
\rho_{\ell, \ell^{\prime}}=\sum_{\{m, n\}=-N}^{N}\left\langle\ell^{\prime} \mid \theta_{m}\right\rangle\left\langle\theta_{m}|\hat{\rho}| \theta_{n}\right\rangle\left\langle\theta_{n} \mid \ell\right\rangle .
$$

\subsection{Experimental realization}

The left panel of Fig. 5.1 illustrates our experimental setup. We use the light beam from a $3 \mathrm{~mW}$ He-Ne laser $(633 \mathrm{~nm})$, that is coupled to a single-mode fiber $(\mathrm{SMF})$ and then expanded to a spot size of $1.8 \mathrm{~cm}$. The laser beam homogeneously illuminates the display of the SLM, which has an active area of $(9.3 \times 7$ $\mathrm{mm}^{2}$ ). The SLM is used to realize computer generated holograms for creating arbitrary spatial structures [47]. We use a Dove prism inside a Sagnac interferometer for realizing the transformation in Eq. (5.2.2). The beam is set to the $45^{\circ}$ polarization state before the interferometer. We use a quarter- and a half-wave plate along with a polarizing beam splitter (PBS) for realizing the measurement of $\left\langle\hat{\sigma}_{x}\right\rangle$ and $\left\langle\hat{\sigma}_{y}\right\rangle$.

It is possible to experimentally realize a projection onto an angular states defined in Eq. (5.2.5) with a series of custom optical elements [1, 2]. However, post-selection on an angular wedge with sharp boundaries is a much simpler task that provides all necessary information for finding the density matrix in the OAM basis. We achieve this task by recording the intensity of the beam at the two output ports of the PBS with a charge-coupled device (CCD) camera. 

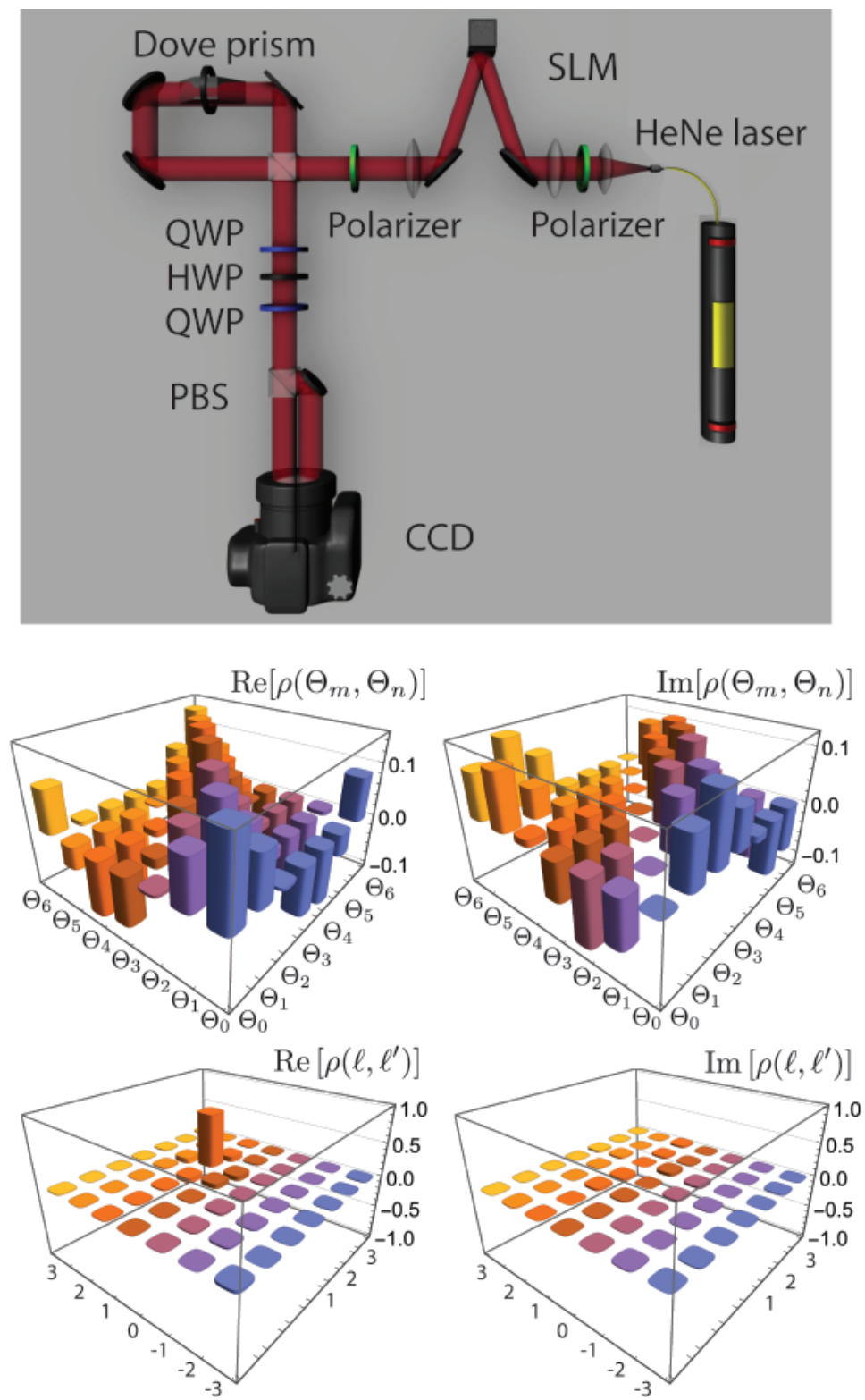

Figure 5.1: Characterization of the transverse structure of classical light. Top panel: The light beam from a HeNe laser illuminates a phase-only spatial light modulator. The polarization state of the beam is prepared by a polarizer. A Dove prism located inside a Sagnac interferometer causes a rotation in opposite directions of each of the counter-propagating beams. Two quarter wave plates along with a half wave is used along with a polarizing beam splitter for characterizing the polarization of the output beam. Bottom panel: Experimental results for characterization of an OAM mode with $\ell=1$. The top plot shows the density matrix in the (discretized) basis of azimuthal angle and the plot in the bottom is the measured density matrix in the OAM basis. 
Once we record the intensity in form of an image, it can be binned to a sequence of numbers that correspond to post-selection on multiple angular states. To approximate the $(2 N+1)$ angular states, we define the angular wedges as

$$
\left|\Theta_{n}\right\rangle=\frac{\sqrt{2 \pi}}{2 N+1} \sum_{\ell=-\infty}^{\infty} \operatorname{sinc}\left(\frac{\ell \pi}{2 N+1}\right) e^{-i \alpha_{n} \ell}|\ell\rangle .
$$

Here, we have $n=1,2, \ldots, 2 N+1$, and $\operatorname{sinc}(x)=\sin (x) / x$. It is easy to check that the angular wedge modes defined above possess the same mean angular positions as the angular states previously defined in Eq. (5.2.5).

The shift property of the angular modes Eq. (5.2.6) is the crucial property in our theoretical analysis above. It is straightforward to show that the angular wedge states $\left|W_{n}\right\rangle$ satisfy this property. Hence, we can directly find the density matrix elements $\left\langle W_{m}|\hat{\rho}| W_{n}\right\rangle$. We subsequently convert our measurement results to the OAM basis using Eq. (5.2.8) by substituting $\left\langle\theta_{n} \mid \ell\right\rangle$ with $\left\langle W_{n} \mid \ell\right\rangle$. Note that the states $\left|W_{n}\right\rangle$ reside in a larger Hilbert states than the one spanned by $|\ell\rangle$ with $|\ell| \leq N$. Nevertheless, a measurement in the basis $\left|W_{n}\right\rangle$ provides sufficient information to provide the density matrix, provided the prior information that the state under characterization resides in the subspace spanned by $|\ell\rangle$ with $|\ell| \leq N$.

To confirm our characterization method, we test it on a series of different states. The bottom panel of Fig. 5.1 shows experimental results for the characterization of an $|\ell=1\rangle$ OAM mode generated by the SLM. The top plot shows the density matrix in the angular wedge basis, whereas the bottom plot demonstrates the density matrix transformed into the OAM basis. We calculate the fidelity of the characterized state with $|\ell=1\rangle$ as $90 \%$, testifying to the high quality of the generation and the characterization procedure. We have used the standard method of maximum-likelihood estimation to find positive-definite density matrices from the experimental data [160].

As another test, we generate and characterize an equal superposition of the OAM states $|\ell=1\rangle$ and $|\ell=-1\rangle$. A pure superposition state is generated directly through the use of a computer generated hologram. To create a mixed 

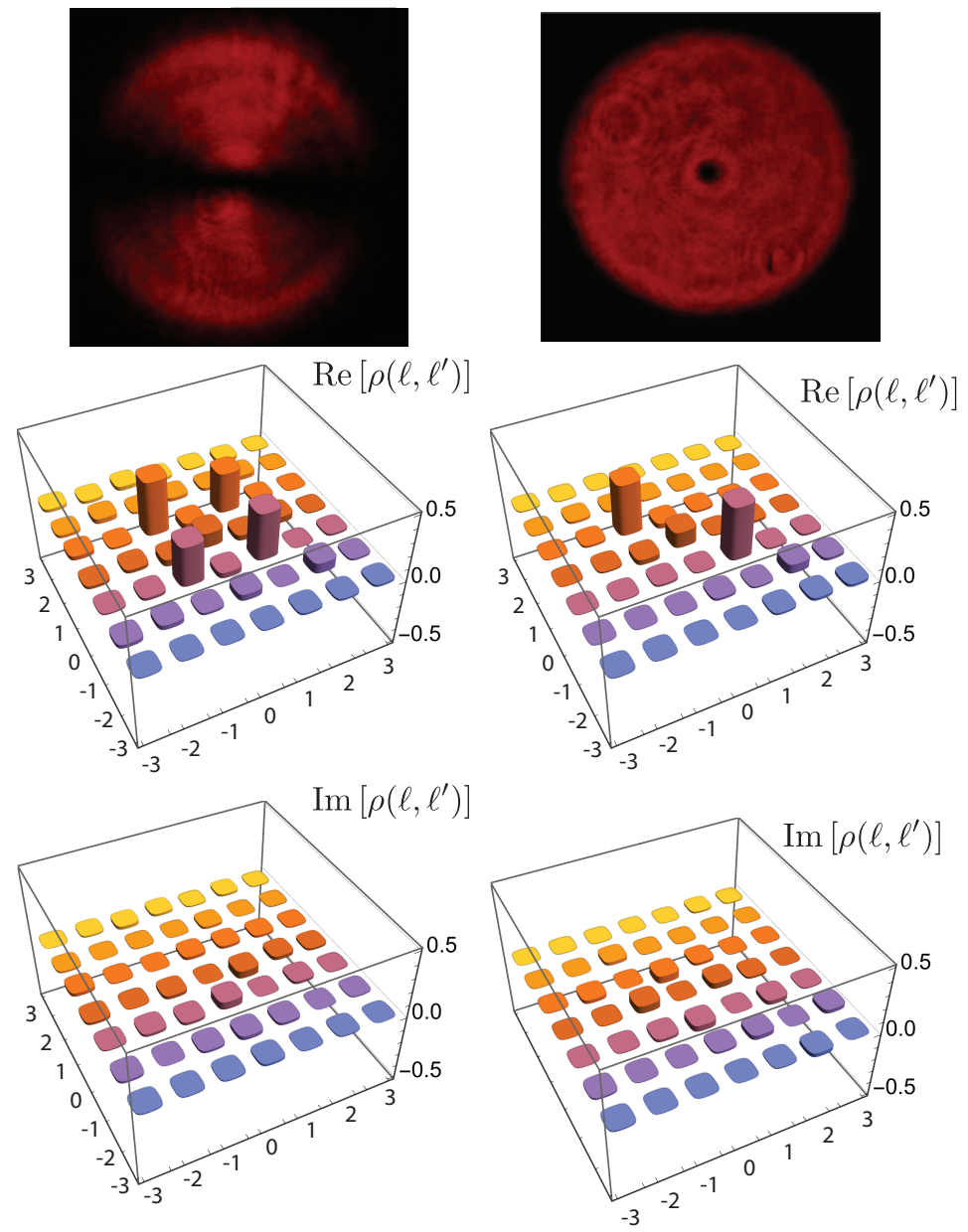

Figure 5.2: Characterization of pure and mixed superposition states. Top panel: The intensity pattern of a pure (left) and mixed (right) superposition of $\ell=1$ and $\ell=-1$ OAM modes with equal weights. Bottom panel: The real and imaginary parts of the OAM density matrix from experiment.

state, we use a computer program to randomly switch the SLM between two holograms designed for generating $\ell=1$ and $\ell=-1$ modes. The mode switching occurs at a rate of $60 \mathrm{~Hz}$, and we use a long (10 s) exposure time on the CCD to guarantee uniform averaging over the changing beam structure. Figure 5.2 shows the intensity patterns and the measured density matrix for the two states. The degree of coherence between the OAM states $|\ell=1\rangle$ and $|\ell=-1\rangle$ 
can be calculated as

$$
\gamma=\frac{\left|\rho_{\{-1,1\}}\right|}{\sqrt{\left|\rho_{\{1,1\}}\right|\left|\rho_{\{-1,-1\}}\right|}} .
$$

We calculate the degree of coherence for the two states as $\gamma_{\text {pure }}=0.80$ and $\gamma_{\text {mixed }}=0.06$. For the pure superposition state, we attribute the slight reduction from unity of the degree of coherence to the imperfections in the generation of the state and the averaging over the non-uniform radial structure of the laser beam. In addition to the results presented above, we have tested our method on a number of different states in the angular and OAM bases (see Appendix B for more information).

The high photon efficiency of our method makes it suitable for characterization of quantum sources of light, which are often limited in the photon flux. We test our method by characterizing the transverse structure of heralded single photons using the setup depicted in Fig. 5.3. We generate pairs of photons by pumping a poled potassium titanyl phosphate crystal (PPKTP) with the beam from a $405 \mathrm{~nm}$ laser diode [161]. The parametric down-conversion process converts a photon of the pump beam to a pair of signal and an idler photons at the wavelength of $790 \mathrm{~nm}$ and $830 \mathrm{~nm}$ respectively. We separate the two photons of each pair by using a dichroic mirror. The idler photons are then collected with a lens and detected using an an avalanche photo-diode (APD). The signal photons are sent through a q-plate that is sandwiched between two crossed polarizers. We use a q-plate with a charge of $1 / 2$ to shape the transverse structure of the photon to a superposition of $|\ell=1\rangle$ and $|\ell=-1\rangle$ states [20]. The structured photons are sent through the Sagnac interferometer described above. We use an Andor iStar intensified charge coupled device (ICCD) camera for detecting the heralded single photons [162]. Each detection event is triggered by the electronic signal from the APD in a 5 ns time window. Figure 5.3 displays the structure of the shaped signal beam from a 1200 sec exposure. We combine our measurement results for the different rotation angles of the dove prism to find the density matrix (see the bottom panel of Fig. 5.3). It can be verified that the density matrix closely resembles the one from measurement of a $|\ell=1\rangle$ and $|\ell=-1\rangle$ previously 

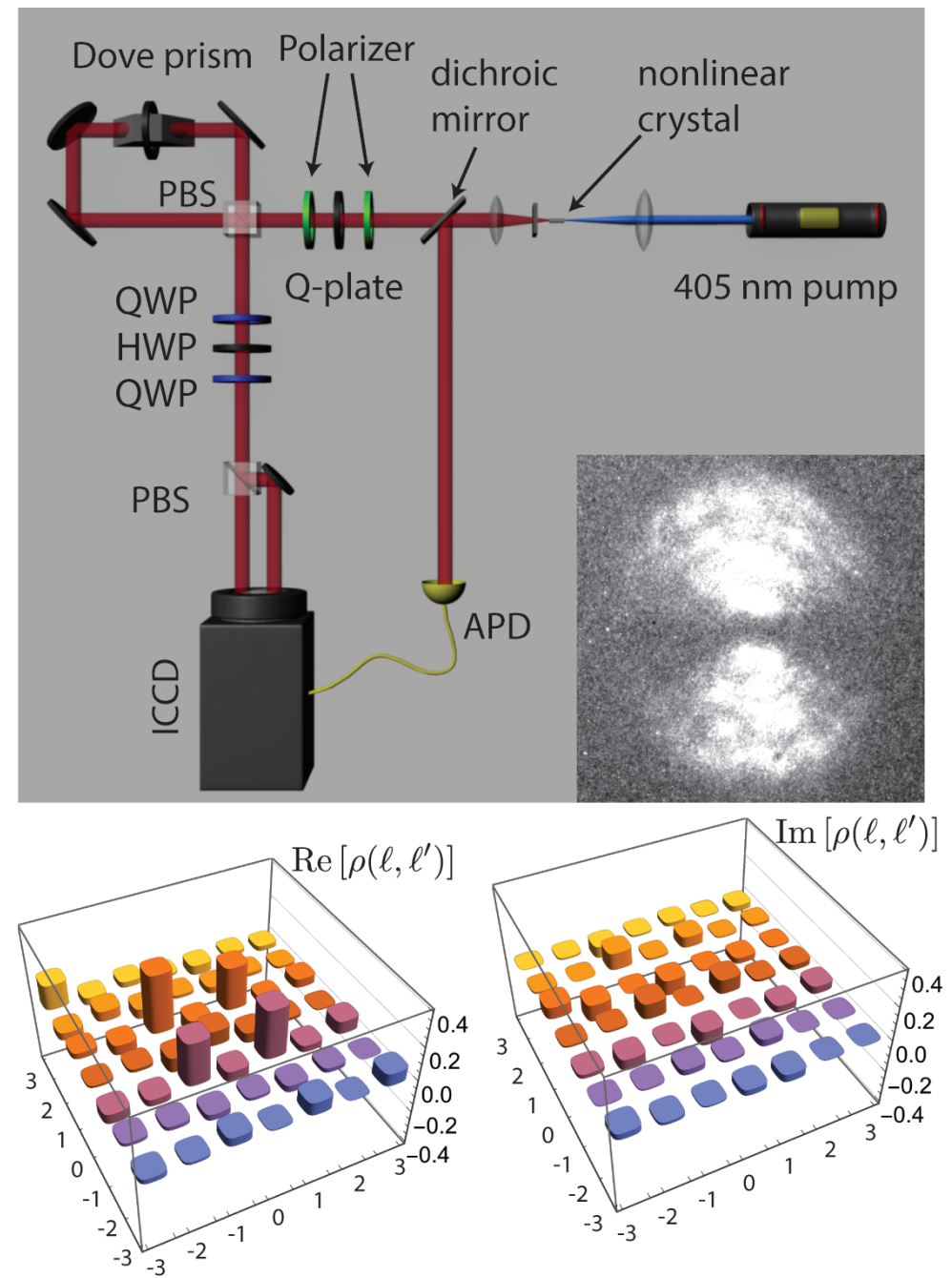

Figure 5.3: Characterization of the transverse structure of single photons. Top left: A PPKTP crystal is pumped with a $405 \mathrm{~nm}$ continuous wave laser beam. Single photons from non-degenerate parametric down-conversion are separated with a dichroic mirror. The idler photons $(830 \mathrm{~nm})$ are detected by an avalanche photo-diode (APD), which heralds the detection of signal photons $(790 \mathrm{~nm})$ with an intensified charge coupled device (ICCD). A q-plate $(\mathrm{q}=1 / 2)$ is placed between two crossed polarizer to prepare an equal superposition of $\ell=1$ and $\ell=-1$ OAM modes. Top right: The transverse structure of single photons captured with an accumulation of 5 -ns-coincidence events over a 1200 sec exposure time. Bottom panel: The real and imaginary parts of the OAM density matrix from experiment.

performed with a classical beam of light (for comparison refer to Fig. 5.1).

We conclude our remarks by analyzing the scaling of our characterization technique. For the full characterization of the density matrix in a Hilbert space 
of dimension $d=2 N+1$, one needs to measure $d^{2}-1$ uknown quantities [117]. The quadratic scaling of the number of required measurement has posed a longstanding challenge for measuring states with large dimensions $[163,8]$. Through the use of a camera for post-selection, we are able to sequence individual images to find $d$ elements of the density matrix simultaneously. This is a crucial practical advantage since our measurement time scales linearly with the dimensionality of the state. Considering the values of exposure times and the resolution of the CCD camera used in this work, we anticipate that our technique can used for characterization of state with a dimensionality, $d$, as large as 100.

\subsection{Summary}

In summary, we have demonstrated a technique for full characterization of the orbital-angular-momentum content of a beam of light. We have achieved this task by directly measuring the elements of the density matrix in the basis of azimuthal angle, and mapping the results to the conjugate basis of OAM via a linear transformation. We have tested our technique by applying it to characterization of both classical beams of light and heralded single photons. Our approach readily scales to very large dimensions, involves no photon loss from post-selection, and is capable of characterizing partially coherent OAM states. To our knowledge, this technique is the only approach that is capable of simultaneously achieving these task. We anticipate that the presented method for characterization of the OAM density matrix will constitute an essential part of quantum information protocols that are based on using the the azimuthal structure of the light field. 


\section{Conclusions}

In this thesis, we have investigated the role of structured light fields as resource for encoding quantum information. The recent progress in the technology of spatial light modulators has enabled precise control of the transverse mode of the optical field. The transverse structure of the light field is in principle an unbounded space, and hence it is an ideal degree of freedom for encoding information. In the first half of this thesis, we have discussed our implementation of a multi-dimensional quantum key distribution system that is based on encoding information on the orbital angular momentum of single photons.

The feasibility of high-dimensional QKD in the spatial domain has been previously demonstrated by encoding information in the transverse linear momentum and position bases. We have shown that the utilization of OAM modes in a long-haul free-space link has the advantage of reducing diffraction-induced loss and cross-talk. Moreover, the inherently discrete nature of orbital angular momentum lends itself to implementation of a generalized version of the BB-84 protocol. Nonetheless, the precise measurement of the OAM of a single photon has been a major roadblock for the implementation of such a protocol. We have overcome this limitation by developing a photon-efficient mode sorting device that can achieve a mode discrimination of greater than $92 \%$. This task has been achieved with advancing the design of a mode-sorter device, originally developed by Miles Padgett's group, through the use of a diffractive beam-splitting element. Chapters two and three of this thesis outline the implementation of our QKD protocol, which is based on a 7-dimensional alphabet encoded in OAM and in the mutually unbiased basis of angular (ANG) modes. In our experimental implementation, we have achieved a mutual information of 2.05 bits per sifted 
photon, which is more than twice the maximum allowable capacity of a twodimensional QKD system. The symbol error rate of our scheme is measured to be $10.5 \%$, which is sufficient for proving security against coherent and individual eavesdropping attacks for an infinite key. While our experiment demonstrates the feasibility of using OAM modes for QKD, several challenges need to be addressed before such a protocol can be employed for practical applications. We have laid out a clear path for how our system can be enhanced to perform practical, high-dimensional QKD using current technology. Professor Boyd's research group is currently pursuing this avenue of research with the ultimate goal of implementing a 10-km long free-space QKD system, with a rate of at least 100 $\mathrm{Mb} / \mathrm{s}$.

While an increased dimensionality is beneficial for a number of the quantum information processing tasks such as QDK, it poses a challenge for the experimentalist who desires to generate and manipulate such states with full control. In the second half of this thesis, we have tackled the problem of characterization of structured light fields. The method of direct measurement, devised by Jeff Lundeen's group, provides a simple means of characterizing large-dimensional states with the aid of complex weak values. We have applied this method to characterization of OAM modes and their superpositions. Through the use of this method, we have been able to measure the probability amplitudes and the relative phase of the OAM components of an arbitrary light field in a 27-dimensional state space. We have then improved the direct measurement technique by combining it with a computational technique known as compressive sensing. Although the direct measurement approach provides an easy-to-implement approach to the problem of state characterization, it does not scale favorably as the dimensionality of the state under investigation increases. We have found that compressive direct measurements (CDMs), can be used to drastically reduce the number of experiments required for the characterization of a wave function. Our experimental results demonstrate an accurate determination of a 192-dimensional state with a fidelity of $90 \%$ using only 25 percent of measurements that are needed for the conventional direct measurement approach. Taking this method to the extremes, we have demonstrated a 350-fold speed up in the process of characterization of 
a 19200-dimensional state.

In the last chapter, we have demonstrated a technique for full characterization of the orbital-angular-momentum content of the light field. This method goes beyond the conventional direct measurement technique by providing the ability to characterize mixed (i.e. incoherent or partially coherent) states while relying exclusively on projective measurements. We have shown that the elements of density matrix in the basis of azimuthal angle can be directly found by registering the interference between a quantum state and its rotated replica. We then convert the measured angular density matrix to basis of orbital angular momentum by using a linear transformation. We have tested our method by applying it to characterization of both classical beams of light and heralded single photons. We have shown that the number of required measurements in this approach scales favorably to large dimensions, while the absence of post-selection loss makes it ideal for characterizing the transverse structure of quantum sources of radiation. We are currently investigating this avenue of research with the ultimate goal of characterizing single-photon and two-photon states, in a Hilbert state spanned by 100 OAM modes. 


\section{Appendix A: Diffraction and radial modes}

It is known that OAM modes with larger mode index $\ell$ have a larger rms (root mean squared) radius in the far field [164]. Given the different radial profiles of different OAM modes, one might be concerned that the security of the protocol is compromised. More specifically, we are interested to know whether Eve can gain information by using the photons that fall outside Bob's aperture? The details of the diffraction behavior depends on the radial profile of the OAM modes as defined in the transmitter's aperture. We consider OAM modes with a top-hat intensity profile such as the ones used in our experiment. Since diffraction plays no role once the Fresnel number is sufficiently large, this issue does not directly apply to our experiment. However, the answer to the question for a low-Fresnelnumber system is not trivial. Below, we show that it is impossible for Eve to gain any information by performing this attack regardless of the Fresnel number of the system.

\section{Intercept and intercept attack}

In an intercept and intercept attack (also known as denial of service) Eve detects a photon without retransmitting it. Since Alice and Bob disregards the frames with no photons in basis reconciliation, Eve will not have any mutual information with the sifted key and hence there is no loss of security. 


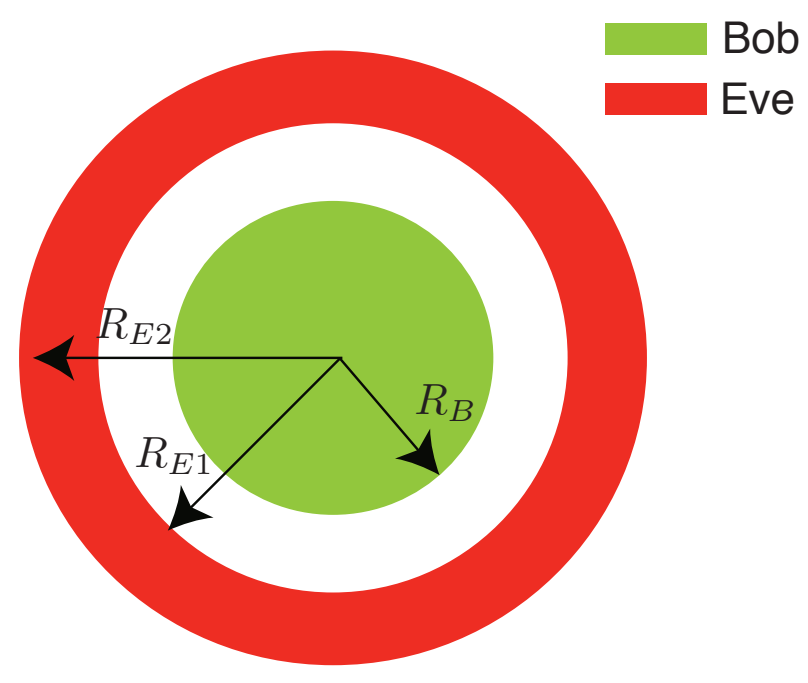

Figure 4: Bob's and Eve's receiving apertures

\section{Intercept and resend attack}

In this type of attack Eve detects a photon that falls outside of Bob's apertures in a random basis. She then resends a photon in a state determined by the result of her measurement into the Bob's aperture. The geometry for Bob's and Eve's apertures are shown in Fig. 4. Note that the $R_{B}, R_{E 1}$ and $R_{E 2}$ are all bound between zero and infinity and so we are considering the most general case.

We demonstrate an OAM mode's optical field at any plane $z$ by $\Psi_{\text {OAM }}^{\ell}(z)$. The probability for detecting each of the OAM modes by Eve can be written as

$$
P_{\mathrm{OAM}}^{\ell}(z)=\int_{R_{E 1}}^{R_{E 2}}\left|\Psi_{\mathrm{OAM}}^{\ell}(z)\right|^{2} r d r .
$$

Assuming Alice chooses to send OAM modes randomly with a probability of $1 / d$ (where $d=2 N+1$ is the total number of modes used), the probability for detecting any OAM mode will be

$$
P_{\mathrm{OAM}}(z)=\frac{1}{d} \sum_{\ell=-N}^{N} \int_{R_{E 1}}^{R_{E 2}}\left|\Psi_{\mathrm{OAM}}^{\ell}(z)\right|^{2} r d r
$$


Similarly, the probability for detecting each of the ANG modes can be written as

$$
P_{\mathrm{ANG}}^{n}(z)=\int_{R_{E 1}}^{R_{E 2}}\left|\Psi_{\mathrm{ANG}}^{n}(z)\right|^{2} r d r,
$$

and the probability for detecting any ANG mode will be

$$
P_{\mathrm{ANG}}(z)=\frac{1}{d} \sum_{n=-N}^{N} \int_{R_{E 1}}^{R_{E 2}}\left|\Psi_{\mathrm{ANG}}^{n}(z)\right|^{2} r d r,
$$

We can simplify Eq. A.4 using the definition of the ANG modes

$$
\Psi_{\mathrm{ANG}}^{n}=\frac{1}{\sqrt{d}} \sum_{\ell=-N}^{N} \Psi_{\mathrm{OAM}}^{\ell} \exp \left(\frac{i 2 \pi n \ell}{d}\right) .
$$

Substituting $\Psi_{\mathrm{ANG}}^{n}$ we get

$$
\begin{aligned}
P_{\mathrm{ANG}}(z) & =\frac{1}{d} \sum_{n=-N}^{N} \int_{R_{E 1}}^{R_{E 2}}\left|\frac{1}{\sqrt{d}} \sum_{\ell=-N}^{N} \Psi_{\mathrm{OAM}}^{\ell} \exp \left(\frac{i 2 \pi n \ell}{d}\right)\right|^{2} r d r \\
& =\frac{1}{d} \int_{R_{E 1}}^{R_{E 2}} \sum_{n=-N}^{N} \sum_{\ell=-N}^{N} \sum_{\ell^{\prime}=-N}^{N} \frac{1}{d} \Psi_{\mathrm{OAM}}^{\ell} \Psi_{\mathrm{OAM}}^{* \ell^{\prime}} \exp \left[\frac{i 2 \pi n\left(\ell-\ell^{\prime}\right)}{d}\right] r d r \\
& =\frac{1}{d} \int_{R_{E 1}}^{R_{E 2}} \sum_{\ell=-N}^{N} \sum_{\ell^{\prime}=-N}^{N} \Psi_{\mathrm{OAM}}^{\ell} \Psi_{\mathrm{OAM}}^{* \ell^{\prime}} \sum_{n=-N}^{N} \frac{1}{d} \exp \left[\frac{i 2 \pi n\left(\ell-\ell^{\prime}\right)}{d}\right] r d r \\
& =\frac{1}{d} \int_{R_{E 1}}^{R_{E 2}} \sum_{\ell=-N}^{N} \sum_{\ell^{\prime}=-N}^{N} \Psi_{\mathrm{OAM}}^{\ell} \Psi_{\mathrm{OAM}}^{* \ell^{\prime}} \delta_{\ell, \ell^{\prime}} r d r \\
& =\frac{1}{d} \int_{R_{E 1}}^{R_{E 2}} \sum_{\ell=-N}^{N}\left|\Psi_{\mathrm{OAM}}^{\ell}\right|^{2} r d r \\
& =P_{\mathrm{OAM}}(z) .
\end{aligned}
$$

The above result shows that it is equally probable for Eve to detect a photon from either the OAM basis or the ANG basis using the beam from any range of radii. Consequently, it is impossible for Eve to gain information by detecting a photon from outside of Bob's aperture. Moreover, the result remains valid at 
any plane $z$ from near field to far field. 


\section{Appendix B: Data for characterization of mixed OAM} states
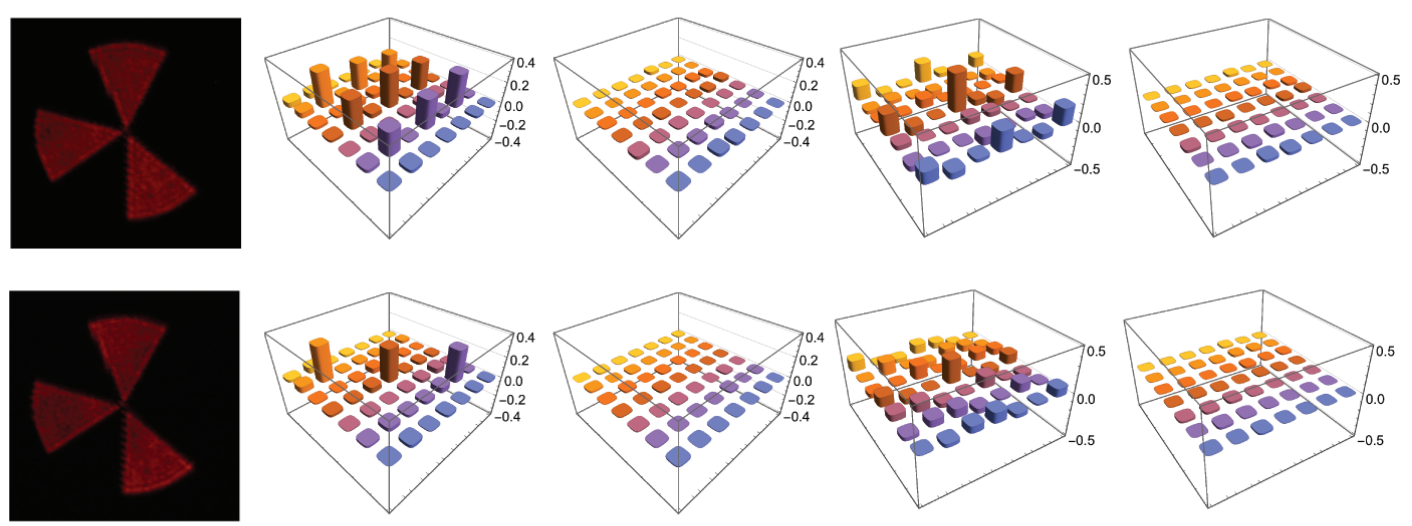

Figure 5: Characterization of a coherent superposition and a mixture of angular wedges. Top panel: Elements of the density matrix for a coherent superposition of three angular wedges $\left(|\Psi\rangle=\left|\Theta_{1}\right\rangle+\left|\Theta_{3}\right\rangle+\left|\Theta_{5}\right\rangle\right)$. Bottom panel: Elements of the density matrix for an incoherent mixture of the same angular wedge states. 

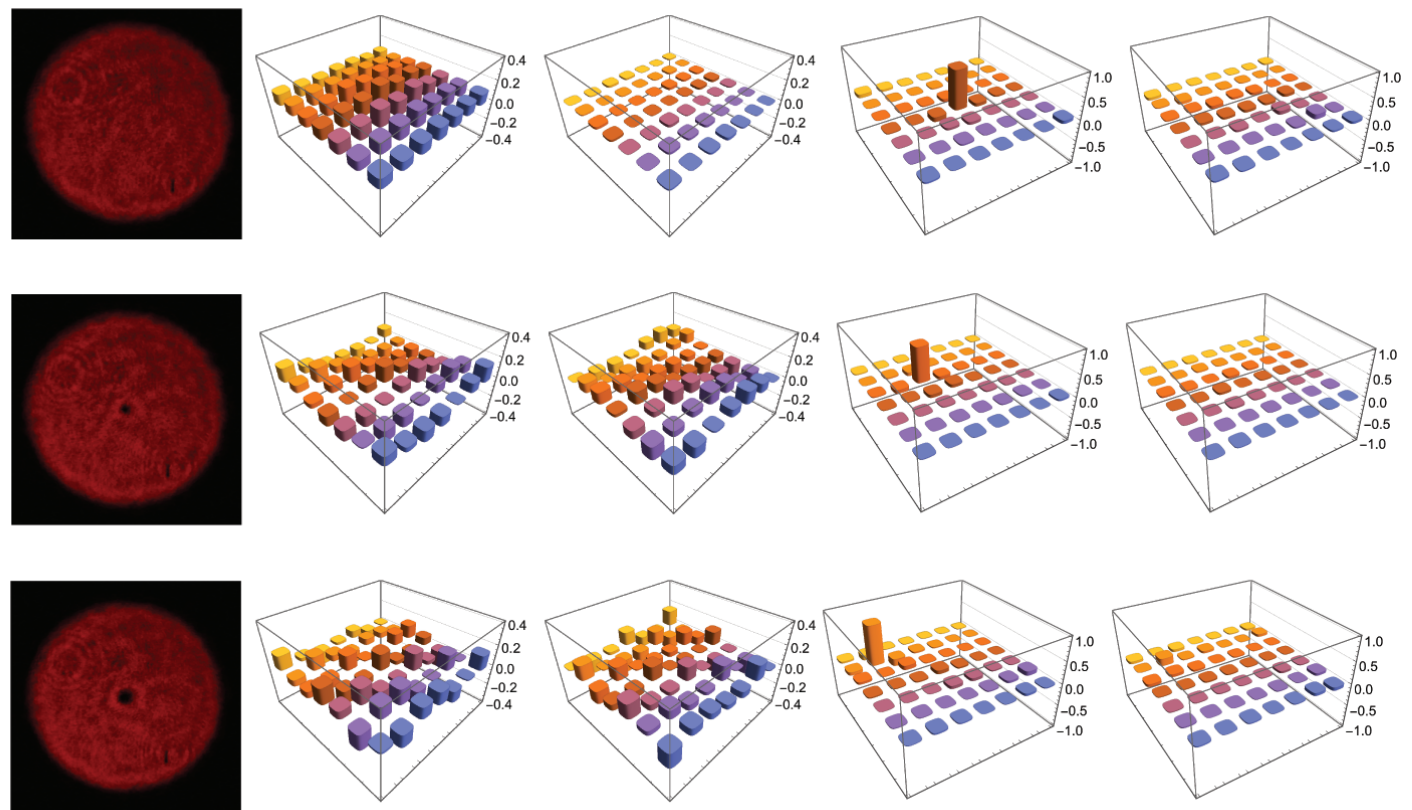

Figure 6: Characterization of OAM eigenstates. Top panel: Elements of the density matrix for $\ell=0$. Middle panel: Elements of the density matrix for $\ell=1$. Bottom panel: Elements of the density matrix for $\ell=2$.
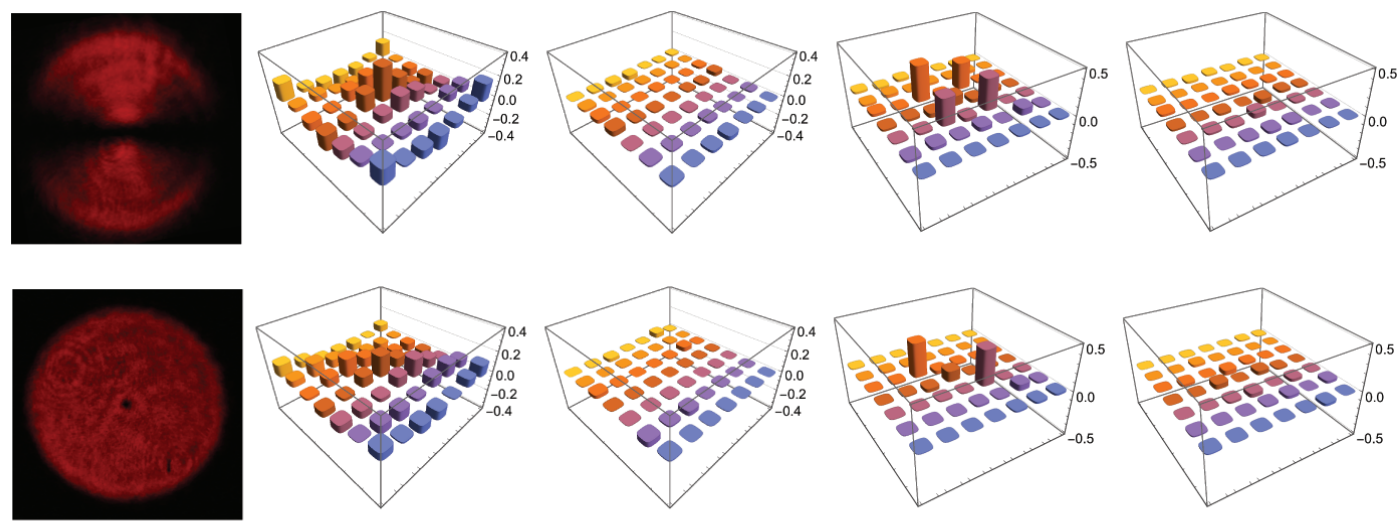

Figure 7: Characterization of a coherent superposition and a mixture of OAM eigenstates. Top panel: Elements of the density matrix for a coherent superposition of two OAM states $(|\Psi\rangle=|\ell\rangle+|-\ell\rangle$, where $\ell=1)$. Bottom panel: Elements of the density matrix for an incoherent mixture of two OAM states $(\rho=|\ell\rangle\langle\ell|+|-\ell\rangle\langle-\ell|$, where $\ell=1)$. 

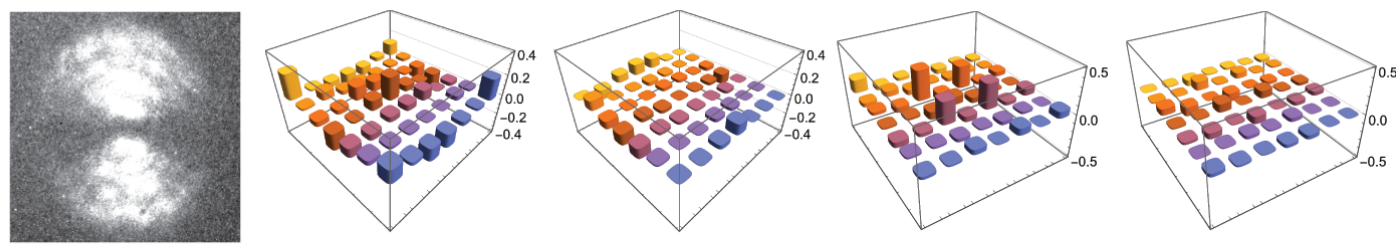

Figure 8: Characterization of a coherent superposition of OAM eigenstates imposed on the transverse structure of single photons. 


\section{Bibliography}

[1] M. Mirhosseini, M. Malik, Z. Shi, and R. W. Boyd, Nat. Commun. 4, 2781 (Nov. 2013).

[2] M. N. O'Sullivan, M. Mirhosseini, M. Malik, and R. W. Boyd, Opt. Express 20(22), 24444 (2012).

[3] G. Berkhout, M. Lavery, J. Courtial, M. Beijersbergen, and M. Padgett, Phys. Rev. Lett. 105(15) (2010).

[4] M. P. J. Lavery, D. J. Robertson, G. C. G. Berkhout, G. D. Love, M. J. Padgett, and J. Courtial, Opt. Express 20(3), 2110 (2012).

[5] M. Mirhosseini, O. S. Magaña-Loaiza, M. N. O'Sullivan, B. Rodenburg, M. Malik, M. P. J. Lavery, M. J. Padgett, D. J. Gauthier, and R. W. Boyd, New J. Phys. 17(3), 033033 (Mar. 2015).

[6] M. Mirhosseini, O. S. Magaña-Loaiza, C. Chen, B. Rodenburg, M. Malik, and R. W. Boyd, Opt. Express 21(25), 30196 (2013).

[7] M. Mirhosseini, B. Rodenburg, M. Malik, and R. W. Boyd, Journal of Modern Optics 61(1), 43 (Feb. 2014).

[8] M. Mirhosseini, O. S. Magaña-Loaiza, S. M. Hashemi Rafsanjani, and R. W. Boyd, Phys. Rev. Lett. 113(9), 090402 (Aug. 2014).

[9] M. Malik, M. Mirhosseini, M. P. J. Lavery, J. Leach, M. J. Padgett, and R. W. Boyd, Nat. Commun. 5, 3115 (Jan. 2014). 
[10] J. S. Lundeen, B. Sutherland, A. Patel, C. Stewart, and C. Bamber, Nature 474(7350), 188 (2011).

[11] J. H. Poynting, Proceedings of the Royal Society A: Mathematical, Physical and Engineering Sciences 82(557), 560 (Jul. 1909).

[12] R. Beth, Phys. Rev. 50(2), 115 (Jul. 1936).

[13] L. Allen, M. Beijersbergen, R. Spreeuw, and J. Woerdman, Phys. Rev. A 45(11), 8185 (1992).

[14] J. D. Jackson, Classical Electrodynamics, 3rd Edition, by John David Jackson, pp. 832. ISBN 0-471-30932-X. Wiley-VCH, July 1998. -1 (Jul. 1998).

[15] L. Mandel and E. Wolf, Cambridge University Press (1995).

[16] (1997).

[17] S. J. Van Enk and G. Nienhuis, Journal of Modern Optics 41(5), 963 (May 1994).

[18] K. Y. Bliokh, M. A. Alonso, E. A. Ostrovskaya, and A. Aiello, Phys. Rev. A 82(6), 063825 (Dec. 2010).

[19] L. Marrucci, C. Manzo, and D. Paparo, Phys. Rev. Lett. 96(16), 163905 (Apr. 2006).

[20] 12(15), 3548 (2004).

[21] Y. Aharonov, D. Albert, and L. Vaidman, Phys. Rev. Lett. 60(14), 1351 (Apr. 1988).

[22] J. S. Lundeen and K. Resch, Phys. Lett. A quant-ph(quant-ph/0501072), 337 (Jan. 2005).

[23] J. Dressel, S. Agarwal, and A. N. Jordan, Phys. Rev. Lett. 104(24), 240401 (2010). 
[24] P. Dixon, D. Starling, A. Jordan, and J. Howell, Phys. Rev. Lett. 102(17) (2009).

[25] N. Ritchie, J. Story, and R. Hulet, Phys. Rev. Lett. 66(9), 1107 (1991).

[26] O. Hosten and P. Kwiat, Science 319(5864), 787 (Feb. 2008).

[27] O. S. Magaña-Loaiza, M. Mirhosseini, B. Rodenburg, and R. W. Boyd, Phys. Rev. Lett. 112(20), 200401 (May 2014).

[28] N. Brunner and C. Simon, Phys. Rev. Lett. 105(1), 010405 (Jul. 2010).

[29] N. Brunner, V. Scarani, M. Wegmüller, M. Legré, and N. Gisin, Phys. Rev. Lett. 93(20), 203902 (Nov. 2004).

[30] A. Feizpour, X. Xing, and A. M. Steinberg, Phys. Rev. Lett. 107(13), 133603 (Sep. 2011).

[31] A. Mair, A. Vaziri, G. Weihs, A. Zeilinger, and Thesis, Nature 412(6844), 313 (Jul. 2001).

[32] J. T. Barreiro, T.-C. Wei, and P. G. Kwiat, Nat Phys. 4(4), 282 (Mar. 2008).

[33] G. Molina-Terriza, J. P. Torres, and L. Torner, Nat Phys. 3(5), 305 (May 2007).

[34] A. M. Yao and M. J. Padgett, Advances in Optics and Photonics 3(2), 161 (2011).

[35] R. Fickler, R. Lapkiewicz, W. N. Plick, M. Krenn, C. Schaeff, S. Ramelow, and A. Zeilinger, Science 338(6107), 640 (Nov. 2012).

[36] J. Wang, J.-Y. Yang, I. M. Fazal, N. Ahmed, Y. Yan, H. Huang, Y. Ren, Y. Yue, S. Dolinar, M. Tur, et al., Nature Photon. 6(7), 488 (Jun. 2012).

[37] N. Cerf, M. Bourennane, A. Karlsson, and N. Gisin, Phys. Rev. Lett. 88(12), 127902 (Mar. 2002). 
[38] M. Bourennane, A. Karlsson, G. Björk, N. Gisin, and N. J. Cerf, J. Phys. A 35(47), 10065 (2002).

[39] G. Gibson, J. Courtial, M. J. Padgett, M. Vasnetsov, V. Pas'ko, S. M. Barnett, and S. Franke-Arnold, Opt. Express 12(22), 5448 (2004).

[40] J. Leach, J. Courtial, K. Skeldon, S. M. Barnett, S. Franke-Arnold, and M. J. Padgett, Phys. Rev. Lett. 92(1) (2004).

[41] O. Bryngdahl, Opt Commun 10(2), 164 (Feb. 1974).

[42] O. Bryngdahl, JOSA 64(8), 1092 (1974).

[43] Y. Saito, S.-i. Komatsu, and H. Ohzu, Opt Commun 47(1), 8 (Aug. 1983).

[44] J. W. Goodman, Introduction to Fourier optics, McGraw-Hill, San Francisco (1968).

[45] L. A. Romero and F. M. Dickey, JOSA A 24(8), 2280 (2007).

[46] D. Prongue, H. P. Herzig, R. Dandliker, and M. T. Gale, Appl. Opt. 31(26), 5706 (1992).

[47] V. Arrizón, U. Ruiz, R. Carrada, and L. A. Gonzalez, JOSA A 24(11), $3500(2007)$.

[48] T. M. Cover and J. A. Thomas, Elements of Information Theory (WileyInterscience, New York, 2006), 2nd ed.

[49] C. H. Bennett and G. Brassard, in Proc. IEEE Int. Conf., Bangalore (1984), pp. 175-179.

[50] N. Gisin, G. Ribordy, W. Tittel, and H. Zbinden, Rev. Mod. Phys. 74(1), 145 (Mar. 2002).

[51] W. K. Wootters and W. H. Zurek, Nature 299(5886), 802 (Oct. 1982).

[52] D. Dieks, Phys. Lett. A 92(6), 271 (Nov. 1982). 
[53] H. Takesue, S. W. Nam, Q. Zhang, R. H. Hadfield, T. Honjo, K. Tamaki, and Y. Yamamoto, Nature Photon. 1(6), 343 (Jun. 2007).

[54] N. Namekata, H. Takesue, T. Honjo, Y. Tokura, and S. Inoue, Opt. Express 19(11), 10632 (May 2011).

[55] A. R. Dixon, Z. L. Yuan, J. F. Dynes, A. W. Sharpe, and A. J. Shields, Opt. Express 16(23), 18790 (Nov. 2008).

[56] H. Bechmann-Pasquinucci and W. Tittel, Phys. Rev. A (2000).

[57] M. Bourennane, A. Karlsson, and G. Björk, Phys. Rev. A (2001).

[58] D. Sych, B. Grishanin, and V. Zadkov, Phys. Rev. A 70(5), 052331 (Nov. 2004).

[59] I. Marcikic, H. de Riedmatten, W. Tittel, H. Zbinden, M. Legré, and N. Gisin, Phys. Rev. Lett. 93(18), 180502 (Oct. 2004).

[60] I. Ali-Khan, C. Broadbent, and J. Howell, Phys. Rev. Lett. 98(6), 060503 (Feb. 2007).

[61] J. Nunn, L. J. Wright, C. Söller, L. Zhang, I. A. Walmsley, and B. J. Smith, Opt. Express 21(13), 15959 (Jul. 2013).

[62] Z. Zhang, J. Mower, D. Englund, F. N. C. Wong, and J. H. Shapiro, Phys. Rev. Lett. 112(12), 120506 (Mar. 2014).

[63] M. Mafu, A. Dudley, S. Goyal, D. Giovannini, M. McLaren, M. J. Padgett, T. Konrad, F. Petruccione, N. Lütkenhaus, and A. Forbes, Phys. Rev. A 88(3), 032305 (2013).

[64] S. Groblacher, T. Jennewein, A. Vaziri, G. Weihs, and A. Zeilinger, New J. Phys. 8(5), 75 (2006).

[65] A. Vaziri, G. Weihs, and A. Zeilinger, Phys. Rev. Lett. 89(24), 240401 (Nov. 2002). 
[66] G. Vallone, V. D'Ambrosio, A. Sponselli, S. Slussarenko, L. Marrucci, F. Sciarrino, and P. Villoresi, arXiv:1402.2932v1 pp. 1-11 (Feb. 2014).

[67] S. Walborn, D. Lemelle, M. Almeida, and P. Ribeiro, Phys. Rev. Lett. 96(9), 090501 (Mar. 2006).

[68] S. Etcheverry, G. Cañas, E. S. Gómez, W. A. T. Nogueira, C. Saavedra, G. B. Xavier, and G. Lima, Sci. Rep. 3:2316 (Jul. 2013).

[69] J. H. Shapiro, Appl. Opt. 13(11), 2614 (1974).

[70] D. A. B. Miller, Appl. Opt. 39(11), 1681 (2000).

[71] D. Slepian, JOSA 55(9), 1110 (1965).

[72] G. A. Tyler, Opt. Lett. 36(23), 4650 (2011).

[73] J. H. Shapiro, Selected Topics in Quantum Electronics, IEEE Journal of 15(6), 1547 (2009).

[74] D. Slepian, Slepian, David Prolate Spheroidal Wave Functions, Fourier Analysis and Uncertainty - IV: Extensions to Many Dimensions; Generalized Prolate Spheroidal Functions (Oct. 1964), http://www.alcatellucent.com/bstj/vol43-1964/articles/bstj43-6-3009.pdf.

[75] B. Rodenburg, Communicating with Transverse Modes of Light, Ph.D. thesis (Oct. 2014).

[76] M. Krenn, R. Fickler, M. Fink, J. Handsteiner, M. Malik, T. Scheidl, R. Ursin, and A. Zeilinger, arXiv:1402.2602 (Feb. 2014).

[77] C. Paterson, Phys. Rev. Lett. 94(15), 153901 (Apr. 2005).

[78] G. A. Tyler and R. W. Boyd, Opt. Lett. 34(2), 142 (2009).

[79] B. Rodenburg, M. Mirhosseini, M. Malik, O. S. Magaña-Loaiza, M. Yanakas, L. Maher, N. K. Steinhoff, G. A. Tyler, and R. W. Boyd, New J. Phys. 16(3), 033020 (Mar. 2014). 
[80] B. Rodenburg, M. Malik, M. O'Sullivan, M. Mirhosseini, and R. Boyd, in Quantum Information and Measurement, Optical Society of America (2012).

[81] R. W. Boyd, B. Rodenburg, M. Mirhosseini, and S. M. Barnett, Opt. Express 19(19), 18310 (2011).

[82] M. Malik, B. Rodenburg, M. Mirhosseini, J. Leach, M. P. J. Lavery, M. J. Padgett, and R. W. Boyd, Opt. Express 20(12), 13195 (2012).

[83] W. K. Wootters and B. D. Fields, Annals of Physics 191(2), 363 (May 1989).

[84] D. Giovannini, J. Romero, J. Leach, A. Dudley, A. Forbes, and M. Padgett, Phys. Rev. Lett. 110(14), 143601 (Apr. 2013).

[85] V. D'Ambrosio, F. Cardano, E. Karimi, E. Nagali, E. Santamato, L. Marrucci, and F. Sciarrino, Sci. Rep. 3:2726 (Sep. 2013).

[86] M. T. Gruneisen, W. A. Miller, R. C. Dymale, and A. M. Sweiti, Appl. Opt. 47(4), A32 (2008).

[87] B. Sun, M. P. Edgar, R. Bowman, L. E. Vittert, S. Welsh, A. Bowman, and M. J. Padgett, Science 340(6134), 844 (May 2013).

[88] P. Zhu, O. Fajardo, J. Shum, Y.-P. Zhang Schärer, and R. W. Friedrich, Nat Protoc 7(7), 1410 (Jun. 2012).

[89] Y.-X. Ren, M. Li, K. Huang, J.-G. Wu, H.-F. Gao, Z.-Q. Wang, and Y.-M. Li, Appl. Opt. 49(10), 1838 (2010).

[90] 14(12), 5588 (2006).

[91] V. Lerner, D. Shwa, Y. Drori, and N. Katz, Opt. Lett. 37(23), 4826 (2012).

[92] B. R. Brown and A. W. Lohmann, IBM Journal of research and Development 13(2), 160 (1969). 
[93] W. H. Lee, Appl. Opt. 18(13), 2152 (1979).

[94] J. P. KIRK and A. L. JONES, JOSA 61(8), 1023 (1971).

[95] D. C. Chu and J. W. Goodman, Appl. Opt. 11(8), 1716 (1972).

[96] J. A. Davis, D. M. Cottrell, J. Campos, M. J. Yzuel, and I. Moreno, Appl. Opt. 38(23), 5004 (1999).

[97] D. Dudley, W. M. Duncan, and J. Slaughter, in H. Urey, ed., Micromachining and Microfabrication (SPIE, 2003), pp. 14-25.

[98] G. Brassard and L. Salvail, EUROCRYPT'93 pp. 410-423 (1994).

[99] C. H. Bennett, G. Brassard, C. Crépeau, and U. M. Maurer, IEEE Trans. Inform. Theory 41(6), 1915 (1995).

[100] I. Csiszar and J. Korner, IEEE Trans. Inform. Theory 24(3), 339 (May 1978).

[101] V. Scarani and R. Renner, Phys. Rev. Lett. 100(20), 200501 (2008).

[102] H. P. Yuen, Quantum Semiclass. Opt. 8(4), 939 (Aug. 1996).

[103] B. Huttner, N. Imoto, N. Gisin, and T. Mor, Phys. Rev. A 51(3), 1863 (Mar. 1995).

[104] N. Lütkenhaus, Phys. Rev. A 61(5), 052304 (Apr. 2000).

[105] G. Brassard, N. Lütkenhaus, T. Mor, and B. Sanders, Phys. Rev. Lett. 85(6), 1330 (Aug. 2000).

[106] M. J. Strain, X. Cai, J. Wang, J. Zhu, D. B. Phillips, L. Chen, M. LopezGarcia, J. L. O'Brien, M. G. Thompson, M. Sorel, et al., Nat. Commun. 5, 4856 (Sep. 2014).

[107] E. Karimi, B. Piccirillo, E. Nagali, L. Marrucci, and E. Santamato, Applied Physics Letters 94(23), 231124 (2009). 
[108] N. Radwell, D. Brickus, T. W. Clark, and S. Franke-Arnold, Opt. Express 22(11), 12845 (Jun. 2014).

[109] V. Scarani, H. Bechmann-Pasquinucci, N. J. Cerf, M. Dusek, N. Lütkenhaus, and others, Rev. Mod. Phys. 81(3), 1301 (2009).

[110] W.-Y. Hwang, Phys. Rev. Lett. 91(5), 057901 (2003).

[111] P. W. Milonni and M. L. Hardies, Phys. Lett. A 92(7), 321 (Nov. 1982).

[112] B. Kanseri, T. Iskhakov, I. Agafonov, M. Chekhova, and G. Leuchs, Phys. Rev. A 85(2), 022126 (2012).

[113] M. Cramer, M. B. Plenio, S. T. Flammia, R. Somma, D. Gross, S. D. Bartlett, O. Landon-Cardinal, D. Poulin, and Y.-K. Liu, Nat. Commun. 1(9), 149 (Dec. 2010).

[114] M. Hofheinz, H. Wang, M. Ansmann, R. C. Bialczak, E. Lucero, M. Neeley, A. D. O'Connell, D. Sank, J. Wenner, J. M. Martinis, et al., Nature 459(7246), 546 (May 2009).

[115] K. Resch, P. Walther, and A. Zeilinger, Phys. Rev. Lett. 94(7), 070402 (Feb. 2005).

[116] M. Beck, C. Dorrer, and I. Walmsley, Phys. Rev. Lett. 87(25), 253601 (Nov. 2001).

[117] D. F. V. James, P. G. Kwiat, W. J. Munro, and A. G. White, Phys. Rev. A 64(5), 052312 (Oct. 2001).

[118] M. G. Raymer, M. Beck, and D. McAlister, Phys. Rev. Lett. 72(8), 1137 (1994).

[119] D. Smithey, M. Beck, M. Raymer, and A. Faridani, Phys. Rev. Lett. 70(9), 1244 (Mar. 1993).

[120] K. Vogel and H. Risken, Phys. Rev. A 40(5), 2847 (Sep. 1989). 
[121] M. Agnew, J. Leach, M. McLaren, F. S. Roux, and R. W. Boyd, Phys. Rev. A 84(6), 062101 (Dec. 2011).

[122] J. Z. Salvail, M. Agnew, A. S. Johnson, E. Bolduc, J. Leach, and R. W. Boyd, Nature Photon. 7(4), 316 (Mar. 2013).

[123] J. Fischbach and M. Freyberger, Phys. Rev. A 86(5), 052110 (Nov. 2012).

[124] S. Wu, Sci. Rep. 3 (2013).

[125] A. Di Lorenzo, Phys. Rev. Lett. 110(1), 010404 (Jan. 2013).

[126] L. Maccone and C. C. Rusconi, Phys. Rev. A 89(2), 022122 (Feb. 2014).

[127] 20(3), 2034 (2012).

[128] G. A. Howland, D. J. Lum, and J. C. Howell, Opt. Express 22(16), 18870 (Aug. 2014).

[129] E. Yao, S. Franke-Arnold, J. Courtial, S. Barnett, and M. Padgett, Opt. Express 14(20), 9071 (Oct. 2006).

[130] B. Jack, M. J. Padgett, and S. Franke-Arnold, New J. Phys. 10(10), 103013 (Oct. 2008).

[131] J. A. Davis, D. E. McNamara, D. M. Cottrell, and T. Sonehara, Opt. Lett. 39(10), 1549 (2000).

[132] S. M. Barnett and D. T. Pegg, Phys. Rev. A 41(7), 3427 (1990).

[133] A. Shabani, R. L. Kosut, M. Mohseni, H. Rabitz, M. A. Broome, M. P. Almeida, A. Fedrizzi, and A. G. White, Phys. Rev. Lett. 106(10), 100401 (Mar. 2011).

[134] G. Howland and J. Howell, Phys. Rev. X 3(1), 011013 (Feb. 2013).

[135] W.-T. Liu, T. Zhang, J.-Y. Liu, P.-X. Chen, and J.-M. Yuan, Phys. Rev. Lett. 108(17), 170403 (Apr. 2012). 
[136] D. Gross, Y.-K. Liu, S. T. Flammia, S. Becker, and J. Eisert, Phys. Rev. Lett. 105(15), 150401 (Oct. 2010).

[137] O. Katz, Y. Bromberg, and Y. Silberberg, Appl. Phys. Lett. 95(13), 131110 (2009).

[138] R. G. Baraniuk, IEEE Signal Process. Mag. (2008).

[139] R. Jozsa, Phys. Rev. A 76(4), 044103 (Oct. 2007).

[140] J. Romberg, IEEE Signal Process. Mag. 25(2), 14 (2008).

[141] S. L. Campbell and C. D. Meyer, Generalized inverses of linear transformations, vol. 56 (SIAM, 2009).

[142] E. Candes and J. Romberg, Inverse problems 23(3), 969 (2007).

[143] P. Zerom, K. W. C. Chan, J. C. Howell, and R. W. Boyd, Phys. Rev. A 84(6), 061804 (Dec. 2011).

[144] O. S. Magaña-Loaiza, G. A. Howland, M. Malik, J. C. Howell, and R. W. Boyd, Appl. Phys. Lett. 102(23), 231104 (Jun. 2013).

[145] M. Mirhosseini, M. Malik, M. Lavery, J. Leach, M. Padgett, and R. W. Boyd, Frontiers in Optics FW4A.3 (Oct. 2012).

[146] I. Moreno, J. L. Martinez, and J. A. Davis, Appl. Opt. 46(6), 881 (2007).

[147] C. Li, W. Yin, and Y. Zhang, in CAAM report (Apr. 2009).

[148] 20(3), 2034 (2012).

[149] G. A. Howland, J. Schneeloch, D. J. Lum, and J. C. Howell, Phys. Rev. Lett. 112, 253602 (2014).

[150] R. H. Hadfield, Nature Photon. 3(12), 696 (2009).

[151] W. L. Chan, M. L. Moravec, R. G. Baraniuk, and D. M. Mittleman, Opt. Lett. 33(9), 974 (2008). 
[152] J. R. Fienup, Appl. Opt. 21(15), 2758 (1982).

[153] R. Blume-Kohout, New J. Phys. 12(4), 043034 (Apr. 2010).

[154] E. Wigner, Phys. Rev. 40(5), 749 (Jun. 1932).

[155] (Jun. 2015), 1506.08892.

[156] C. Ferrie and J. Combes, Phys. Rev. Lett. 112(4), 040406 (Jan. 2014).

[157] A. N. Jordan, J. Martinez-Rincon, and J. C. Howell, Phys. Rev. X 4(1), 011031 (Mar. 2014).

[158] G. C. Knee and E. M. Gauger, Phys. Rev. X 4(1), 011032 (Mar. 2014).

[159] H. Qassim, F. M. Miatto, J. P. Torres, M. J. Padgett, E. Karimi, and R. W. Boyd, JOSA B 31(6), A20 (Jun. 2014).

[160] R. T. Thew, A. G. White, and W. J. Munro, Phys. Rev. A 66(1), 012303 (Jul. 2002).

[161] F. Steinlechner, P. Trojek, M. Jofre, H. Weier, D. Perez, T. Jennewein, R. Ursin, J. Rarity, M. W. Mitchell, J. P. Torres, et al., Opt. Express 20(9), 9640 (Apr. 2012).

[162] R. Fickler, M. Krenn, R. Lapkiewicz, S. Ramelow, and A. Zeilinger, Sci. Rep. 3 (May 2013).

[163] M. Agnew, J. Leach, M. McLaren, F. S. Roux, and R. W. Boyd, Phys. Rev. A 84(6) (2011).

[164] R. L. Phillips and L. C. Andrews, Appl. Opt. 22(5), 643 (1983). 